UNITED STATES DEPARTMENT OF THE INTERIOR GEOLOGICAI SURVEY
JUN 291970

EIV. MINES \& GEOLOGY

\title{
ANALYSES OF ROCK AND STREAM-SEDIMENT SAMPLES FROM THE \\ CRAIG B-5 QUADRANGLE, ALASKA
}

By

Allen L. Clark, Henry C. Berg, Donald A. Grybeck,

A. Thomas Ovenshine, and Raymond Wehr

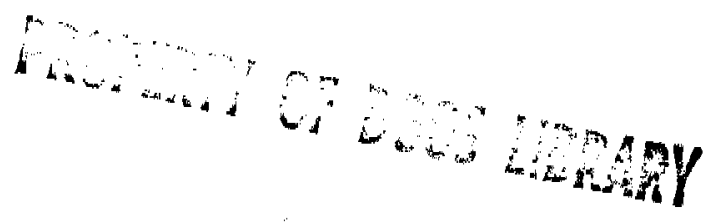

Open-file report

1970

This report is preliminary and has not been edited or reviewed for conformity with Geological Survey standards 


\section{ANALYSES OF ROCK AND STREAM-SEDIMENT SAMPLES FROM THE}

CRAIG B-5 QUADRANGLE, ALASKA

By

Allen L. Clark, Henry C. Berg, Donald A. Grybeck, A. Thomas Ovenshine, and Raymond Wehr

\section{INTRODUCTION}

Analytical data for 58 rock and 41 stream-sediment samples from the Craig B-5, 1:63,360-scale quadrangle are presented in this report, together with a statistical treatment of the data. The samples were collected in 1969 as part of the Heavy Metals Program of the U.S. Geological Survey.

The most comprehensive discussion of the geology of the study area is a report by A. F. Buddington and Theodore Chapin (1929). Known metalliferous lodes of the area are descrlbed and additional references to specific areas are given by Berg and Cobb (1967). Additional data is given in reports by Condon (1961), Smith (1914), and Wright and Wright (1908). Supplemental publications are being prepared on the general geology and mineral occurrences of the study area.

\section{Procedures and treatment of data}

Standard procedures were followed in the collection and preparation of samples.

Rock samples are primarily grab samples from mineral occurrences and outcrops. They were chosen for analysis to provide data on background, because they were in the area of mineral occurrences or streamsediment anomalies, because they were strongly iron stained, or contained visible sulfides.

Stream-sediment samples were generally collected from the active stream channel; where this was not possible, samples were collected from bank or terrace deposits adjacent to the channel.

Rock samples were crushed and pulverized and the minus 80 mesh fraction analyzed. Stream-sediment samples were dried, sieved, and the minus 80 mesh fraction analyzed. The minus 80 mesh fractions of the 
samples were analyzed for 30 elements by the six-step semiquantitative spectrographic method and for gold by the atomic absorption method.1/

The spectrographic analyses were reported in percentage (pct) or parts per million (ppm) to the nearest number in the series $1.0,0.7$, $0.5,0.3,0.2,0.15,0.1$, etc. The precision of a reported value is approximately plus 100 percent or minus 50 percent. Analyses for gold by the atomic absorption method are accurate to $t 100$ percent. Minimum limits of determination for each element are given on page 5 . Semiquantitative spectrographic analyses were done by $\mathrm{K}$. J. Curry and atomic absorption analyses were done by R. L. Miller, R. B. Tripp, H. D. King, and A. L. Meier.

Locations of the rock and stream-sediment samples are shown on Plate 1. Rock sample descriptions are given in table 1 and rock sample analyses are tabulated in table 2 and stream-sediment analyses are tabulated in table 3 .

The results of the analyses of the rock and strean-sediment analyses have been processed by means of a computer program known as GEOSUM and are presented in tables 2 and 3 . The GEOSUM program is designed primarily for summarizing and tabulating geochemical data--especially data from semiquantitative spectrographic analyses (commonly referred to as six-step spectrographic analyses) by the laboratories of the U.S. Geological Survey.

The program output consists of: (a) a tabulation of the data, (b) histograms and cumulative frequency distributions for all elements except tungsten, and (c) a statistical summary which includes geometric means and geometric deviations.

$\underline{1}$ Analyses for 29 elements by semiquantitative analyses and for gold by atomic absorption are given in the tables. Semiquantitative analyses for gold are omitted. 
Table 1.--Description of rock, vein, and altered zone samples. (All samples are of representative material.) Sample localities are shown by sample number plotted on the accompanying map, Plate 1.

Sample No. Lab. No.

\section{Sample Description}

\begin{tabular}{r|r}
1 & ACL-320 \\
2 & -325 \\
3 & -326 \\
3 & -327 \\
3 & -328 \\
4 & -329 \\
5 & -330 \\
5 & -331 \\
5 & -332 \\
5 & -333 \\
5 & -334 \\
6 & -335 \\
7 & ACE-651 \\
7 & -652 \\
8 & ACL -323 \\
8 & -324 \\
9 & -347 \\
10 & -345 \\
11 & -346 \\
12 & -322 \\
13 & ACE-949 \\
13 & -950 \\
14 & ACL-458 \\
15 & -457 \\
16 & -456 \\
17 & -454 \\
17 & -455 \\
18 & -453 \\
19 & -452 \\
20 & -460 \\
20 & -461 \\
21 & -462 \\
22 & -463 \\
22 & -464 \\
22 & -465 \\
23 & -466 \\
24 & -474 \\
24 & -475 \\
24 & -476 \\
24 &
\end{tabular}

S1llceous graywacke

Argillite with minor pyrite

Quartz vein

Argillite

Quartz-chalcopyrite pod

Argilite

Pyritized argil1ite

Pyritized argilite

Pyritized argillite

Pyritized argillite

Pyritized argil1te

Pyritized argilite

Quartz diorite with pyrite

Hornfels with pyrite

Monzonite with pyrite

Monzonite with pyrite

Quartz diorite

Quartz diorite

Andesite

Monzonite

Volcanic breccia

Volcanic breccla

Argillite with pyrite

Argillite

Argillite with quartz velns

Argillite with pyrite quartz vein

Argillite with pyrite quartz vein

Quartz velned argillite

Limestone

Quartz monzonite

Quartz veln

Iron-stained quartz monzonite

Aplite dike

Greenstone

Quartz monzonite

Quartz monzonite

Altered granite

Tactite

Stllcified grantte

Hornfelsed arg111ite 
Table 1.--Description of rock, vein, and altered zone samples. (A11 samples are of representative materlal.) Sample localities are shown by sample number plotted on the accompanying map, Plate 1 .--Continued

\begin{tabular}{crl} 
Sample No. & Lab. No. & \multicolumn{1}{c}{ Sample Description } \\
\hline 25 & ACL-484 & Quartz pyrite veinlet \\
26 & -568 & Iron-stained argillite \\
26 & -569 & Iron-stained argillite \\
26 & -570 & Limonite \\
26 & -571 & Iron-stained arg1lilte \\
26 & -572 & Altered monzonite \\
27 & -617 & Pyrrhotite rich quartzite \\
27 & -618 & Aplite dike \\
27 & -619 & Quartzite with pyrite-pyrrhotite and chalcopyrite \\
27 & -573 & Aplite dike \\
28 & -574 & Pyritized black argillite \\
29 & -620 & Pyrite argilitte \\
30 & -575 & Aplite dike \\
30 & -576 & Quartz vein with gulfides \\
30 & -577 & Iron-stained argililte \\
31 & -621 & Phyllite with pyrite and minor chalcopyrite \\
32 & -485 & Tactite with pyrrhotite \\
32 & -486 & Pyrrhotite and pyrite
\end{tabular}


Explanation of Tables. 2 and 3

Analytical results from rock and stream-sediment samples are given in Tables 2 and 3 as analytical values such as $7.0000 \mathrm{ppm}, 10.0000$ percent, etc., or as qualified values expressed as a letter. These letter codes are $N=$ not detected, $L *$ less than specifled limit of detection, $G=$ greater than value shown, $B=$ no data, $H=$ interference. The term $T$ - trace, but does not occur in these data. Note that the right-most zero. digits for each analytical value may or may not be significant. The specified limits of detection are as follows:

\section{Specified limits of detection}

$\begin{array}{lccccc}\text { FE PCT } & \text { MG PCT } & \text { CA PCT } & \text { TI PCT } & \text { MN PPM } & \text { AG PPM } \\ 0.05000 & 0.02000 & 0.05000 & 0.00200 & 20.00000 & 0.10000 \\ \text { AS PPM } & \text { AU PPM } & \text { B PPM } & \text { BA PPM } & \text { BE PPM } & \text { BI PPM } \\ 0.20000 & 0.02000 & 10.00000 & 20.00000 & 1.00000 & 10.00000 \\ \text { CO PPM } & \text { CR PPM } & \text {. CU PPM } & \text { LA PPM } & \text { MO PPM } & \text { NB PPM } \\ 5.00000 & 5.00000 & 2.00000 & 20.00000 & 2.00000 & 10.00000 \\ \text { NI PPM } & \text { PB PPM } & \text { SB PPM } & \text { SC PPM } & \text { SN PPM } & \text { SR PPM } \\ 2.00000 & 10.00000 & 0.50000 & 5.00000 & 10.00000 & 50.00000 \\ \text { V PPM } & \text { W PPM } & \text { Y PPM } & \text { ZN PPM } & \text { ZR PPM } & \\ 5.00000 & 50.00000 & 5.00000 & 25.00000 & 10.00000 & \end{array}$

Semiquantitative spectrographic analyses by the U.S. Geologica1 Survey are reported as geometric midpoints $(1.0,0.7,0.5,0.3,0.2$, $0.15,0.1$, etc.) of geometric backets having the boundaries $1.2,0.83$, $0.56,0.38,0.26,0.18,0.12,0.083$, etc. The frequency distributions and histograms are on logarithmic scales and are computed using these brackets as class intervals, for example:

\begin{tabular}{ccc} 
Reported value (ppm) & \multicolumn{2}{c}{ Limits } \\
1.0 & .83 & 1.2 \\
1.5 & 1.2 & 1.8 \\
2.0 & 1.8 & 2.6 \\
3.0 & 2.6 & 3.8 \\
5.0 & 3.8 & 5.6 \\
7.0 & 5.6 & 8.3 \\
100 & 8.3 & 12.0
\end{tabular}


On the histograms decimal numbers are shown as powers of 10 , for example:

$$
\begin{aligned}
& 7.0 \mathrm{E}-01 \text { means } 7.0 \times 10^{-1} \text { or } 0.7 \\
& 7 . \mathrm{OE} 00 \text { means } 7.0 \times 10^{0} \text { or } 7.0 \\
& 7 . \mathrm{OE} 01 \text { means } 7.0 \times 10^{1} \text { or } 70.0 \\
& 7 . \mathrm{OE} 02 \text { means } 7.0 \times 10^{2} \text { or } 700.0 \\
& 7 . \mathrm{OE} 03 \text { means } 7.0 \times 10^{3} \text { or } 7,000.0
\end{aligned}
$$

The histograms are constructed of $X^{\prime} s$, each of which represents 1 percent of the total number (309) of samples.

The histograms and the statistics given below them are derived only from data values within the ranges of analytical determination ("analytical values"). The histograms are, therefore, incomplete, and the statistics are biased if data values qualified with $\mathrm{N}, \mathrm{L}, \mathrm{C}, \mathrm{T}$, or $H$ codes are present. (See the histogram and statistics below it for tin, which are calculated from only one sample.) Statistical estimates that are unbiased in this regard are given at the end of Table 1 . The geometric means is the antilogarithm of the arithmetic mean of the logs of the analyses and an estimate of "central tendency," or of a characteristic value; , of a frequency distribution that is approximately symmetrical on a $\log$ scale, and is therefore useful for characterizing many geochemical distributions. The geometric mean is not an estimate of geochemical abundance. The geometric deviation is the antilogarithm of the standard deviation of the logs of the analyses. See USGS Professlonal Paper 574-B for further discussion and USGS Bulletin 1147E, p. 20-23, for further discussion and explanation of geometric deviation.

In the computations performed to produce the statistical summary at the end of Tables 2 and 3 , all elements are ignored where one or more of the unqualified data values is less than the analytical limit of detection specified on input or where any data values are qualified with the $G$ (greater than) code. Data values qualified with $B$ or $H$ are not used in the computations. Where none of the data values for an element are qualified the mean and deviation should be the same as those given in the preceding section. Where data are qualified with the codes $\mathrm{N}, \mathrm{L}$, or $T$, the estimates of geometric mean and deviation are based on a method by A. J. Cohen for treating censored distributions. The application of this method of geochemical problems is described in USGS Professiona1 Paper 574-B. The estimates are unbiased in a strict sense only where the data are derived from a lognormal parent population, but experiments have shown that large departures from this requirement may not greatly invalidate the results. Acceptance and use of the estimates, however, is the responsibility of the Individual. 
Berg, H. C., and Cobb, E. H., 1967, Metalliferous lode deposits of Alaska: U.S. Geol. Survey Bull. 1246, p. 164-184.

Buddington, A. F., and Chapin, Theodore, 1929, Geology and mineral deposits of southeastern Alaska: U.S. Geol. Survey Bull. 800, 398 p.

Condon, W. H., 1961, Geology of the Craig quadrangle, Alaska: U.S. Geol. Survey Bull. 1108-B, 43 p.

Miesch, A. T., 1963, Distribution of elements in Colorado Plateau uranium deposits--A preliminary report: U.S. Geol. Survey Bull. 1147-E, $57 \mathrm{p}$.

1967, Methods of computation for estimating geochemical abundance: U.S. Geol. Survey Prof. Paper 574-B, 15 p.

Smith. P. S., 1914, Lode mining in the Ketchikan region, Alaska In Mineral resources of Alaska: U.S. Geo1. Survey Bull. 592, p. 75-84.

Wright, F. E., and Wright, C. W., 1908, The Ketchikan and Wrangell mining districts, Alaska: U.S. Geol. Survey Bul1. 347, 210 p. 
TABLE 2. CRAIG B-5 ROCK SAMPLES

\begin{tabular}{|c|c|c|c|c|c|c|c|c|c|c|c|c|c|c|c|}
\hline & SAMPLE & FE PCT & MG PCI & CA PCT & TI PCT & MN PQM & $\triangle G P$ & $P M$ & 45 & PPM & $A U$ & PPM & B PF & & BA PPM \\
\hline 1 & $A C L 320$ & 2.0000 & 1.0000 & 0.7000 & 0.1500 & 300.0000 & 0.0 & $N$ & 0.0 & $\mathbf{N}$ & 0.0 & $\mathrm{~N}$ & 30.0000 & & 700.0000 \\
\hline 2 & ACL 325 & 3.0000 & 1.5000 & 0.7000 & 0.3000 & 1500.0000 & 0.0 & N & 0.0 & $\mathbf{N}$ & 0.0 & $\mathbf{N}$ & 30.0000 & & 150.0000 \\
\hline 3 & $A C L 326$ & 3.0000 & 0.7000 & 0.7000 & 0.2000 & 500.0000 & 0.0 & $\mathbf{N}$ & 0.0 & $\mathbf{N}$ & 0.0 & $\mathbf{N}$ & 0.0 & t & 300.0000 \\
\hline 3 & $A C L 327$ & 10.0000 & 3.0000 & 0.7000 & 0.7000 & 1500.0000 & 0.0 & $\mathbf{N}$ & 0.0 & $\mathbf{N}$ & 0.0 & $\mathbf{N}$ & 30.0000 & & 700.0000 \\
\hline 3 & $A C L 328$ & 15.0000 & 2.0000 & 1.0000 & 0.7000 & 1500.0000 & 0.500 & & 0.0 & $\mathbf{N}$ & 0.0 & $\mathbf{N}$ & 50.0000 & & 200.0000 \\
\hline 4 & ACL 324 & 7. 0000 & 3.0000 & 5.0000 & 0.7000 & 1000.0000 & 0.0 & $\mathbf{N}$ & 0.0 & $\mathbf{N}$ & 0.0 & $\mathbf{N}$ & 0.0 & $\mathbf{L}$ & 300.0000 \\
\hline 5 & $A C L 330$ & 5.0000 & 0.7000 & 2.0000 & 0.5000 & 500.0000 & 0.0 & $\mathbf{t}$ & 0.0 & $\mathbf{N}$ & 0.0 & $\mathbf{N}$ & 20.0000 & & 700.0000 \\
\hline 5 & $A C L 331$ & 7.0000 & 1.0000 & 1.5000 & 0.7000 & 700.0000 & 0.0 & $\mathbf{N}$ & 0.0 & $\mathbf{N}$ & 0.0 & $\mathbf{N}$ & 15.0000 & & 300.0000 \\
\hline 5 & $\mathrm{ACL} 332$ & 3.0000 & 1.0000 & 3.0000 & 0.3000 & 700.0000 & 0.0 & $\mathbf{N}$ & 0.0 & $N$ & 0.0 & $\mathbf{N}$ & 0.0 & $\mathbf{N}$ & 300.0000 \\
\hline 5 & $A C L 333$ & 3.0000 & 1.5000 & 2.0000 & 0.2000 & 500.0000 & 0.0 & $N$ & 0.0 & $\mathbf{N}$ & 0.0 & $\mathbf{N}$ & 0.0 & $\mathbf{N}$ & 200.0000 \\
\hline 5 & $\mathrm{ACL} 334$ & 3.0000 & 1.5000 & 0.7000 & 0.3000 & 700.0000 & 0.0 & $\mathrm{~N}$ & 0.0 & $\mathbf{N}$ & 0.0 & $\mathbf{N}$ & 30.0000 & & 700.0000 \\
\hline 6 & ACL 335 & 3.0000 & 0.7000 & 1.0000 & 0.1500 & 1500.0000 & 0.0 & $N$ & 0.0 & $\mathbf{N}$ & 0.0 & $\mathbf{N}$ & 0.0 & $N$ & 300.0000 \\
\hline 7 & ACE651 & 7.0000 & 3.0000 & 5.0000 & 0.7000 & 1000.0000 & 0.0 & N & 0.0 & N & 0.0 & $\mathbf{N}$ & 0.0 & L & 150.0000 \\
\hline 7 & ACE 652 & 15.0000 & 3.0000 & 3.0000 & 0.7000 & 1500.0000 & 0.0 & $N$ & 0.0 & $\mathbf{N}$ & 0.0 & $\mathbf{N}$ & 0.0 & $\mathbf{L}$ & 300.0000 \\
\hline 8 & $A C L 323$ & 3.0000 & 1.5000 & 3.0000 & 0.3000 & 700.0000 & 0.0 & $\mathbf{L}$ & 0.0 & N & 0.0 & N & 0.0 & $\mathbf{N}$ & 150.0000 \\
\hline 8 & $A C L 324$ & 3.0000 & 1.5000 & 3.0000 & 0.3000 & 500.0000 & 0.0 & $\mathbf{N}$ & 0.0 & N & 0.0 & $\mathbf{N}$ & 0.0 & $\mathbf{N}$ & 300.0000 \\
\hline 9 & $A C L 347$ & 15.0000 & 2.0000 & 5.0000 & 0.3000 & 1500.0000 & 0.0 & $\mathbf{N}$ & 0.0 & $\mathbf{N}$ & 0.0 & $\mathbf{N}$ & 0.0 & $\mathbf{N}$ & 300.0000 \\
\hline to & $\mathrm{ACL} 345$ & 5.0000 & 2.0000 & 5.0000 & 0.3000 & 500.0000 & 0.0 & $\mathbf{N}$ & 0.0 & $\mathbf{N}$ & 0.0 & $\mathbf{N}$ & 0.0 & $\mathbf{N}$ & 150.0000 \\
\hline 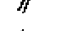 & ACL 346 & 10.0000 & 5.0000 & 5.0000 & 0.7000 & 1500.0000 & 0.0 & $\mathbf{N}$ & 0.0 & $\mathbf{N}$ & 0.0 & $\mathbf{N}$ & 0.0 & $\mathbf{L}$ & 300.0000 \\
\hline 12 & $\mathrm{ACL} 322$ & 1.5000 & 0.7000 & 2.0000 & 0.1500 & 500.0000 & 0.0 & N & 0.0 & $\mathbf{N}$ & 0.0 & $\mathbf{N}$ & 0.0 & $\mathbf{N}$ & 150.0000 \\
\hline$B$ & ACE 949 & $\$ 5.0000$ & 3.0000 & 5.0000 & 0.5000 & 1500.0000 & 0.0 & N & 0.0 & $N$ & 0.0 & $\mathbf{N}$ & 70.0006 & & $0.0 \quad 1$ \\
\hline 13 & ACE950 & 10.0000 & 3.0000 & 7.0000 & 0.5000 & 1000.0000 & 0.0 & N & 0.0 & $\mathbf{N}$ & 0.0 & $\mathbf{N}$ & 0.0 & $N$ & 70.0000 \\
\hline 14 & $A C L 458$ & 3.0000 & 0.7000 & 0.1500 & 0.5000 & 100.0000 & 0.0 & $L$ & 0.0 & $\mathbf{N}$ & 0.0 & $\mathbf{N}$ & 70.0000 & & 5000.0000 \\
\hline 15 & $A C L 457$ & 5.0000 & 1.5000 & 0.7000 & 0.7000 & 300.0000 & 0.0 & L & 0.0 & N & 0.0 & $N$ & 30.0000 & & 300.0000 \\
\hline 16 & ACE 456 & $0.0 \quad 1$ & 1.0000 & $20.0000 \mathrm{G}$ & 0.0300 & 70.0000 & 0.0 & $\mathbf{N}$ & 0.0 & $\mathbf{N}$ & 0.0 & $N$ & 0.0 & $\mathbf{N}$ & $0.0 \quad L$ \\
\hline 17 & ACL 454 & 3.0000 & 2.5000 & 0.7000 & 0.1000 & 300.0000 & 0.0 & N & 0.0 & $\mathbf{N}$ & 0.0 & $\mathbf{N}$ & 70.0000 & & 2000.0000 \\
\hline$Q^{17}$ & ACE 455 & 15.0000 & 7.0000 & 7.0000 & 1.0000 & 1500.0000 & 0.700 & & 0.0 & $N$ & 0.0 & $\mathbf{N}$ & 0.0 & $\mathbf{L}$ & 700.0000 \\
\hline $\mathbf{N}_{1 / 8}$ & $A C L 453$ & 7.0000 & 2.0000 & 3.0000 & 0.5000 & 1500.0000 & 0.0 & $\mathbf{N}$ & 0.0 & $\mathbf{N}$ & 0.0 & $\mathbf{N}$ & 30.0008 & & 1500.0000 \\
\hline 19 & $A C L 452$ & $0.0 \quad \mathrm{~L}$ & 0.7000 & 20.00006 & 0.0300 & 70.0000 & 0.0 & $\mathbf{N}$ & 0.0 & $N$ & 0.0 & $M$ & 0.0 & $\mathbf{N}$ & 150.0000 \\
\hline 20 & ACL460 & 3.0000 & 0.7000 & 1.5000 & 0.3000 & 1000.0000 & 0.0 & $N$ & 0.0 & N & 0.0 & $\mathbf{N}$ & 0.0 & N & 300.0000 \\
\hline 20 & ACL461 & 0.5000 & 0.0700 & 0.7000 & 0.0700 & 100.0000 & 0.0 & N & 0.0 & $\mathbf{N}$ & 0.0 & $\mathbf{N}$ & 0.0 & $\mathbf{N}$ & 100.0000 \\
\hline $2 t$ & ACL 462 & 1.5000 & 0.5000 & 1.5000 & 0.1500 & 500.0000 & 0.0 & $\mathbf{N}$ & 0.0 & N & 0.0 & $\mathbf{N}$ & 0.0 & $\mathbf{N}$ & 200.0000 \\
\hline 22 & $A C L 463$ & 0.7000 & 0.1500 & 1.0000 & 0.0500 & 150.0000 & 0.0 & $\mathbf{N}$ & 0.0 & N & 0.0 & $\mathbf{N}$ & 0.0 & $\mathbf{L}$ & 300.0000 \\
\hline 22 & $A C L 464$ & 20.0000 & 0.7000 & 15.0000 & 0.1000 & 5000.00006 & 0.0 & N & 0.0 & N & 0.0 & $\mathbf{N}$ & 15.0000 & & $0.0 \quad \mathrm{~L}$ \\
\hline 22 & $A C L 465$ & 1.5000 & 0.7000 & 2.0000 & 0.1500 & $1000 \cdot 0000$ & 0.0 & N & 0.0 & $\mathbf{N}$ & 0.0 & $\mathbf{N}$ & 0.0 & $\mathbf{N}$ & 150.0000 \\
\hline 23 & ACL 466 & 1.5000 & 0.3000 & 1.5000 & 0.1500 & 300.0000 & 0.0 & N & 0.0 & N & 0.0 & $\mathbf{N}$ & 0.0 & $\mathbf{N}$ & 300.0000 \\
\hline 24 & $\mathrm{ACL} 474$ & 0.7000 & 0.1000 & 0.7000 & 0.0500 & 300.0000 & 0.0 & N & 0.0 & $\mathbf{N}$ & 0.0 & $\mathbf{N}$ & 0.0 & $\mathbf{M}$ & 250.0000 \\
\hline 24 & ACL 475 & 20.0000 & 0.3000 & 15.0000 & 0.1500 & 5000.00006 & 0.0 & N & 0.0 & $\mathbf{N}$ & 0.0 & N & 10.0000 & & $0.0 \quad L$ \\
\hline 24 & $A C L 476$ & 1.5000 & 0.5000 & 1.5000 & 0.1000 & 700.0000 & 0.0 & H & 0.0 & $\mathbf{N}$ & 0.0 & $\mathbf{N}$ & 0.0 & $\mathbf{N}$ & 300.0000 \\
\hline 24 & $A C L 477$ & 7.0000 & 1.5000 & 0.7000 & 0.3000 & 300.0000 & 0.500 & & 0.0 & $\mathbf{N}$ & 0.0 & $\mathbf{N}$ & 0.0 & $\mathbf{N}$ & 2000.0000 \\
\hline 25 & $\mathrm{ACL} 4 \mathrm{~B} 4$ & 10.0000 & 0.1500 & 0.1000 & 0.0300 & 200.0000 & 2.000 & & 0.0 & $\mathbf{N}$ & 0.0 & $\mathbf{N}$ & 0.0 & $L$ & 150.0000 \\
\hline 26 & $A C L 568$ & 3.0000 & 0.5000 & 0.5000 & 0.0300 & 70.0000 & 0.0 & $\mathbf{N}$ & 0.0 & $\mathbf{N}$ & 0.0 & $\mathbf{N}$ & 0.0 & $\mathbf{N}$ & 100.0000 \\
\hline 24 & $A C L 569$ & 3.0000 & 2.0000 & 10.0000 & 0.3000 & 500.0000 & 0.0 & $L$ & 0.0 & $N$ & 0.0 & $\mathbf{N}$ & 0.0 & $\mathbf{N}$ & 2000.0000 \\
\hline 26 & $\operatorname{ACL} 570$ & 20.0000 & 0.3000 & 7.0000 & 0.1000 & 1000.0000 & 0.0 & $\mathbf{N}$ & 0.0 & $\mathbf{N}$ & 0.0 & N & 0.0 & $L$ & 5000.0000 \\
\hline 36 & $A C L 5 / 1$ & 10.0000 & 3.0000 & 7.0000 & 0.7000 & 1000.0000 & 0.0 & $\mathbf{N}$ & 0.0 & $\mathbf{N}$ & 0.0 & $\mathbf{N}$ & 10.0000 & & $5000.0000 G$ \\
\hline 26 & ACL572 & 3.0000 & 2.0000 & 3.0000 & 0.3000 & 500.0000 & 0.0 & $\mathbf{N}$ & 0.0 & $\mathbf{N}$ & 0.0 & $\mathbf{N}$ & 0.0 & N & 300.0000 \\
\hline 27 & ACL617 & 2.0000 & 1.5000 & 1.5000 & 0.1500 & 200.0000 & 0.0 & $\mathbf{N}$ & 0.0 & $\mathbf{N}$ & 0.0 & $\mathbf{N}$ & 0.0 & $N$ & 1000.0000 \\
\hline 27 & $A C L B 18$ & 1.5000 & 1.0000 & 2.0000 & 0.1500 & 100.0000 & 0.0 & $\mathbf{N}$ & 0.0 & $\mathbf{N}$ & 0.0 & $\mathbf{N}$ & 0.0 & $\mathbf{N}$ & 500.0000 \\
\hline 27 & ACL 619 & 20.00000 & 0.7000 & 1.0000 & 0.0700 & 300.0000 & 1.500 & & 0.0 & $\mathbf{M}$ & 0.0 & $\mathbf{N}$ & 15.0000 & & 150.0000 \\
\hline 27 & ACL 573 & 2.0000 & 1.5000 & 0.3000 & 0.3000 & 300.0000 & 0.0 & $\mathbf{N}$ & 0.0 & $\mathbf{M}$ & 0.0 & $\boldsymbol{H}$ & 0.0 & $\mathbf{N}$ & 300.0000 \\
\hline
\end{tabular}


CRAIG B-5 ROCK SAMPLES

\begin{tabular}{|c|c|c|c|c|c|c|c|c|c|c|c|c|c|c|c|c|c|c|}
\hline & SAMPLE & $B \bar{E} P P$ & $\mathrm{PM}$ & B I & PPY & $\mathrm{CD}$ & PPM & CO PPM & CR PP & & CU PPM & LA & PPM & MO PI & PPM & NB PP & PM & NI PPM \\
\hline 1 & $A C L 320$ & 0.0 & $N$ & 0.0 & $N$ & 0.0 & $N$ & 5.0000 & 15.0000 & & 10.0000 & 0.0 & $N$ & 0.0 & $\mathbf{N}$ & 0.0 & L & 10.0000 \\
\hline $2^{2}+1+3$ & $A C L 325$ & 0.0 & $\mathrm{~N}$ & 0.0 & $\mathrm{~N}$ & 0.0 & $\mathrm{~N}$ & 7.0000 & 15.0000 & & 20.0000 & 0.0 & N & 0.0 & $\mathrm{~N}$ & 10.0000 & & 30.0000 \\
\hline 3 & $A C L 326$ & 0.0 & N & 0.0 & $N$ & 0.0 & $\mathrm{~N}$ & 7.0000 & 30.0000 & & $0.0 \quad \mathrm{~L}$ & 0.0 & N & 0.0 & $L$ & 0.0 & $\mathbf{L}$ & 50.0000 \\
\hline 3 & $A C L 321$ & 0.0 & $\mathbf{L}$ & 0.0 & $\mathrm{~N}$ & 0.0 & $\mathrm{~N}$ & 20.0000 & 150.0000 & & 15.0000 & 0.0 & $\mathbf{N}$ & 0.0 & $\mathrm{~L}$ & 10.0000 & 10 & 100.0000 \\
\hline 3 & $A[L 328$ & 1.0000 & & 0.0 & $\mathbf{N}$ & 0.0 & $\mathrm{~N}$ & 70.0000 & 70.0000 & & 7000.0000 & 0.0 & $\mathbf{N}$ & 15.000 & 00 & 10.0000 & & 70.0000 \\
\hline 4 & $A C L 329$ & 0.0 & $\mathbf{N}$ & 0.0 & N & 0.0 & $\mathbf{N}$ & 20.0000 & 15.0000 & & 30.0000 & 0.0 & $\mathbf{N}$ & 0.0 & $\mathbf{N}$ & 10.0000 & & 20.0000 \\
\hline 5 & $A C L 330$ & 1.0000 & & 0.0 & N & 0.0 & $\mathrm{~N}$ & 7.0000 & 15.0000 & & 15.0000 & 0.0 & $N$ & 0.0 & $\mathbf{L}$ & 0.0 & $\mathbf{L}$ & 20.0000 \\
\hline 5 & $A C\{331$ & 0.0 & $\mathrm{l}$ & 0.0 & $\mathbf{N}$ & 0.0 & $\mathbf{N}$ & 15.0000 & 15.0000 & & 30.0000 & 0.0 & N & 0.0 & L & 10.0000 & & 30.0000 \\
\hline 5 & ACL 332 & 0.0 & $\mathbf{L}$ & 0.0 & $\mathbf{N}$ & 0.0 & $\mathbf{N}$ & 7.0000 & 10.0000 & & 20.0000 & 0.0 & N & 0.0 & L & 0.0 & $\mathbf{L}$ & 30.0000 \\
\hline 5 & $\operatorname{ACL} 333$ & 0.0 & $N$ & 0.0 & v & 0.0 & $\mathbf{N}$ & 5.0000 & 10.0000 & & $0.0 \quad \mathrm{~L}$ & 0.0 & N & 0.0 & N & 0.0 & $\mathbf{L}$ & 15.0000 \\
\hline 5 & ACL 334 & 1.0000 & & 0.0 & $\mathrm{~N}$ & 0.0 & $N$ & 10.0000 & 15.0000 & & 50.0000 & 0.0 & $\mathbf{N}$ & 0.0 & $\mathbf{L}$ & 0.0 & $L$ & 20.0000 \\
\hline 6 & $A C L 335$ & 0.0 & L & 0.0 & $\mathbf{N}$ & 0.0 & $\mathbf{N}$ & $0.0 \quad \mathrm{~L}$ & 20.0000 & & 30.0000 & 0.0 & $\mathbf{N}$ & 0.0 & L & 0.0 & $\mathbf{L}$ & 20.0000 \\
\hline 7 & $A C E 651$ & 0.0 & L & 0.0 & $\mathbf{N}$ & 0.0 & $\mathbf{N}$ & 7.0000 & 20.0000 & & $0.0 \quad \mathrm{~L}$ & 0.0 & N & 0.0 & L & 15.0000 & & 30.0000 \\
\hline 2 & ACE 52 & $u .0$ & $\mathrm{~N}$ & 0.0 & $\mathbf{N}$ & 0.0 & $\mathbf{N}$ & 30.0000 & 20.0000 & & 70.0000 & 0.0 & $\mathbf{N}$ & 0.0 & N & 15.0000 & & 30.0000 \\
\hline 8 & ACL 323 & 0.0 & $N$ & 0.0 & $\mathbf{N}$ & 0.0 & $\mathbf{N}$ & 10.0000 & 20.0000 & & $0.0 \quad L$ & 0.0 & N & 0.0 & L & 10.0000 & & 20.0000 \\
\hline 8 & $A C L 324$ & 0.0 & $v$ & 0.0 & $\mathrm{~N}$ & 0.0 & $\mathbf{N}$ & 15.0000 & 10.0000 & & 0.0 & 0.0 & $\mathbf{N}$ & 0.0 & $\mathbf{N}$ & 10.0000 & & 15.0000 \\
\hline 9 & $A C L 347$ & 0.0 & L & 0.0 & $\mathrm{~N}$ & 0.0 & $N$ & 20.0000 & 50.0000 & & $0.0 \quad \mathrm{~L}$ & 0.0 & N & 0.0 & N & 10.0000 & & 30.0000 \\
\hline 10 & ACL 345 & 0.0 & N & 0.0 & $\boldsymbol{N}$ & 0.0 & $\mathrm{~N}$ & 15.0000 & 30.0000 & & 10.0000 & 0.0 & N & 0.0 & L & 10.0000 & & 30.0000 \\
\hline " & ACL 346 & 0.0 & $N$ & 0.0 & $N$ & 0.0 & $\mathbf{N}$ & 50.0000 & 150.0000 & & 100.0000 & 0.0 & $N$ & 0.0 & $\mathbf{t}$ & 10.0000 & & 70.0000 \\
\hline$/ 2$ & ACL 322 & 0.0 & $N$ & 0.0 & $N$ & 0.0 & $\mathbf{N}$ & $0.0 \quad N$ & 0.0 & $\mathbf{L}$ & 30.0000 & 0.0 & N & 0.0 & $N$ & 0.0 & $\mathbf{L}$ & 10.0000 \\
\hline 13 & ACE 949 & 0.0 & N & 0.0 & N & 0.0 & $\mathrm{~N}$ & 50.0000 & 150.0000 & & 100.0000 & 0.0 & $\mathbf{N}$ & 0.0 & L & 10.0000 & & 50.0000 \\
\hline s & ACE950 & 0.0 & $N$ & 0.0 & $N$ & 0.0 & $N$ & 20.0000 & 100.0000 & & 100.0000 & 0.0 & $N$ & 0.0 & $\bar{N}$ & 0.0 & $\mathbf{L}$ & 30.0000 \\
\hline 14 & $A C L 458$ & 1.5000 & & 0.0 & $v$ & 0.0 & $N$ & 15.0000 & 15.0000 & & 50.0000 & 0.0 & $\mathbf{L}$ & 15.000 & 00 & 10.0000 & & 100.0000 \\
\hline is & $A C L 457$ & 0.0 & $\mathrm{~L}$ & 0.0 & $\mathbf{N}$ & 0.0 & $N$ & 15.0000 & 10.0000 & & 30.0000 & 0.0 & $\mathbf{N}$ & 0.0 & $\mathbf{N}$ & 10.0000 & & 20.0000 \\
\hline 16 & ACL 456 & 0.0 & N & 0.0 & $N$ & 0.0 & $\mathrm{~N}$ & $0.0 \quad \mathrm{~N}$ & 0.0 & $\mathbf{L}$ & $0.0 \quad \mathrm{~N}$ & 0.0 & L & 0.0 & N & 0.0 & $\mathbf{L}$ & 0.0 N \\
\hline$h$ & ACL 454 & 1.5000 & & 0.0 & N & 0.0 & $\mathbf{N}$ & 10.0000 & 20.0000 & & 70.0000 & 0.0 & $\mathbf{L}$ & 5.000 & & 10.0000 & & 30.0000 \\
\hline 7 & ACL 455 & 1.5000 & & 0.0 & N & 0.0 & $\mathbf{N}$ & 70.0000 & 200.0000 & & 100.0000 & 30.00 & 100 & 5.000 & & 15.0000 & & 150.0000 \\
\hline & $A C L 453$ & 1.5000 & & 0.0 & $\mathrm{~N}$ & 0.0 & $\mathbf{N}$ & 15.0000 & 15.0000 & & 100.0000 & 0.0 & $\mathbf{N}$ & 0.0 & $\mathbf{L}$ & 10.0000 & 10 & 20.0000 \\
\hline$s f$ & $\mathrm{ACL} 452$ & 0.0 & $\mathbf{N}$ & 0.0 & $\mathbf{N}$ & 0.0 & $\mathbf{N}$ & $0.0 \quad N$ & 0.0 & $\mathbf{L}$ & $0.0 \quad N$ & 0.0 & $\mathbf{N}$ & 0.0 & $\mathbf{N}$ & 0.0 & $\mathbf{L}$ & $0.0 \mathrm{~N}$ \\
\hline 20 & ACL 460 & 0.0 & $\mathbf{L}$ & 0.0 & N & 0.0 & N & 7.0000 & 0.0 & L & 0.0 & 0.0 & N & 0.0 & $\mathbf{N}$ & 10.0000 & & 7.0000 \\
\hline 20 & $A C L 461$ & 0.0 & $\mathrm{~N}$ & 0.0 & $N$ & 0.0 & $\mathbf{N}$ & 5.0000 & 0.0 & $\mathbf{k}$ & $0.0 \quad \mathrm{~L}$ & 0.0 & $\mathbf{N}$ & 0.0 & $\mathbf{N}$ & 0.0 & $\mathbf{L}$ & 7.0000 \\
\hline $2 t$ & $A C L 46.2$ & 0.0 & $\mathrm{~L}$ & 0.0 & N & 0.0 & N & $0.0 \quad L$ & 0.0 & L & 10.0000 & 0.0 & N & 0.0 & N & 0.0 & \llcorner & 7.0000 \\
\hline 22 & $A C L 463$ & 1.0000 & & 0.0 & $\mathbf{N}$ & 0.0 & $\mathbf{N}$ & 5.0000 & 0.0 & L & 5.0000 & 0.0 & $\mathbf{N}$ & 0.0 & $\mathbf{N}$ & 0.0 & $\mathbf{L}$ & 10.0000 \\
\hline 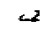 & ACL464 & 0.0 & $\mathrm{k}$ & 0.0 & N & 0.0 & $N$ & 30.0000 & 15.0000 & & 150.0000 & 0.0 & N & 7.000 & & 10.0000 & 10 & 30.0000 \\
\hline 2.2 & ACL 465 & 0.0 & $\mathbf{L}$ & 0.0 & $N$ & 0.0 & $\mathbf{N}$ & $0.0 \quad N$ & 0.0 & L & 20.0000 & 0.0 & N & 7.0000 & & 10.0000 & 10 & 5.0000 \\
\hline 23 & ACL 466 & E. 0000 & & 0.0 & $\mathrm{~N}$ & 0.0 & N & 0.0 & 0.0 & $L$ & 100.0000 & 0.0 & $\mathbf{N}$ & 0.0 & $\mathbf{L}$ & 0.0 & $\mathbf{L}$ & 7.0000 \\
\hline 24 & ACL 474 & 1.0000 & & 0.0 & N & 0.0 & $N$ & $0.0 \quad N$ & 0.0 & $\mathbf{L}$ & 70.0000 & 0.0 & $\mathbf{N}$ & 0.0 & L & 0.0 & $\mathbf{L}$ & 7.0000 \\
\hline 24 & ACL 475 & 1.5000 & & 0.0 & N & 50.00 & 100 & 10.0000 & 10.0000 & & 300.0000 & 0.0 & $\mathbf{N}$ & 15.0000 & & 10.0000 & & 20.0000 \\
\hline 24 & $A C L 476$ & 0.0 & $\mathbf{L}$ & 0.0 & $\mathbf{N}$ & 0.0 & N & $0.0 \quad \mathrm{~N}$ & 0.0 & L & 30.0000 & 0.0 & N & 15.000 & & 10.0000 & & 10.0000 \\
\hline 24 & $A C L 477$ & 0.0 & 1 & 0.0 & $N$ & 0.0 & $N$ & 7.0000 & 50.0000 & & 150.0000 & 0.0 & $\mathbf{N}$ & 30.000 & & 0.0 & $\mathbf{L}$ & 100.0000 \\
\hline 25 & ACL 484 & 0.0 & $N$ & 0.0 & $N$ & 0.0 & N & 10.0000 & 0.0 & $L$ & $0.0 \quad \mathrm{~L}$ & 0.0 & $\mathrm{~N}$ & 0.0 & $\mathrm{~L}$ & 15.0000 & 0 & 15.0000 \\
\hline 26 & $A C L 568$ & 0.0 & $v$ & 0.0 & $N$ & 0.0 & N & $0.0 \quad \mathrm{~N}$ & 0.0 & L & $200.0000^{\circ}$ & 0.0 & $N$ & 0.0 & $\mathbf{N}$ & 10.0000 & & 5.0000 \\
\hline 25 & ACL569 & 0.0 & L & 0.0 & $\mathrm{v}$ & 0.0 & N & 25.0000 & 10.0000 & & 150.0000 & 0.0 & L & 0.0 & $\mathbf{L}$ & 10.0000 & & 30.0000 \\
\hline 2.6 & $A C L 570$ & 0.0 & $N$ & 0.0 & $N$ & 0.0 & $*$ & 5.0000 & 300.0000 & & 10.0000 & 0.0 & $L$ & 0.0 & L & 10.0000 & 0 & 30.0000 \\
\hline 26 & $A C L 571$ & 1.0000 & & 0.0 & $N$ & 0.0 & $\mathrm{~N}$ & 15.0000 & 200.0000 & & 15.0000 & 0.0 & $\mathbf{L}$ & 5.000 & & 0.0 & $\mathbf{L}$ & 50.0000 \\
\hline 26 & $\operatorname{ACL} 572$ & 0.0 & $N$ & 0.0 & N & 0.0 & $\mathrm{~N}$ & 10.0000 & 70.0000 & & 15.0000 & 0.0 & N & 0.0 & $\mathbf{N}$ & 0.0 & $\mathbf{L}$ & 20.0000 \\
\hline 27 & ACL617 & 0.0 & $L$ & 0.0 & $\mathrm{~N}$ & 0.0 & $\mathbf{N}$ & 5.0000 & 30.0000 & & 50.0000 & 0.0 & $\mathbf{L}$ & 10.000 & & 0.0 & $\overline{\mathbf{L}}$ & 70.0000 \\
\hline 27 & ACL 618 & 0.0 & 1 & 0.0 & N & 0.0 & $\mathrm{~N}$ & $0.0 \quad \mathrm{~L}$ & 0.0 & $\mathbf{L}$ & 30.0000 & 0.0 & $\mathbf{N}$ & 5.000 & 0 & 0.0 & $\mathbf{L}$ & 30.0000 \\
\hline 27 & ACL 619 & 0.0 & $\mathrm{~N}$ & 0.0 & $\mathbf{N}$ & 0.0 & $\mathbf{N}$ & 20.0000 & 0.0 & $\mathbf{L}$ & 1500.0000 & 0.0 & L & 10.000 & & 10.0000 & 0 & 70.0000 \\
\hline 27 & ACL573 & 0.0 & $t$ & 0.0 & $\mathbf{N}$ & 0.0 & $\mathbf{N}$ & 7.0000 & 70.0000 & & 15.0000 & 0.0 & $\mathbf{N}$ & 0.0 & $\mathbf{N}$ & 0.0 & $\mathbf{L}$ & 15.0000 \\
\hline
\end{tabular}


CRAIG B-5 ROCK SAMPLES

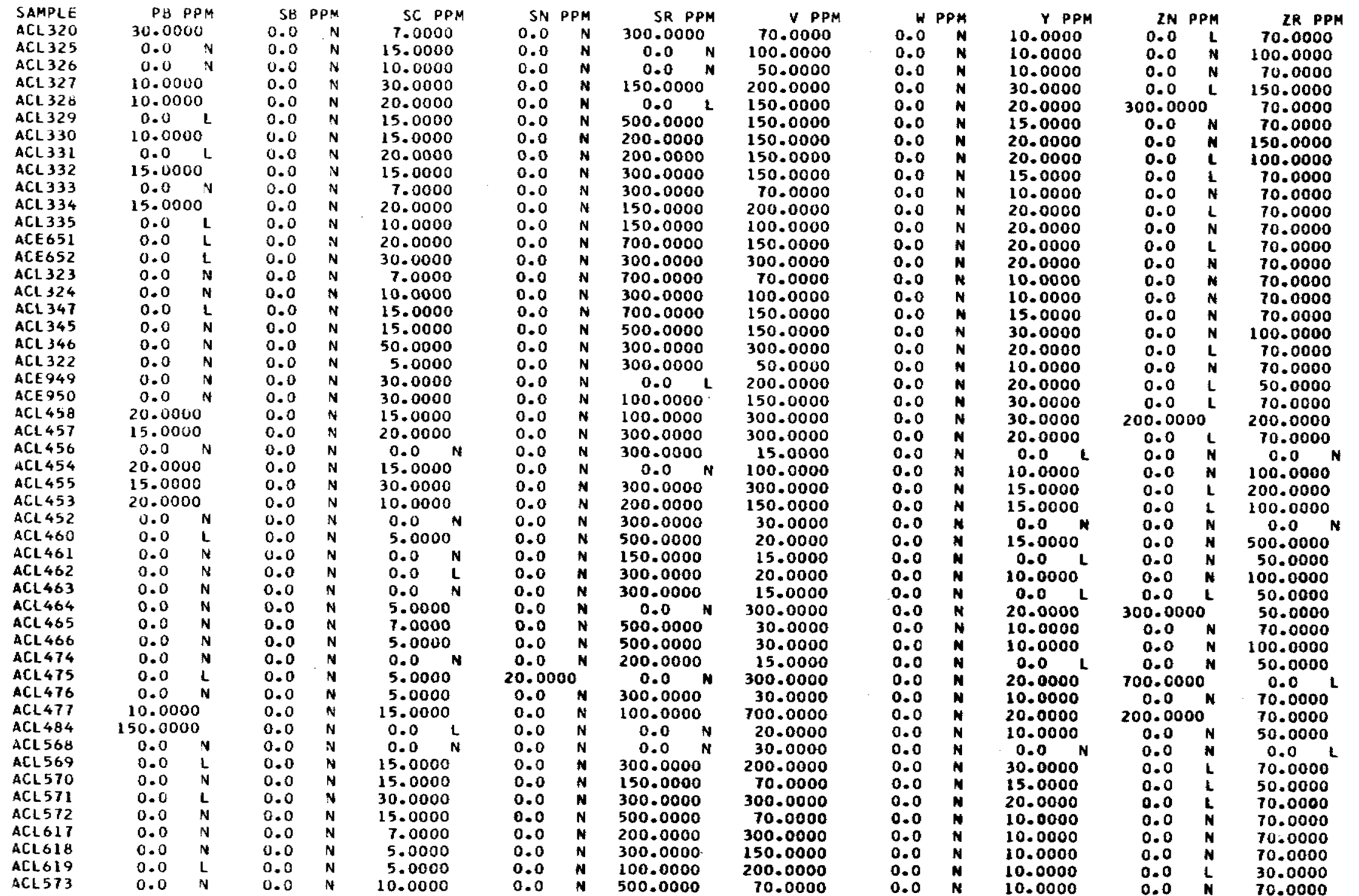


CKAIG B -5 ROCK SAMPLES

\begin{tabular}{|c|c|}
\hline SAMPLE & A1) PPM \\
\hline$A C L 320$ & $0.0200 \mathrm{~L}$ \\
\hline$A C L 325$ & $0.0200 \mathrm{~L}$ \\
\hline$A C L 326$ & $0.0200 \mathrm{~L}$ \\
\hline$A C L 327$ & $0.0200 \mathrm{~L}$ \\
\hline $\mathrm{ACL} 328$ & 0.0600 \\
\hline $\operatorname{ACL} 329$ & $0.0200 \mathrm{~L}$ \\
\hline$A C L 3] C$ & $0.0200 \mathrm{~L}$ \\
\hline$A C L 331$ & $0.0200 \mathrm{~L}$ \\
\hline$A C L 332$ & $0.0200 \mathrm{t}$ \\
\hline$A C L 333$ & $0.0200 \mathrm{~L}$ \\
\hline ACL 334 & $0.0200 \mathrm{~L}$ \\
\hline$A C L 335$ & $0.0200 \mathrm{~L}$ \\
\hline ACE 651 & $0.0200 \mathrm{~L}$ \\
\hline ACE 652 & $0.0200 \mathrm{~L}$ \\
\hline$A C L 323$ & $0.0200 \mathrm{~L}$ \\
\hline ACL 324 & $0.0200 \mathrm{t}$ \\
\hline ACL 347 & $0.0200 \mathrm{~L}$ \\
\hline$A C L 345$ & $0.0200 \mathrm{~L}$ \\
\hline ACL 346 & $0.0200 \mathrm{~L}$ \\
\hline ACL 322 & $0.0200 \mathrm{~L}$ \\
\hline ACE 949 & $0.0200 \mathrm{~L}$ \\
\hline ACE 950 & $0.0200 \mathrm{~L}$ \\
\hline$A C L 458$ & $0.0200 \mathrm{~L}$ \\
\hline$A C L 457$ & $0.0200 \mathrm{~L}$ \\
\hline ACL 456 & $0.0200 \mathrm{~L}$ \\
\hline ACL 454 & $0.0200 \mathrm{~L}$ \\
\hline$A C L 455$ & $0.0200 \mathrm{~L}$ \\
\hline$A C L 45]$ & $0.0200 \mathrm{~L}$ \\
\hline $\operatorname{ACL} 452$ & $0.0200 \mathrm{~L}$ \\
\hline$A C L 460$ & $0.0200 \mathrm{~L}$ \\
\hline$A C L 461$ & 0.02 UOL. \\
\hline$A C L 462$ & $0.0200 \mathrm{~L}$ \\
\hline$A C L 463$ & $0.0200 \mathrm{~L}$ \\
\hline ACL 464 & $0.0200 \mathrm{~L}$ \\
\hline $\mathrm{ACL} 465$ & 0.02001 \\
\hline ACL466 & $0.0200 \mathrm{~L}$ \\
\hline $\mathrm{ACL} 474$ & $0.0200 t$ \\
\hline$A C L 475$ & 0.02004 \\
\hline$A C L 476$ & $0.0200 \mathrm{~L}$ \\
\hline$A C L 477$ & $0.0200 \mathrm{~L}$ \\
\hline $\mathrm{ACL} 484$ & 1.5000 \\
\hline ACL 568 & $0.0200 \mathrm{~L}$ \\
\hline$A C L 569$ & $0.0200 \mathrm{~L}$ \\
\hline$A C L 570$ & 0.02002 \\
\hline$A C L 571$ & 0.02001 \\
\hline ACL 572 & $0.0200 \mathrm{~L}$ \\
\hline ACL 617 & $0.0200 \mathrm{~L}$ \\
\hline ACL 618 & $0.0200 \mathrm{~L}$ \\
\hline ACt619 & $0.0200 \mathrm{~L}$ \\
\hline ACL573 & $0.0200 \mathrm{~L}$ \\
\hline
\end{tabular}


CRAIG B-5 ROCK SAMPLES

\begin{tabular}{|c|c|c|c|c|c|c|c|}
\hline Nimen & SAMPLE & FE PCT & MG PCT & CA PCT & TI PCI & MN PPM & AG \\
\hline $2 x$ & $\mathrm{ACL} 574$ & 2.0000 & 1.5000 & 0.7000 & 0.2000 & 200.0000 & 0.0 \\
\hline 29 & $A C L 620$ & 3.0000 & 3.0000 & 1.5000 & 0.3000 & 300.0000 & 0.5000 \\
\hline 30 & ACL 575 & 5.0000 & 3.0000 & 5.0000 & 0.7000 & 500.0000 & $0.0 \quad \mathrm{~N}$ \\
\hline 30 & $A C L 576$ & 1.5000 & 0.2000 & 1.0000 & 0.0300 & 70.0000 & 0.0 \\
\hline 31 & ACL621 & $\begin{array}{l}3.0000 \\
1.0000\end{array}$ & $\begin{array}{l}3.0000 \\
0.7000\end{array}$ & $\begin{array}{l}1.5000 \\
0.3000\end{array}$ & 0.5000 & 500.0000 & 0.0 \\
\hline 32 & $A C L 485$ & 7.0000 & 3.0000 & 5.0000 & $\begin{array}{l}0.1500 \\
0.3000\end{array}$ & $\begin{array}{l}130.0000 \\
700.0000\end{array}$ & 0.0 \\
\hline 32 & $A C L 480$ & 15.0000 & 1.5000 & 1.5000 & 0.3000 & 200.0000 & 0.5000 \\
\hline
\end{tabular}

$\begin{array}{llll}0.0 & P P M & & \\ 0.0 & N & 0.0 & N \\ 0.0 & N & 0.0 & N \\ 0.0 & N & 0.0 & N \\ 0.0 & N & 0.0 & N \\ 0.0 & N & 0.0 & N \\ 0.0 & N & 0.0 & N \\ 0.0 & N & 0.0 & N \\ 0.0 & N & 0.0 & N\end{array}$

B PPM BA PPM $0.0 N \quad$ N 1500.0000 $20.0000 \quad 3000.0000$ $\begin{array}{lll}0.0 & N & 1500.0000 \\ 0.0 & N & 0.0\end{array}$

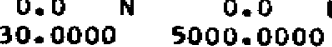
$\begin{array}{ll}30.0000 & 5000.0000 \\ 50.0000 & 3000.0000\end{array}$ 0.0 L 500.0000 $\begin{array}{ll}10.0000 & 200.0000\end{array}$ 
CRAIG B-5 ROCK SAMPLES

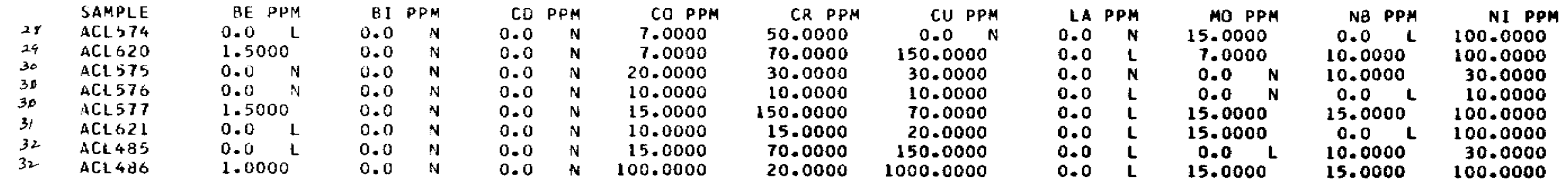


CRAIG 8-5 ROCK SAMPLES

\begin{tabular}{|c|c|c|c|}
\hline & SAMPLE & $P A P P$ & $P M$ \\
\hline 28 & ACL 574 & 0.0 & $N$ \\
\hline 29 & $A C L 620$ & 10.0000 & 0 \\
\hline 50 & ACL 575 & 0.0 & $N$ \\
\hline 30 & ACL 516 & 0.0 & N \\
\hline 30 & $A C L \supset 77$ & 0.0 & $\mathbf{L}$ \\
\hline 31 & ACL 621 & 0.0 & $\mathbf{L}$ \\
\hline 32 & $A C L 485$ & 0.0 & $y$ \\
\hline 32 & & 0.0 & \\
\hline
\end{tabular}

$\begin{array}{lr}\text { SB } & P P M \\ 0.0 & N \\ 0.0 & N \\ 0.0 & N \\ 0.0 & N \\ 0.0 & N \\ 0.0 & N \\ 0.0 & N \\ 0.0 & N\end{array}$

SC PPM

10.0000

15.0000

20.0000

0.0

15.0000

20.0000

15.0000

SN PPM
30.0000

SR PPM

$0.0 \mathrm{~N}$

$\checkmark \mathrm{PPM}$

0.0

100.0000

500.0000

500.0000
150.0000

0.0 N $\quad 0.0$ N 15.0000

0.0 N $200.0000 \quad 700.0000$

0.0 N 0.0 L 500.0000

0.0 i $300.0000 \quad 300.0000$

$\begin{array}{lr} & \\ 0.0 & P P \\ 0.0 & N \\ 0.0 & N \\ 0.0 & N \\ 0.0 & N \\ 0.0 & N \\ 0.0 & N \\ 0.0 & N \\ 0.0 & N\end{array}$

$Y \mathrm{PPH}$

10.0000

30.0000

15.0000

$0.0 \mathrm{~L}$

15.0000

30.0000

20.0000

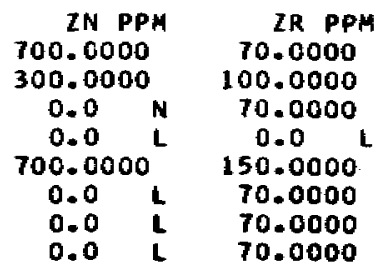


CRAIG B-5 ROCK SAMPLES

SAMPLE

ACL 57

ACL 620

ACL 575

ACL570

ACL 577

Cl621

ACL 485

ACL 486

AU PPM
$0.0200 L$

$0.0200 \mathrm{~L}$

$0.0200 L$

$.0200 \mathrm{~L}$

$0.0200 \mathrm{~L}$

$0.0200 \mathrm{~L}$

$0.0200 \mathrm{~L}$

$0.0200 \mathrm{~L}$

$0.0200 \mathrm{~L}$

E 
FREDUENCY TABLE FOR COLUMN

1 FE PCT)

\begin{tabular}{|c|c|c|}
\hline \multicolumn{3}{|c|}{ LIMIIS } \\
\hline LOWER & & \\
\hline $3.8 \mathrm{E}-02$ & & $5.6 \mathrm{E}-02$ \\
\hline $5.6 E-02$ & & $8.3 E-02$ \\
\hline 8. $3 E-02$ & - & $1-2 E-01$ \\
\hline $1.2 E-01$ & - & $1.8 \mathrm{E}-01$ \\
\hline $1.8 E-01$ & - & $2.5 E-01$ \\
\hline $2.6 E-01$ & - & $3.8 \varepsilon-01$ \\
\hline $3.8 E-01$ & - & $5.6 \mathrm{E}-01$ \\
\hline $5.6 E-01$ & - & B. $3 E-01$ \\
\hline B. $3 E-01$ & - & $1.2 \mathrm{E} \quad 00$ \\
\hline $1.2 E \quad 00$ & - & $1.8 E \quad 00$ \\
\hline $1.8 E 00$ & - & $2.6 \mathrm{E} \quad 00$ \\
\hline $2.6 E 00$ & & $3.8 \mathrm{E} \quad 00$ \\
\hline $3.8 \mathrm{E} \quad 00$ & - & $5.6 \mathrm{E} 00$ \\
\hline $5.6 \mathrm{E} 00$ & - & E. $3 E \quad 00$ \\
\hline $8.3 t$ & - & $1.2 \mathrm{E} \quad 01$ \\
\hline $1.2 \mathrm{E} \quad 01$ & - & $1.8 \mathrm{E} \quad 01$ \\
\hline $1.8 E \quad 01$ & - & $2.6 E \quad 01$ \\
\hline
\end{tabular}

FREP F
0
0
0
0
0
0
1
2
1
7
4
16
4
6
5
6
3

$\begin{array}{ccc}\text { FREQ } & \text { PERCENT } & \text { PERCENT } \\ \text { CUM } & \text { FHEQ } & \text { FREO CUM } \\ 0 & 0.0 & 0.0 \\ 0 & 0.0 & 0.0 \\ 0 & 0.0 & 0.0 \\ 0 & 0.0 & 0.0 \\ 0 & 0.0 & 0.0 \\ 0 & 0.0 & 0.0 \\ 1 & 1.72 & 1.72 \\ 3 & 3.45 & 5.17 \\ 4 & 1.72 & 6.90 \\ 11 & 12.07 & 18.97 \\ 15 & 6.90 & 25.86 \\ 31 & 27.59 & 53.45 \\ 35 & 6.90 & 60.34 \\ 41 & 10.34 & 70.69 \\ 46 & 8.62 & 79.31 \\ 52 & 10.34 & 89.66 \\ 55 & 5.17 & 94.83\end{array}$

HISTUGRAM FOR COLUMN I I FE PCT I

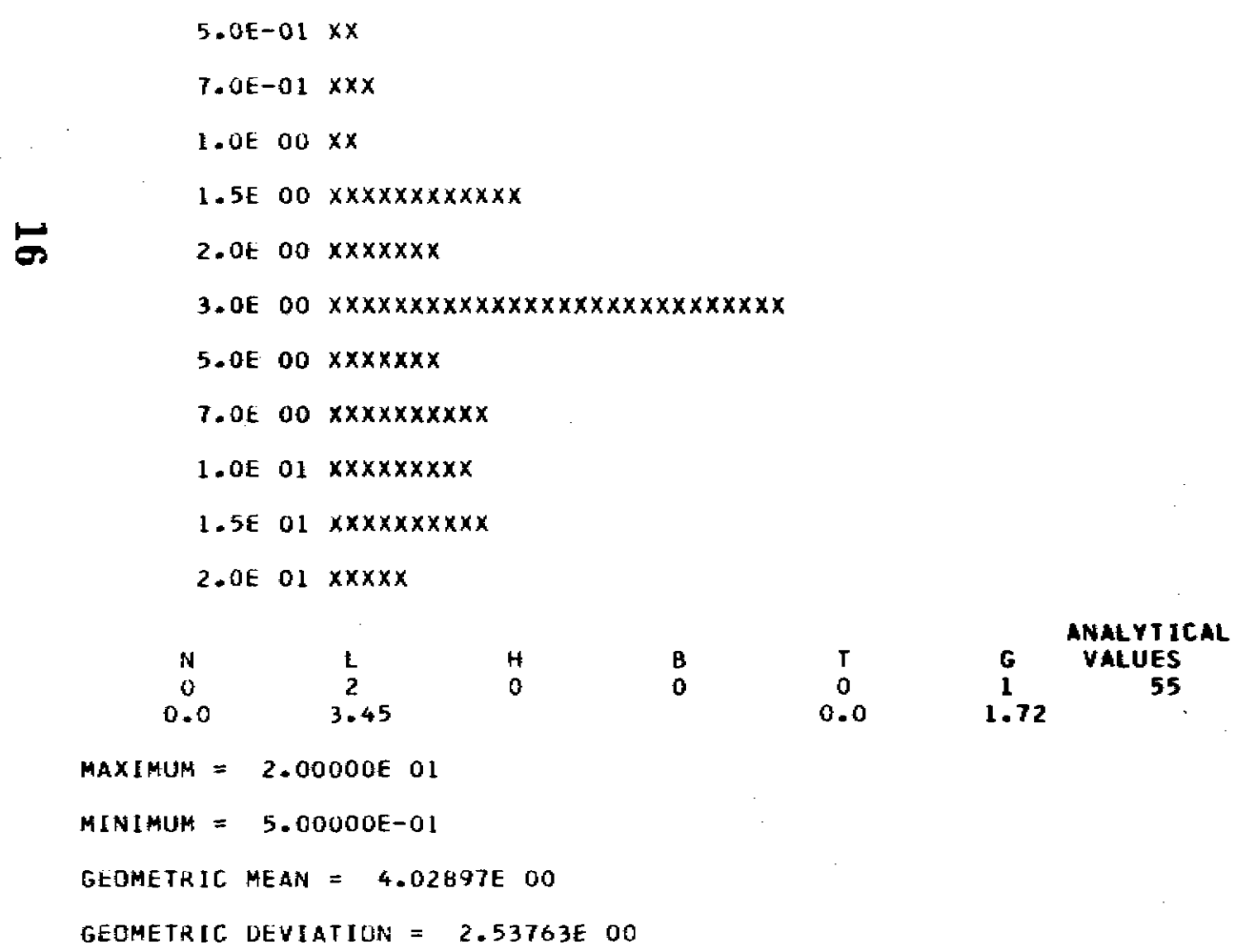


FREQUENCY TABLE FOR COLUMN 2 ( MG PCT)

\begin{tabular}{|c|c|c|c|c|c|c|}
\hline \multicolumn{3}{|c|}{ LIMITS } & FREQ & FREQ & PERCENT & PERCENT \\
\hline $\begin{array}{r}\operatorname{LOWCR} \\
\text { 1. } 8 \mathrm{E}-02\end{array}$ & - & $\begin{array}{l}\text { ERR } \\
2.6 \mathrm{E}-02\end{array}$ & 0 & $\underset{0}{\text { CUM }}$ & $\begin{array}{c}\text { FREQ } \\
0.0\end{array}$ & $\begin{array}{c}\text { FREQ CUM } \\
0.0\end{array}$ \\
\hline $2.6 E-02$ & - & $3.8 \mathrm{E}-02$ & 0 & 0 & 0.0 & 0.0 \\
\hline $3.8 E-02$ & - & $5.6 E-02$ & 0 & 0 & 0.0 & 0.0 \\
\hline $5.6 \mathrm{E}-02$ & - & 8. $3 E-02$ & $\mathbf{I}$ & 1 & 1.72 & 1.72 \\
\hline $8.3 E-02$ & - & $1.2 E-01$ & 1 & 2 & 1.72 & 3.45 \\
\hline $1.2 \mathrm{E}-01$ & - & $1.8 \mathrm{E}-01$ & 2 & 4 & 3.45 & 6.90 \\
\hline $1.8 E-01$ & - & $2.6 E-01$ & 1 & 5 & 1.72 & B.62 \\
\hline $2.6 E-01$ & - & 3. $8 \mathrm{E}-01$ & 3 & 8 & 5.17 & 13.79 \\
\hline $3.8 E-01$ & - & $5.6 E-01$ & 3 & 11 & 5.17 & 18.97 \\
\hline $5.6 E-01$ & - & A. $3 E-01$ & 11 & 22 & 18.97 & 37.93 \\
\hline $8.3 E-01$ & - & $1.2 \mathrm{E} \quad 00$ & 5 & 27 & 8.62 & 46.55 \\
\hline $1.2 \mathrm{E} \quad 00$ & - & 1. BE 00 & 12 & 39 & 20.69 & 67.24 \\
\hline 1.BE 00 & 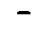 & $2.6 E \quad 00$ & 6 & 45 & 10.34 & 77.59 \\
\hline $2.6 \mathrm{E} \quad 00$ & - & $3.8 \mathrm{E} \quad 00$ & 11 & 56 & 18.97 & 96.55 \\
\hline 3.8E 00 & - & $5.6 \mathrm{E}$ & 1 & 57 & 1.72 & 90.28 \\
\hline $5.6 \mathrm{t} 00$ & - & 8. $3 E \quad 00$ & 1 & 58 & 1.72 & 100.00 \\
\hline
\end{tabular}

HISTUGKAM FOR COLUMN

$21 M G P C T$,

$$
\begin{aligned}
& \text { 7. UE-02 } \times \times \\
& \text { 1. UE-01 } \times \times \\
& 1.5 E-01 \times \times \times \\
& 2.0 E-01 \times \times \\
& \text { 3.0E-01 } \times \times \times \times \times \\
& \text { 5.0E-01 } \times \times \times \times \times
\end{aligned}
$$

7.0E-01 $\times \times \times \times \times \times \times \times \times \times \times \times \times \times \times \times \times \times \times$

1. OE OO $x \times x \times x \times x \times x$

1.5E $00 \quad x \times \times \times \times \times \times \times \times \times \times \times \times \times \times \times \times \times \times \times \times x$

2.0E $00 \times \times \times \times \times \times \times \times \times \times$

3. OE DO $x \times x \times x \times x \times x \times x \times x \times x \times x \times x$

5.0E OOXX

7.0E $00 \times x$

$\begin{array}{ccccccc}N & \text { L } & & & & \text { ANALYTICAL } \\ 0 & 0 & H & B & T & G & \text { VALUES } \\ 0.0 & 0.0 & 0 & 0 & 0 & 0 & 58\end{array}$

MAXIMUM $=7.00000 \mathrm{E}$ UD

MINIMUM $=7.00000 \mathrm{E}-02$

GEUMETRIC MEAN $=1.08411 \mathrm{E} 00$ 
GEDMETRIC DEVIATION $=2.63438 E$ OO 
3 I CA PCT

\begin{tabular}{|c|c|c|c|c|c|}
\hline \multicolumn{2}{|c|}{$\begin{array}{c}\text { LIMIIS } \\
\text { L }\end{array}$} & FREQ & $\begin{array}{l}\text { FREQ } \\
\text { CUM }\end{array}$ & $\begin{array}{c}\text { PERCENT } \\
\text { FREQ }\end{array}$ & $\begin{array}{l}\text { PERCENT } \\
\text { FREO CUM }\end{array}$ \\
\hline 3. $8 E-02$ & $-\quad 5.6 \mathrm{E}-02$ & 0 & 0 & 0.0 & 0.0 \\
\hline $5.6 \mathrm{E}-02$ & $8.3 E-02$ & 0 & 0 & 0.0 & 0.0 \\
\hline $8.3 E-02$ & $1.2 \mathrm{E}-01$ & 1 & 1 & 1.72 & 1.72 \\
\hline $1.2 E-01$ & $1 \cdot B E-01$ & 1 & 2 & 1.72 & 3.45 \\
\hline $1 . \Delta E-01$ & $2.6 E-01$ & 0 & 2 & 0.0 & 3.45 \\
\hline $2.6 E-01$ & 3. $8 E-01$ & 2 & 4 & 3.45 & 6.90 \\
\hline 1. $8 E-01$ & $5.6 \mathrm{E}-01$ & 1 & 5 & 1.72 & 8.62 \\
\hline $5.6 E-01$ & $8.3 E-01$ & 11 & 16 & 18.97 & 27.59 \\
\hline $8.3 E-01$ & $1.2 \mathrm{E} \quad 00$ & 5 & 21 & 8.82 & 36.21 \\
\hline $1.2 E 00$ & $1.8 \mathrm{E} \quad 00$ & 9 & 30 & 15.52 & 51.72 \\
\hline $1.8 E$ & $2.6 \mathrm{E}$ & 5 & 35 & 8.62 & 60.34 \\
\hline $2.6 \mathrm{E}$ & $3.8 E$ & 6 & 41 & 10.34 & 70.69 \\
\hline $3.8 \mathrm{E} 00$ & $5.6 \mathrm{E}$ & 8 & 49 & 13.79 & 64.48 \\
\hline $5.6 \mathrm{E} \quad 00$ & B. $3 \mathrm{E}$ & 4 & 53 & 6.90 & 91.38 \\
\hline B. 3E 00 & $1.2 E$ & 1 & 54 & 1.72 & 93.10 \\
\hline $1.2 E \quad 01$ & $1.8 \mathrm{E} \quad 01$ & 2 & 56 & 3.45 & 96.55 \\
\hline
\end{tabular}

HISTOGRAM FOR COLUMN

3 ( CA PCT )

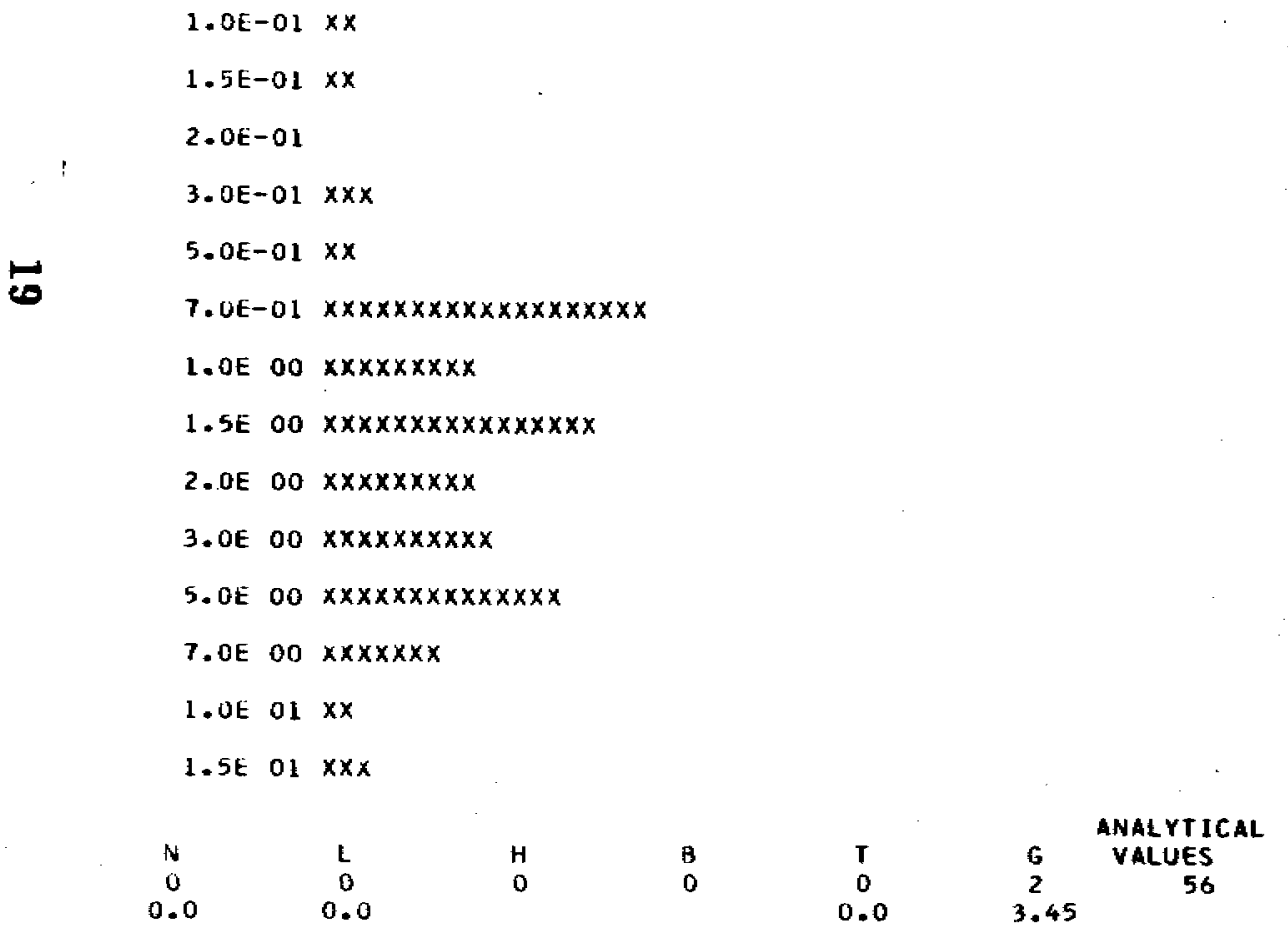

MAXIMUM $=1.50000 E 01$

MINIMUM $=1.00000 \mathrm{E}-0$ 
GEOMETRIC MEAN $=1.73512 \mathrm{E}$ ON

GLOMETRIC UEVIATION $=2.93186$ OO

\section{ง}




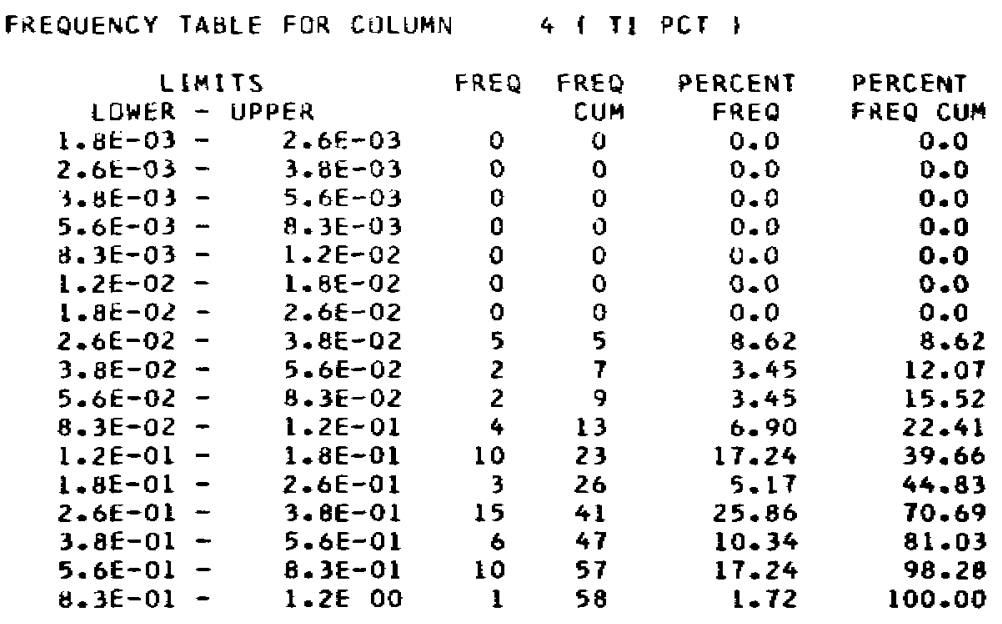

HISTOGRAM FOR COLUMN

4 I TI PCT ।

3. $0 E-02 \times x \times x \times x \times x \times$

5. OE-02 $\times \times X$

7.OE-02 $\times \times x$

1. $0 E-01 \times \times \times \times \times \times x$

N

\subsection{E-01 $\times \times \times \times \times \times \times \times \times \times \times \times \times \times \times \times x$ \\ 2. OE-01 $\times \times \times \times x$ \\ 3. $0 E-01 \quad x \times x \times x \times x \times x \times x \times x \times x \times x \times x \times x \times x \times x \times x x$}

5. OE-01 $\times \times \times \times \times \times \times \times \times \times$

7. OE-01 $\times \times \times \times \times \times \times \times \times \times \times \times \times \times \times \times x$

1. OE OO $\times X$

$\begin{array}{cc}\text { N } & 1 \\ 0 & 0 \\ 0.0 & 0.0 \\ \text { AXIMUM }= & 1.00000 E 00 \\ \text { INIMUM }= & 3.00000 E-02\end{array}$

GEOMETRIC MEAN $=2.20692 \mathrm{E}-01$

GEOMETRIC DEVIATION $=2.59946 E 00$ 
FKEQUENCY TABLE FOR COLUMN 5 ( MN PPM)

\begin{tabular}{|c|c|c|c|c|c|c|c|c|}
\hline \multicolumn{5}{|c|}{$\begin{array}{c}\text { LIMITS } \\
\text { LCWER - UPPER }\end{array}$} & FREO & $\begin{array}{l}\text { FREQ } \\
\text { CUM }\end{array}$ & $\begin{array}{l}\text { PERCENT } \\
\text { FREQ }\end{array}$ & $\begin{array}{l}\text { PERCENT } \\
\text { FREQ CUM }\end{array}$ \\
\hline 8. $3 E$ & 00 & - & $1.2 \mathrm{E}$ & 02 & 0 & 0 & 0.0 & 0.0 \\
\hline $1.2 \mathrm{E}$ & 01 & - & $1.8 \mathrm{E}$ & 01 & 0 & 0 & 0.0 & 0.0 \\
\hline $1.8 E$ & 01 & - & $2.6 E$ & 01 & 0 & 0 & 0.0 & 0.0 \\
\hline $2.6 \mathrm{E}$ & 01 & - & $3.8 E$ & 01 & 0 & 0 & 0.0 & 0.0 \\
\hline 3. $8 \mathrm{E}$ & 01 & - & $5.6 E$ & of & 0 & 0 & 0.0 & 0.0 \\
\hline $5.6 \mathrm{E}$ & 01 & - & $8.3 E$ & 01 & 4 & 4 & 6.90 & 6.90 \\
\hline 8. 3E & 01 & - & $1.2 \mathrm{E}$ & 02 & 3 & 7 & 5.17 & 12.07 \\
\hline $1.2 \mathrm{E}$ & 02 & - & $1.8 E$ & 02 & 2 & 9 & 3.45 & 15.52 \\
\hline $1 . B E$ & 02 & - & $2.6 \mathrm{E}$ & 02 & 4 & 13 & 6.90 & 22.41 \\
\hline $2.6 \mathrm{E}$ & 02 & - & $3.8 \mathrm{EE}$ & 02 & 9 & 22 & 15.52 & 37.93 \\
\hline $3.8 \mathrm{E}$ & 02 & - & $5.6 \mathrm{E}$ & 02 & 11 & 33 & 18.97 & 56.90 \\
\hline $5.6 E$ & 02 & - & $8.3 \mathrm{E}$ & 02 & 6 & 39 & 10.34 & 67.24 \\
\hline$\cdot 3 E$ & 02 & - & $1.2 \mathrm{E}$ & 03 & 7 & 46 & 12.07 & 79.31 \\
\hline $2 E$ & 03 & - & $1.8 \mathrm{E}$ & 03 & 10 & 56 & 17.24 & 96.55 \\
\hline
\end{tabular}

HISTOGRAM FOR COLUMN

5 ( MN PPM )

7. OE $01 \times \times \times \times \times \times \times$

1. OE $02 \times \times \times \times \times$

1.5E $02 \times \times x$

$2.0 \mathrm{O} 02 \times \times \times \times \times \times \times$

3.0E $0.2 \times x \times x \times x \times x \times x \times x \times x \times x$

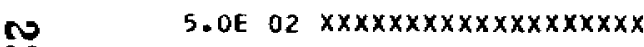

7. OE $02 \times x \times x \times x \times x \times x$

1.0E $03 \quad \times \times \times \times \times \times \times \times \times \times \times x$

1.5E $03 \quad \times \times \times \times \times \times \times \times \times \times \times \times \times \times \times \times \times$

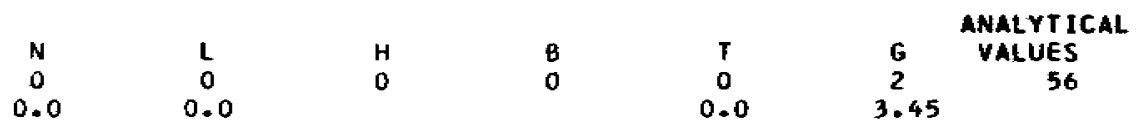

MAXIMUM $=1.50000 E_{03}$

MINIMUM $=7.00000 E 01$

GEDMEIRIC MEAN $=4.53169 E 02$

GEOMETRIC DEVIATION $=2.53053$ EO 
FREOUENCY TABLE FOR COLUMN

6 I AC. PPM J

\begin{tabular}{|c|c|c|c|c|c|}
\hline \multicolumn{2}{|c|}{ LIMITS } & FREQ & $\begin{array}{l}\text { FREQ } \\
\text { CUM }\end{array}$ & $\begin{array}{c}\text { PERCENT } \\
\text { FREQ }\end{array}$ & $\begin{array}{l}\text { PERCENT } \\
\text { FREQ CUM }\end{array}$ \\
\hline $3.8 E-01-$ & $-\quad 5.6 E-0 I$ & 4 & 4 & 6.90 & 6.90 \\
\hline $5.6 E-01-$ & B. $3 E-01$ & i & 3 & 1.72 & 8.62 \\
\hline 8. $3 t-01$. & $1.2 \mathrm{E} \quad 00$ & 0 & 5 & 0.0 & 8.62 \\
\hline $1.2 \mathrm{E} \quad 00-$ & $1.8 \mathrm{E}$ & 1 & 6 & 1.72 & 10.34 \\
\hline 1. $8 \mathrm{E} \quad 00$ & $2.6 \mathrm{E} \quad 00$ & 1 & 7 & 1.72 & 12.07 \\
\hline
\end{tabular}

HISTOGRAM FUR COLUMN

\section{5. $0 \mathrm{E}-01 \times \times \times \times \times \times \times$}

7. OE-OL $\mathrm{XX}$

1. $0 \mathrm{E} \quad 00$

1.5E $00 \times X$

$2.0 E$ OO $X \times$

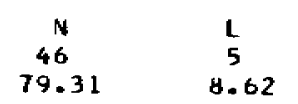

MAXIMUM $=2.00000 E$ OO

MINIMUM $=5.00000 E-01$

S

GEOMEIRIC MEAN $=7.48194$ E-01

GEOMETRIC DEVIATION $=1.80811 E 00$
ANALYTICAL

VALUES. 
frequency table fur COLUMN

7 ( AS PPM,

$$
\begin{gathered}
\text { LIHITS } \\
\text { LUWER - UPPER }
\end{gathered}
$$

$$
\text { FRE }
$$

FREQ PERCEN

LUWER - UPPER

CUM

FREO

PERCENT

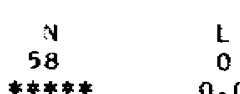

B

AXIMUM $=-9.99900 E 48$

\section{MINIMUM $=9.99900$ E 48}

GEOMETRIC MEAN $=9.99900 E 48$

GEUMETRIC DEVIATION $=9.99900$ E 48 
FREQUENCY TABLE FOR COLUMN

d I AU PPH

\begin{tabular}{|c|c|c|c|c|}
\hline $\begin{array}{c}\text { LIMITS } \\
\text { LOWER - UPPER }\end{array}$ & FREQ & $\begin{array}{r}\text { FREQ } \\
\text { CUM }\end{array}$ & $\begin{array}{c}\text { PERCENT } \\
\text { FREQ }\end{array}$ & $\begin{array}{l}\text { PERCENT } \\
\text { FREQ CUM }\end{array}$ \\
\hline
\end{tabular}

$\begin{array}{cc}1 & 1 \\ 58 & 0 \\ * * * * * & 0.0\end{array}$

$\begin{array}{cc}B & T \\ 0 & 0 \\ & 0.0\end{array}$

ANALYTICAL

XIMUH $=-9.99900 E 48$

MINIMUM $=9.99900 E 48$

GEOMETRIC MEAN $=9.99900 E 48$

GEOMETRIC DEYIATION = 9.99900E $4 \mathrm{~g}$ 


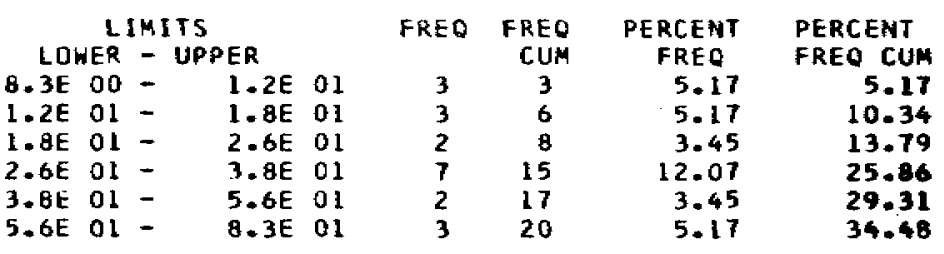

HISTOGRAM FOR COLUMN

1. OE $01 \times \times \times \times x$

1.5E O1 $\times \times \times \times \times$

2. OE $01 \times x \times$

3.0E $01 \quad x \times \times \times \times \times \times \times \times \times \times x$

5. OE O1 $\times \times x$

7.OE $01 \times x \times x \times x$

$\begin{array}{ccccccc}N & L & H & B & T & G & \begin{array}{c}\text { ANALYTICAL } \\ \text { VALUES }\end{array} \\ 28 & 10 & 0 & 0 & 0 & 0 & 20\end{array}$

MAXIMUH $=7.00000 E 01$

0 MINImum $=1.00000$ ol

GEOMETRIC MEAN $=2.63127 E$ Ol

GEOMETRIC DEVIATION = 1. B91BBE 00 
FREQUENCY TABLE FOR COLUMN

10 BA PPM ।

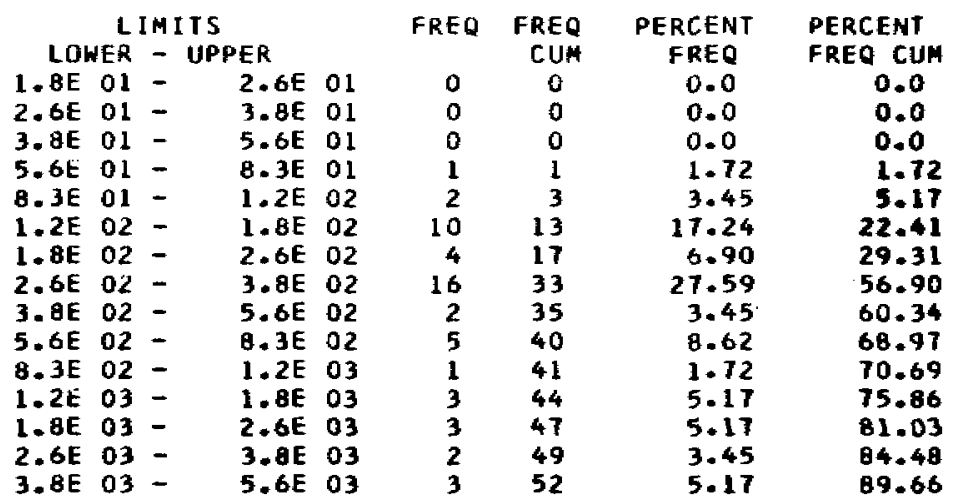

HISTOGRAM FOR COLUMN 10 ( BA PPM )

7. OE O1 XX

1. OE $02 \times x \times$

1. SE $02 \times x \times x \times x \times \times x \times x \times x \times x \times x x$

2. OE $02 \times \times \times \times \times \times x$

3. OE O2 $\times x \times x \times x \times x \times x \times x \times x \times x \times x \times x \times x \times x \times x \times x$

I $5.0 E 02 \times x \times$

T. OE $02 \times \times \times \times \times \times \times \times \times$

1. OE $03 \times x$

$1.5 E 03 \times \times \times \times x$

2. OE $03 \times \times \times \times x$

3. OE $03 \times \times X$

5.0E $03 \times \times \times \times x$

$\begin{array}{ccccccc}N & L & H & & & & \begin{array}{c}\text { ANALYTICAL } \\ \text { VALUES } \\ 0\end{array} \\ 0.0 & 5 & 0 & 0 & 0 & \mathbf{T} & 52\end{array}$

MAXIMUM $=5.00000 E 03$

MINIMUM $=7.00000 E 01$

GEOHETRIC MEAN $=4.22625 \mathrm{E} 02$

GEOMETRIC DEVIATION $=3.0217 T$ EO 
FREQUENCY TABLE FOR COLUMN

$11 \mid$ BE PPM 1

LIMITS

$\begin{array}{lll}\text { LOWER - UPPER } & \text { FREQ FREQ PERCEN } \\ \text { CUM } & \text { FREQ }\end{array}$

A. 3E-01 - $1.2 E 00$

P.3E-O1
$1.2 E 00-1.2 E 00$

8 a

1 ( BE PPM)

HISTUGRAM FOR COLUMN

$x \times x \times x \times$

1. $5 E \quad 00 \quad x \times \times \times \times \times \times \times \times \times \times \times$

$\begin{array}{ccccccc}N & L & H & & & & \text { ANALYTICAL } \\ 23 & 20 & H & B & T & G & \text { VALUES } \\ 39.66 & 34.48 & 0 & 0 & 0 & 0 & 15\end{array}$

MAXIMUM $=2.50000 E 00$

MINImUM $=1.00000 E 00$

GEOMETRIC MEAN $=1.20830 E 00$

GEOMETRIC DEVIATION = 1.23291 EO 
FREQUENCY TABLE FOR COLUMII

12 | BI PPM ।

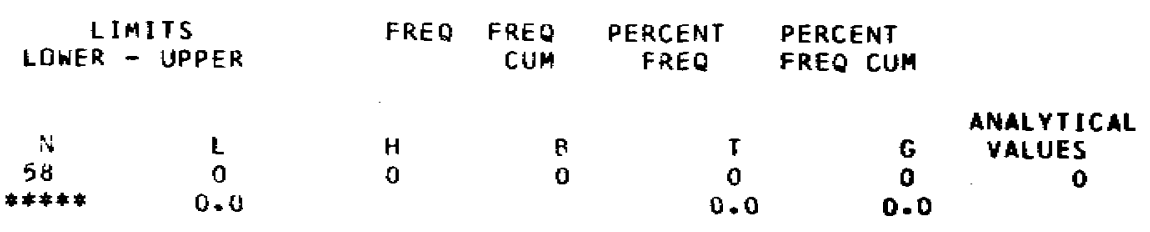

MAXIMUM $=-9.99400 E 40$

MINIMUH $=9.99900 E 48$

GLQMETRIC MEAN = 9.99900 E 48

GEOMETRIC DEVIATION $=9.99900$ E 48 
FREQUENCY TABLE FOR COLUMN

$$
\begin{aligned}
& \text { EIMI IS } \\
& \text { LUWER - UPPER } \\
& \text { 1.QE } 01 \text { - 2.6E OI } \\
& \text { 2.6E OL - 3.BE OI } \\
& \text { 3. } \mathrm{BE} 01-5.6 \mathrm{E} \mathrm{O1} \\
& \text { CUM }
\end{aligned}
$$

HISTOGRAM FOR COLUMH

13 (CO PpH )

$$
\text { 5. OE } 01 \times x
$$

$$
\begin{array}{rcc}
\text { N } & \text { L } & \text { H } \\
98.28 & 0 & 0 \\
\text { MAXIMUH }= & 0.0 & \\
\text { MINIMUM }= & 5.00000 \mathrm{E} 01
\end{array}
$$

GEOHETR IC MEAN $=4.999996$ OI

GEDMETHIC UEVIATIUN $=9.99900 E 48$

$\begin{array}{cc}T & G \\ 0 & 0 \\ 0.0 & 0.0\end{array}$

B

ANALYTICAL

VALUES

1 


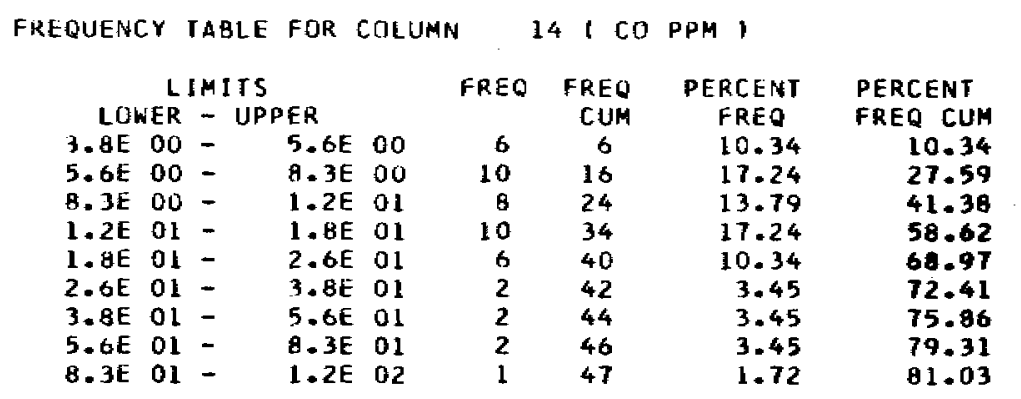

HISTOGRAM FOR COLUMN 14, CO PPM)

5.0E $00 \quad x \times \times \times \times \times \times \times x \times$

7.0E $00 \quad x \times x \times x \times x \times x \times x \times x \times x \times x$

1. OE O1 $\times \times \times \times \times \times \times \times \times \times \times \times \times \times$

1. $5 E$ ol $\times x \times x \times x \times x \times x \times x \times x \times x \times$

$2.0 E \quad 01 \quad \times x \times x \times x \times x \times x$

3. OE OI $X X X$

5. OE O1 $\times \times x$

7. OE $01 \quad \times \times x$

$\omega$

$1.0602 \times x$

$\begin{array}{cc}N & L \\ 13.79 & 3 \\ 5.17\end{array}$

$H$
0

$\mathbf{B}$
$\mathbf{0}$

$\begin{array}{cc}T & 6 \\ 0 & 0 \\ 0.0 & 0.0\end{array}$

ANALYTICAL

VALUES

MAXIMUM $=1.00000 E 02$

MINIMUM $=5.00000 E 00$

GEOMETRIC MEAN $=1.29356 \mathrm{E}$ OI

GEOMETRIC DEVIATION $=2.12201 E$ OO 


\begin{tabular}{|c|c|c|c|c|c|c|c|c|}
\hline \multicolumn{5}{|c|}{ LINITS } & FREQ & $\begin{array}{l}\text { FREQ } \\
\text { CUM }\end{array}$ & $\begin{array}{c}\text { PERCENT } \\
\text { FREQ }\end{array}$ & $\begin{array}{l}\text { PERCENT } \\
\text { FREQ CUM }\end{array}$ \\
\hline $3.8 \mathrm{E}$ & 00 & - & $5.6 t$ & 00 & 0 & 0 & 0.0 & 0.0 \\
\hline $5.6 \mathrm{t}$ & 00 & - & 8. $3 \mathrm{E}$ & 00 & 0 & 0 & 0.0 & 0.0 \\
\hline 6. $3 \mathrm{E}$ & 00 & - & $1.2 \mathrm{E}$ & 01 & 7 & J & 12.07 & 12.07 \\
\hline $1.2 E$ & 01 & - & $1.8 E$ & 01 & 10 & 17 & 17.24 & 29.31 \\
\hline $1.8 E$ & 01 & - & $2.6 E$ & 01 & 6 & 23 & 10.34 & 39.66 \\
\hline $2.6 E$ & 01 & - & $3 . \mathrm{BE}$ & 01 & 4 & 27 & 6.90 & 46.55 \\
\hline $3.8 \mathrm{E}$ & 01 & - & $5.6 \mathrm{E}$ & 01 & 3 & 30 & 5.17 & 51.72 \\
\hline $5.6 \mathrm{E}$ & 01 & - & B. $3 E$ & OL & 5 & 35 & 8.62 & 60.34 \\
\hline $8.3 E$ & 01 & - & $1.2 \mathrm{E}$ & 02 & 1 & 36 & 1.72 & 62.07 \\
\hline $1.2 \mathrm{E}$ & 02 & - & $1.8 \mathrm{E}$ & 02 & 4 & 40 & 6.90 & 68.97 \\
\hline $1.8 E$ & 02 & - & $2.6 E$ & 02 & 2 & 42 & 3.45 & 72.41 \\
\hline $2.6 E$ & 02 & - & $3.8 E$ & 02 & 1 & 43 & 1.72 & 74.14 \\
\hline
\end{tabular}

HISTOGRAM FOR COLUMN 15 ( CR PPM ,

1. OE OL $x \times \times \times \times \times \times \times \times \times \times x$

1. $5 \mathrm{E} \quad 01 \quad \times \times \times \times \times \times \times \times \times \times \times \times \times \times \times \times \times \times x$

2. OE $01 \times \times \times \times \times \times \times \times x \times x$

3.0t ol $\times \times \times \times \times \times x$

5. OE $01 \times \times \times \times x$

7.OE DI $\times x \times x \times x \times \times x$

$\omega$

1. OE $02 \times x$

1.5E $02 \times x \times x \times x \times$

$2.0 E 02 \times \times x$

3. OE $02 \times x$

$\begin{array}{ccccccc}M & L & H & & & & \text { AMALYTICAL } \\ 0 & 15 & 0 & 0 & T & 6 & \text { VALUES } \\ 0.0 & 25.86 & & & 0 & 0 & 43\end{array}$

MAXIMUM $=3.00000 E 02$

MINIMUM $=1.00000 E$ OI

GEOMETRIC MEAN $=3.17607 E$ OI

GEOMETRIC DEVIATION $=2.74369 E$ OO 
FREQUENCY IABLE FOR COLUMN $161 \mathrm{CU}$ PPM I

\begin{tabular}{|c|c|c|c|c|c|c|c|}
\hline \multicolumn{4}{|c|}{ LIMITS } & FREO & $\begin{array}{r}\text { FREQ } \\
\text { CUH }\end{array}$ & $\begin{array}{c}\text { PERCENT } \\
\text { FREO }\end{array}$ & $\begin{array}{l}\text { PERCENT } \\
\text { FREQ CUM }\end{array}$ \\
\hline $3.8 E$ & 00 & $-\quad 5.6 E$ & 00 & 1 & 1 & 1.72 & 1.72 \\
\hline $5.6 \mathrm{E}$ & 00 & B. $3 E$ & No & 0 & i & 0.0 & 1.72 \\
\hline A. $3 E$ & 00 & $1.2 \mathrm{E}$ & 01 & 5 & 6 & 8.62 & 10.34 \\
\hline $1.2 E$ & 01 & $1.8 \mathrm{E}$ & ol & 5 & 11 & 8.62 & .97 \\
\hline 1. at & OI & $2.6 E$ & 01 & 4 & 15 & 6.90 & \\
\hline $2.6 \mathrm{E}$ & 01 & $3.8 \mathrm{E}$ & OI & 8 & 23 & 13.79 & \\
\hline $3.8 \mathrm{E}$ & 01 & $5.6 \mathrm{E}$ & 01 & 3 & 26 & 5.17 & \\
\hline $5.6 E$ & 01 & B. $3 E$ & 01 & 4 & 30 & 6.90 & \\
\hline 8. 3E & 01 & $1.2 E$ & 02 & b & 36 & 10.34 & 62.07 \\
\hline $1.2 E$ & 02 & $1.8 E$ & 02 & 5 & 41 & 8.62 & .69 \\
\hline $1.8 E$ & 02 & $2.6 E$ & 02 & I & 42 & 1.72 & .41 \\
\hline $2.6 E$ & 02 & $3.8 t$ & 02 & 1 & 43 & 1.72 & 74.14 \\
\hline $3.8 \mathrm{E}$ & 02 & $5.6 \mathrm{E}$ & 02 & 0 & 43 & 0.0 & 74.14 \\
\hline $5.6 E$ & 02 & $8.3 E$ & 02 & 0 & 43 & 0.0 & 74.14 \\
\hline $8.3 E$ & 02 & $1.2 \mathrm{E}$ & 03 & 1 & 44 & 1.72 & 75.86 \\
\hline $1.2 \mathrm{E}$ & 03 & $1.8 E$ & 03 & 1 & 45 & 1.72 & 77.59 \\
\hline $1.8 E$ & & $2.6 E$ & 03 & 0 & 45 & 0.0 & 77.59 \\
\hline $2.6 E$ & 03 & $3.8 E$ & 03 & 0 & 45 & 0.0 & 77.59 \\
\hline $3.8 E$ & 03 & $5.6 E$ & 03 & 0 & 45 & 0.0 & 77.59 \\
\hline $5 \mathrm{BF}$ & 03 & B. $3 E$ & 03 & 1 & 46 & 1.72 & \\
\hline
\end{tabular}

HISTOGRAM FOR COLUMN 16 (CU PPH)

$5.0 E$ DO $\times x$

$7.0 \mathrm{E} \quad 00$

1. 0 OE $01 \times \times \times \times \times \times \times \times x$

$\omega$

2. OE $01 \quad x \times \times \times \times x \times$

3.0E $01 \times \times \times \times \times \times \times \times \times \times \times \times \times \times x$

5.OE $01 \times \times \times \times x$

7.0E $01 \times \times \times \times \times \times \times$

1. OE $02 \times \times \times \times \times \times \times \times \times x$

1.5E $02 \times \times \times \times \times \times \times x \times$

$2.0 E \quad 02 \times x$

$3.0 \mathrm{E} \mathrm{O2XX}$

5.0E 02

$7.0 E \quad 02$

1. OE $03 \quad x X$

1.5E $03 x x$

$2.0 \mathrm{E} \quad 03$ 


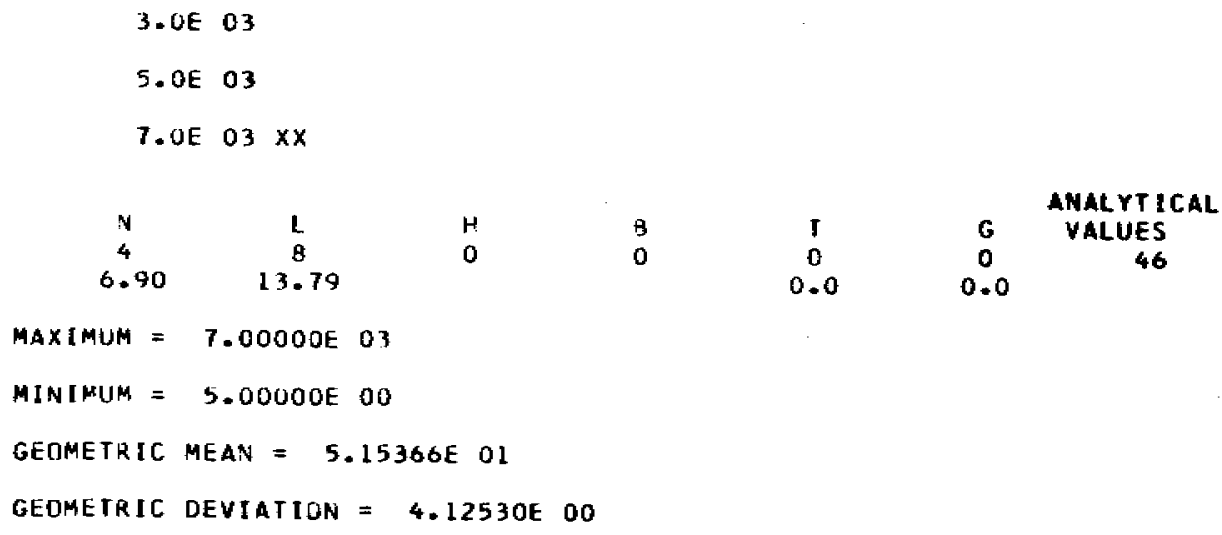$$
46
$$$$
\text { MAXIMUM }=7.00000 E 03
$$$$
\text { MINIMUM = 5.00000E OO }
$$$$
\text { GEOMETRIC MEAN }=5.15366 E \text { OI }
$$$$
\text { GEOMETRIC DEVIATION }=4.12530 \text { OO }
$$

$\omega$ 
FREOUENCY TABLE FUR COLUMN

17 I LA PPM

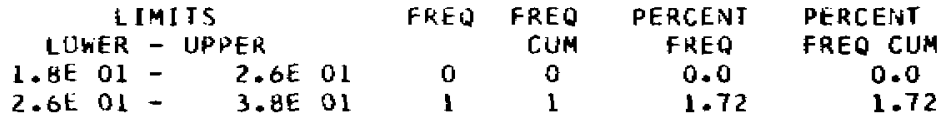

HISTUGRAM FOR COLUMN 17 I LA PPM

3.0 O OI $\times x$

$\begin{array}{ccccccc}N & L & H & & & & \text { ANALYTICAL } \\ 43 & 14 & 0 & 0 & 1 & 0 & \text { VALUES } \\ 74.14 & 24.14 & & & 0.0 & 0.0 & 1\end{array}$

MAXIMUM $=3.00000 E$ OL

MINIMUM $=3.00000 E 01$

GEOMETRIC MEAN $=3.00000$ Ol

GLOMETRIC DEVIATIUN $=9.99900 E 48$ 


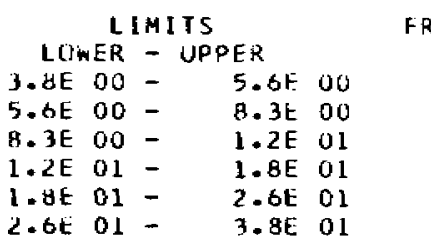

FREO FRE
4
3
2
8
0
1

6.90

$\begin{array}{rrr}4 & 6.90 & 6.90 \\ 7 & 5.17 & 12.07\end{array}$

$9 \quad 3.45 \quad 15.52$

$7 \quad 13.79 \quad 29.31$

$18 \quad 0.0 \quad 29.31$

HISTOGRAM FOR COLUMN IO I MO PPM I

5. OE $00 \times \times \times \times \times \times \times$

7. Ot $00 \quad \times \times \times \times x$

$1.0 E$ OI $\times \times \times$

1.5t $01 \times \times \times \times \times \times \times \times \times \times \times \times \times \times$

2. OE OI

$3.0 \mathrm{E} \quad 01 \times \mathrm{X}$

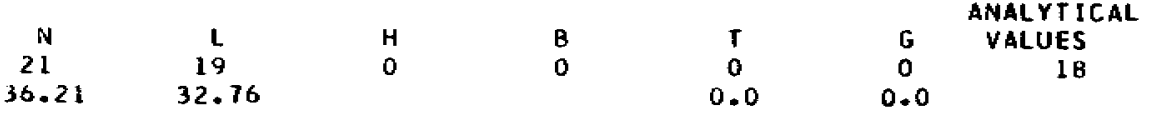

MAXIMUM $=3.00000 E 01$

MINIMUM $=5.00000 E$ DO

GEOMETRIC MEAN $=1.02814 \mathrm{E} 01$

GEOMETRIC OEVIATION $=1.70236 E 00$ 
FREQUENCY IABLE FOR COLUMAN 19 ( NB PPM ,

$\begin{array}{cccccr}\text { LIMITS } & \text { FREQ } & \text { FREQ } & \text { PERCENT } & \text { PERCENT } \\ \text { LOHER - UPPER } & & \text { CUM } & \text { FREQ } & \text { FREQ CUM } \\ \text { A.3E OU - } & 1.2 E \text { OI } & 27 & 27 & 46.55 & 46.55 \\ 1.2 \text { DI } & 1.8 E \text { OI } & 6 & 33 & 10.34 & 56.90\end{array}$

HISTGGRAM FOR COLUMN 19 ( NB PPM )

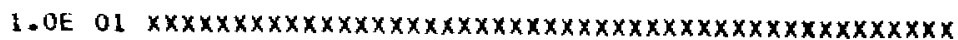

1.5E $01 \times \times \times \times \times \times \times \times \times x$

$\begin{array}{ccccccc}N & L & H & B & T & G & \text { ANALYTICAL } \\ 0 & 25 & 0 & 0 & 0 & 6 & \text { VALUES } \\ 0.0 & 43.10 & & & 0.0 & 0.0 & 33\end{array}$

MAXIMUM $=1.50000 E 01$

MINIMUM $=1,00000 \mathrm{E}$ 01

GEDMETRIC MEAN $=1.07649 E$ OI

GEOMETRIC DEVIATION $=1.17226 E$ OO 


\begin{tabular}{|c|c|c|c|}
\hline \multicolumn{4}{|c|}{ LIMI } \\
\hline & $M E R$ & & $P E R$ \\
\hline $.8 \mathrm{E}$ & 00 & - & $5.6 E$ \\
\hline $.6 \mathrm{E}$ & 00 & - & R. $3 \mathrm{E}$ \\
\hline $.3 E$ & 00 & - & $1.2 \mathrm{E}$ \\
\hline $.2 \mathrm{E}$ & 01 & - & $1.8 \mathrm{E}$ \\
\hline l. $\theta t$ & 01 & - & $2.6 \mathrm{E}$ \\
\hline $2.6 E$ & 01 & - & $3.8 \mathrm{E}$ \\
\hline $3.8 \mathrm{E}$ & 01 & - & $5.6 \mathrm{E}$ \\
\hline $.6 \mathrm{E}$ & DI & - & $8.3 E$ \\
\hline $.3 E$ & 01 & - & $1.2 E$ \\
\hline $2 \mathrm{E}$ & 02 & - & \\
\hline
\end{tabular}

$\begin{array}{rr}\text { FRE } & \text { FREO } \\ 2 & \\ 5 & \\ 5 & 1 \\ 4 & 16 \\ 9 & 25 \\ 15 & 40 \\ 3 & 43 \\ 4 & 47 \\ 8 & 55 \\ 1 & 56\end{array}$

$\begin{array}{ccr}\text { FEE } & \text { PERCENT } & \text { PERCENT } \\ \text { CUM } & \text { FREQ } & \text { FREQ CUM } \\ 2 & 3.45 & 3.45 \\ 7 & 8.62 & 12.07 \\ 12 & 8.62 & 20.69 \\ 16 & 6.90 & 27.59 \\ 25 & 15.52 & 43.10 \\ 40 & 25.86 & 66.97 \\ 43 & 5.17 & 74.14 \\ 47 & 6.90 & 81.03 \\ 55 & 13.79 & 94.83 \\ 56 & 1.72 & 96.55\end{array}$

HISTOGRAM FUR COLUMA

20 NI PPM)

$5.0 t 00 x \times x$

7. OE OO $x \times x \times x \times x \times x$

1.0E $01 \times \times \times \times \times \times \times \times x$

1. $5 E$ ol $x \times \times x \times x \times$

2.0E Ol $x \times x \times x \times x \times x \times x \times x x x \times x$

3.0E $01 \quad x \times x \times x \times x \times x \times x \times x \times x \times x \times x \times x \times x \times x \times x$

5.0E $01 \times \times \times \times \times$

$\omega$

7.0E O1 $x \times \times \times \times \times x$

1.0E $02 \quad x \times \times \times \times \times \times \times \times x \times x \times x$

$1.5 \mathrm{E} 02 \mathrm{xx}$

$\begin{array}{ccccccc}N & L & H & B & T & G & \text { ANALYTICAL } \\ 2 & 0 & 0 & 0 & 0 & 0 & 56 \\ 3.45 & 0.0 & & & 0.0 & 0.0 & \end{array}$

MAXIMU:A $=1.50000 E 02$

MINIMUM $=5.00000 E 00$

GEOMETRIC MEAN $=2.665 \mathrm{H} 6 \mathrm{E}$ OI

GEOMEIRIC DEVIATION $=2.43375 E$ OO 


\begin{tabular}{|c|c|c|c|c|c|c|c|c|}
\hline \multicolumn{5}{|c|}{ LIMITS } & FREO & $\begin{array}{r}\text { FREQ } \\
\text { CUM }\end{array}$ & $\begin{array}{c}\text { PERCENT } \\
\text { FREQ }\end{array}$ & $\begin{array}{l}\text { PERCENT } \\
\text { FREQ CUM }\end{array}$ \\
\hline $8.3 \mathrm{E}$ & ou & - & I. $2 \mathrm{E}$ & 01 & 5 & 5 & 8.62 & 8.62 \\
\hline $1.2 \mathrm{E}$ & 0.1 & - & $1 . \mathrm{BE}$ & 01 & 4 & 9 & 6.90 & 15.52 \\
\hline $1 . b E$ & 01 & - & $2.6 \mathrm{E}$ & 01 & 3 & 12 & 5.17 & 0.69 \\
\hline $2.6 \mathrm{E}$ & 01 & - & $3.8 \mathrm{E}$ & 01 & 1 & 13 & 1.72 & 22.41 \\
\hline $3.8 E$ & 01 & - & $5.6 \mathrm{E}$ & 01 & 0 & 13 & 0.0 & 22.41 \\
\hline $5.6 \mathrm{E}$ & 01 & - & B. $3 E$ & al & 0 & 13 & 0.0 & 22.41 \\
\hline $8.3 \mathrm{E}$ & 01 & - & I. $.2 \mathrm{E}$ & 02 & 0 & 13 & 0.0 & 22.41 \\
\hline $.2 \mathrm{E}$ & 02 & - & $1.8 \mathrm{E}$ & 02 & 1 & 14 & 1.72 & 24.14 \\
\hline
\end{tabular}

HISTOGRAM FOR COLUMN 21 ( PB PPM

1. OE OL $\times \times \times \times \times \times \times \times x$

1.5E $01 \times x \times \times \times x \times$

2. OE O1 $\times \times \times \times \times$

$3.0 t 01 \mathrm{xx}$

$5.0 E 01$

$7.0 E \quad 01$

$1.0 E \quad 02$

1.5E $02 \times x$

$\ddot{\circ}$

$\begin{array}{cc}N & L \\ 31.72 & 14 \\ & 24.14\end{array}$

MAXIMUM $=1.50000 E 02$

HINIMUM $=1.00000 E$ OI

GEOMETRIC MEAIV $=1.70963$ O Ol

GEOMETRIC OEVIATIUN $=2.04059$ E 00
ANALYTICAL

VALUES

$\begin{array}{ccc}T & G & \text { ANALYIICAL } \\ \text { VALUES } \\ 0 & 0 & 14 \\ 0.0 & 0.0 & \end{array}$


FREQUENCY TABLE FOR COLUMN 22 , SB PPM )

\begin{tabular}{|c|c|c|c|c|c|c|}
\hline LOWER & $\begin{array}{l}\text { S } \\
\text { PPER }\end{array}$ & FREQ & $\begin{array}{l}\text { FREQ } \\
\text { CUM }\end{array}$ & $\begin{array}{l}\text { PERCENT } \\
\text { FRER }\end{array}$ & $\begin{array}{l}\text { PERCENT } \\
\text { FREO CUM }\end{array}$ & \\
\hline$N$ & L & H & B & $\mathrm{T}$ & G & $\begin{array}{l}\text { ANALYTICAL } \\
\text { VALUES }\end{array}$ \\
\hline 8 & 0 & 0 & 0 & 0 & 0 & 0 \\
\hline$* * *$ & 0.0 & & & 0.0 & 0.0 & \\
\hline
\end{tabular}

MAXIMUM $=-9.99900 E 48$

MINIMUM $=9.99900 E 48$

GEGMETRIC MEAN $=9.99900$ E $4 \mathrm{~B}$

GEOMETRIC DEVIATIOH $=9.49900 E 48$ 
FREQUENCY TABLE FOR COLUMA 23 I SC PPM I

\begin{tabular}{|c|c|c|c|c|c|c|c|c|}
\hline \multicolumn{5}{|c|}{ LIMITS } & FREQ & FREQ & PERCENT & PERCENT \\
\hline $3.8 \mathrm{E}$ & 00 & - & $5.6 \mathrm{~F}$ & 00 & 0 & CUM & FREQ & FREQ CUM \\
\hline $6 \mathrm{E}$ & 00 & - & A. $3 \mathrm{E}$ & 00 & 5 & 13 & 13.32 & 15.52 \\
\hline $8.3 \mathrm{E}$ & 00 & - & $1.2 \mathrm{E}$ & 01 & 6 & 20 & 10.34 & 3408 \\
\hline $1.2 \mathrm{E}$ & 01 & - & I. BE & 01 & 15 & 35 & 25.86 & 60.34 \\
\hline $1.3 E$ & 01 & - & $2.6 \mathrm{E}$ & 01 & 7 & 42 & 12.07 & 72.41 \\
\hline $2.6 \mathrm{E}$ & 01 & - & 3. $8 \mathrm{E}$ & 01 & B & 48 & 10.34 & 82.76 \\
\hline $3 . \mathrm{dE}$ & 01 & - & $5.6 E$ & 01 & 1 & 49 & 1.72 & 84.48 \\
\hline
\end{tabular}

HISTCGRAM FOR COLUMN 23 \& SC PPM ।

5.0E $00 \quad \times \times \times \times \times \times \times \times \times \times x \times x \times x \times$

7. OE $00 \quad \times \times \times \times \times \times \times \times x$

1. OE $01 \quad x \times x \times x \times x \times x \times$

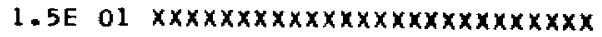

$2.0 E \quad 01 \times \times \times \times \times \times \times \times \times \times \times x$

3. OE OI $x \times x \times x \times x \times x \times$

5. OE $01 \times x$

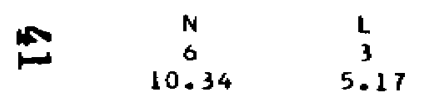

MAXIMUM $=5.00000 E$ OI

MINIMUM $=5.00000$ E 00

GEOMETRIC MEAN $=1.25457 E$ OI

GEOMETRIC DEVIATION $=1.84803$ E 00 
FKEQUENCY TABLE FUR COLUMN 24 I SN PPM ,

\begin{tabular}{|c|c|c|c|c|c|c|c|}
\hline \multicolumn{4}{|c|}{ LIMI IS } & FREQ & FREO & PERCENT & PERCENT \\
\hline LOHE & - & UPPER & & & CUM & FREQ & FREQ CUM \\
\hline B.3E 0 & - & $1.2 \mathrm{E}$ & 01 & 0 & 0 & 0.0 & 0.0 \\
\hline $1.2 \mathrm{E}$ & - & $1.8 \mathrm{E}$ & ol & 0 & 0 & 0.0 & 0.0 \\
\hline $1.8 E$ & - & $2.6 \mathrm{E}$ & 01 & 1 & 1 & 1.72 & 1.72 \\
\hline $2.6 \mathrm{E}$ & - & $3.8 \mathrm{E}$ & 01 & 1 & 2 & 1.72 & 3.45 \\
\hline
\end{tabular}

HISTOGRAM FOR CULUMTE 24 I SN PPM ,

2. OE OI $X X$

3. OE OL XX

$\begin{array}{ccccccc}N & L & H & & & & \text { ANALYTICAL } \\ 56 & 0 & 0 & 0 & 0 & 0 & 2 \\ 96.55 & 0.0 & & & 0.0 & 0.0 & \end{array}$

MAXIMUM $=3.00000 E 01$

MINIMUM $=2.00000 E \mathrm{OL}$

GEOMEIRIC MEAN $=2.4494 \mathrm{BE}$ OI

GEOMEIRIC DEVIATION = $1.33203 E 00$ 
FREQUENCY TAELE FUR COLUMH

25 ISR PPM I

\begin{tabular}{|c|c|c|c|c|c|c|c|}
\hline \multicolumn{4}{|c|}{ LIMIIS } & FREQ & $\begin{array}{l}\text { FREQ } \\
\text { CUM }\end{array}$ & $\begin{array}{l}\text { PERCENI } \\
\text { FREO }\end{array}$ & $\begin{array}{l}\text { PERCENT } \\
\text { FREO CUM }\end{array}$ \\
\hline JE & 01 & $-\quad 1.2 \mathrm{E}$ & 02 & 5 & 5 & 8.62 & 8.62 \\
\hline $2 E$ & 02 & $1.8 \mathrm{E}$ & 02 & 5 & 10 & 8.62 & 17.24 \\
\hline $1.8 E$ & 02 & $2.6 \mathrm{E}$ & 02 & 6 & 16 & 10.34 & 27.59 \\
\hline $2.6 E$ & 02 & $3.8 \mathrm{E}$ & 02 & 18 & 34 & 31.03 & 58.62 \\
\hline 3. $8 E$ & 02 & $5.6 E$ & 02 & 8 & 42 & 13.79 & 72.41 \\
\hline $.6 E$ & 02 & $8.3 E$ & 02 & 4 & 46 & 6.90 & 79.31 \\
\hline
\end{tabular}

HISTUGRAM FUR CULUMN 25 I SR PPM ,

1. OE $02 \times \times \times \times \times \times \times \times x$

1. SE $02 \quad x \times x \times x \times x \times x$

2. OE $02 \times \times \times \times \times \times \times \times \times x$

3. 0 OE $\quad x \times x \times x \times x \times x \times x \times x \times x \times x \times x \times x \times x \times x \times x \times x \times x$

5. OE $02 \quad x \times \times \times \times \times \times \times \times \times \times \times \times x$

7. OE $02 \times \times \times \times \times \times x$

$\begin{array}{ccccccc}\text { N } & \text { L } & H & & & & \text { ANALYTICAL } \\ 9 & 3 & 0 & B & T & G & \text { VALUES } \\ 15.52 & 5.17 & & 0 & 0 & 0 & 46\end{array}$

MAXIMUM $=7.00000 E$ O2

MINIMUM $=1.00000 E 02$

GEOMETRIC MEAN $=2.75514 \mathrm{E} 02$

GEOHETRIC DEVIATION = 1.74864 EO 
FREQUENCY TAPLE FOR COLUMN

26 $\{V P P M]$

\begin{tabular}{|c|c|c|c|c|c|c|c|c|}
\hline & \multicolumn{2}{|c|}{ LIMIIS } & & & FREQ & $\begin{array}{l}\text { FREQ } \\
\text { CUM }\end{array}$ & $\begin{array}{c}\text { PERCENT } \\
\text { FREQ }\end{array}$ & $\begin{array}{l}\text { PERCENT } \\
\text { FREQ CUM }\end{array}$ \\
\hline 8. $3 \mathrm{E}$ & 00 & - & $1.2 E$ & 01 & 0 & 0 & 0.0 & 0.0 \\
\hline $1.2 \mathrm{E}$ & 01 & - & $1.8 E$ & 01 & 5 & 5 & ค. 62 & 8.62 \\
\hline $1 . d t$ & 01 & - & $2.6 \mathrm{E}$ & 01 & 3 & $\theta$ & 5.17 & 13.79 \\
\hline $2.6 \mathrm{t}$ & 01 & - & $3.8 \mathrm{E}$ & 01 & 5 & 13 & 8.62 & 22.41 \\
\hline $3.6 E$ & ol & - & $5.6 \mathrm{E}$ & 01 & 2 & 15 & 3.45 & 25.86 \\
\hline $5.6 \mathrm{E}$ & 01 & - & B. $3 E$ & 01 & 6 & 21 & 10.34 & 36.21 \\
\hline B. $3 t$ & 01 & - & $1.2 \mathrm{E}$ & 02 & 4 & 25 & 6.90 & 43.10 \\
\hline $1.2 \mathrm{E}$ & 02 & - & $1.8 \mathrm{E}$ & 02 & 13 & 38 & 22.41 & 65.52 \\
\hline $1.8 E$ & 02 & - & $2.6 E$ & 02 & 5 & 43 & E.62 & 74.14 \\
\hline $2.6 E$ & 02 & - & $3.8 \mathrm{E}$ & 02 & 10 & 53 & 17.24 & 91.38 \\
\hline $3.8 \mathrm{t}$ & 02 & - & $5.6 \mathrm{t}$ & 02 & 3 & 56 & 5.17 & 96.55 \\
\hline $5.6 E$ & 02 & - & B. 3E & 02 & 2 & 58 & 3.45 & 100.00 \\
\hline
\end{tabular}

HISTOGRAM FOR COLUMM 26 I V PPM

1.5E $01 \times \times \times \times \times \times \times \times \times$

2. Ot Ol $\times \times \times \times x$

3 . Ot of $\times \times \times \times \times \times \times \times \times x$

$5.0501 \times \times x$

7. OE $01 \times \times \times \times \times \times \times \times \times \times$

1. OE $02 \quad \times \times \times \times \times \times x$

1. 5t $02 \quad \times \times \times \times \times \times \times \times \times \times \times \times \times \times \times \times \times \times \times \times \times x$

2.0E $02 \times \times \times \times \times \times \times \times x$

3.0E $02 \times x \times x \times x \times x \times x \times x \times x \times x \times$

5. 0 E $02 \times \times \times \times \times$

7.0E $02 \times \times x$

$\begin{array}{ccccccc}N & L & H & B & T & 0 & \text { AHALYT JCAL } \\ 0 & 0 & 0 & 0 & 0 & 0 & 58 \\ 0.0 & 0.0 & & & 0.0 & 0.0 & \end{array}$

MAXIMUM $=7.0000$ UE 02

MINIMUM $=1.50000$ E 01

GEUMETRIC MEAN = $1.04221 E 02$

GEOMEIRIC DEVIATION $=2.94107$ OO 
Frequency table fur collumn

27 \{ PPM ।

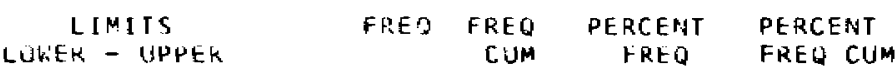

$\begin{array}{cc}1 . & \mathrm{L} \\ 50 & 0 \\ * \neq * 4 & 0.0\end{array}$

8
0

MAX[MUM $=-9.99900 t 49$

MINIMUM $=9.99900 E 48$

GECMETRIC MEAN $=9.99900 E 4 \mathrm{~d}$

GEOMETRIC DEVIATION $=9.99900 E 48$ 
FREGGEVCY TAZLE FUR CULUMTS 28 ( Y PPM )

\begin{tabular}{|c|c|c|c|c|c|c|c|}
\hline \multicolumn{4}{|c|}{ LIMITS } & FHEQ & FKEO & PERCENT & PERCENT \\
\hline LOWE. & - & UPDER & & & CUM & FREO & FREQ CUM \\
\hline 8.310 & $1-$ & $1.2 E$ & 01 & 19 & 19 & 32.76 & 32.76 \\
\hline $1.2 \mathrm{E}$ & - & $1.8 E$ & 01 & 9 & 2.8 & 15.52 & 48.28 \\
\hline $1 .+H t$ & - & $2.6 E$ & 01 & 16 & 44 & 27.59 & 75.86 \\
\hline $2.61=$ & - & $3.8 \mathrm{E}$ & OI & 7 & 51 & 12.07 & 87.93 \\
\hline
\end{tabular}

HISTISGRAM FOR CILUMN $28:$ Y PPM,

1.0t $01 \quad x \times x \times x \times x \times x \times x \times x \times x \times x \times x \times x \times x \times x \times x \times x \times x \times x \times x \times x$

1.5t ol $x \times x \times x \times x \times x \times x \times x \times x \times$

$2.0 \mathrm{E}$ O1 $x \times x \times x \times x \times x \times x \times x \times x \times x \times x \times x \times x \times x \times x x$

3.0E $01 \times x \times x \times x \times x \times x \times x \times$

$\begin{array}{ccccccc}N & \text { L } & H & & & & \text { ANALYIICAL } \\ 2 & 5 & 0 & 0 & 0 & 0 & \text { VALUES } \\ 3.45 & 8.62 & & 0 & 0 & 0 & 51\end{array}$

MAXIMUM $=3.00$ COUE OL

MINIMUM $=1.00000 E$ Ol

GLOMETRIC MEAIV $=1.55237 \mathrm{E}$ O1

GEOMETRIC DEVIATION $=1.48258 E$ OO 
FRENUENCY TAHLE FUR CDLUMN $2912 N$ PPM ,

\begin{tabular}{|c|c|c|c|c|c|c|c|}
\hline \multicolumn{4}{|c|}{ LIMITS } & \multirow[t]{2}{*}{ FREQ } & \multirow{2}{*}{$\begin{array}{l}\text { FREO } \\
\text { CUM }\end{array}$} & \multirow{2}{*}{$\begin{array}{c}\text { PERCENT } \\
\text { FPEQ }\end{array}$} & \multirow{2}{*}{$\begin{array}{l}\text { PERCENT } \\
\text { FREO CUM }\end{array}$} \\
\hline & NER & - UPPER & & & & & \\
\hline $1 . \partial E$ & 02 & $2.6 E$ & 02 & 2 & 2 & 3.45 & 3.45 \\
\hline $2.6 t$ & 02 & $3.8 \mathrm{E}$ & 02 & 3 & 5 & 5.17 & 6.62 \\
\hline $3.0 \mathrm{t}$ & 02 & $5.6 \mathrm{E}$ & 02 & 0 & 5 & 0.0 & 8.62 \\
\hline $5.6 \mathrm{E}$ & 02 & H. $3 E$ & 02 & 3 & B & 5.17 & 13.79 \\
\hline
\end{tabular}

HISTOGRAM FIIR COLUMN 29 I LN PPM

$2.0 E \quad 02 \times \times \times$

3. OE O2 $\times \times \times \times \times$

$5.0 \mathrm{t} 02$

7. Ot $02 \times \times \times \times x$

\begin{tabular}{|c|c|c|c|c|c|c|}
\hline & & & & & & ANALYT ICAL \\
\hline 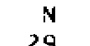 & $\mathbf{L}$ & H & $B$ & T & G & VALUES \\
\hline 29 & $\begin{array}{c}21 \\
30.21\end{array}$ & 0 & 0 & 0 & 0.0 & \\
\hline
\end{tabular}

MAXIMUM $=7.00000 E 02$

MINIMUM $=2.00000$ E 2

GEOMETRIC MEAN $=3.72466502$

GEUMETRIC DEVIAIION $=1.73121 \mathrm{E}$ 00

少 


\begin{tabular}{|c|c|c|c|c|}
\hline \multicolumn{5}{|c|}{ LIMITS } \\
\hline LO & FK & - & PEK & \\
\hline 9. $3 E$ & 00 & - & $1.2 \mathrm{~F}$ & ul \\
\hline $1.2 E$ & 01 & - & $1.8 E$ & 01 \\
\hline $1.8 E$ & 01 & - & $2.6 \mathrm{E}$ & 01 \\
\hline $2.6 \mathrm{E}$ & 01 & - & $3.4 \mathrm{E}$ & 01 \\
\hline 3. $\Delta E$ & 01 & & $5.6 \mathrm{~F}$ & 01 \\
\hline $5.6 \bar{E}$ & 01 & - & $8.3 E$ & 01 \\
\hline $8.3 E$ & 01 & - & $1.2 E$ & 02 \\
\hline $1.2 \mathrm{E}$ & 02 & - & $1.8 \mathrm{E}$ & 02 \\
\hline $1.8 E$ & 02 & - & $2.6 \mathrm{E}$ & 02 \\
\hline $2.6 E$ & 02 & & $3 . e E$ & 02 \\
\hline $3.8 \mathrm{E}$ & 02 & - & $5.6 \mathrm{E}$ & 02 \\
\hline
\end{tabular}

$\begin{array}{cr}\text { FREG } & \text { FRE } \\ & \text { CUM } \\ 0 & 0 \\ 0 & 0 \\ 0 & 0 \\ 1 & 1 \\ 7 & 8 \\ 31 & 39 \\ 8 & 47 \\ 3 & 50 \\ 2 & 52 \\ 0 & 52 \\ 1 & 33\end{array}$

$\begin{array}{cc}\text { PERCENT } & \text { PERCENT } \\ \text { FREQ } & \text { FREQ CUM } \\ U .0 & 0.0 \\ 0.0 & 0.0 \\ 0.0 & 0.0 \\ 1.72 & 1.72 \\ 12.07 & 13.79 \\ 53.45 & 67.24 \\ 13.79 & 81.03 \\ 5.17 & 86.21 \\ 3.45 & 89.66 \\ 0.0 & 89.66\end{array}$

HISTOGRAM FOR COLUMN

$3012 R$ PPM

3. OE OI $x \times$

5.0t $01 \quad \times x \times x \times \times \times \times \times \times \times \times x$

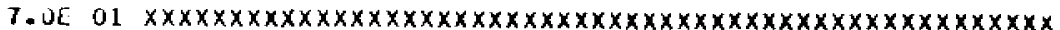

1.OE $02 \times \times \times \times \times \times \times \times \times \times \times \times \times \times$

1.5E $02 \times \times \times \times x$

$2.0 \mathrm{E} 02 \times \times \times$

3. UE: 02

$\mathbf{0}$

5.0E $02 \times x$

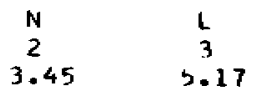

B

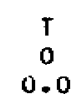

6
0
0.0

ANALYTICAL

VALUES

MAXIMUN $=5.00000 E 02$

MINIMUM $=3.00000 E$ OI

GEOMEIKIC MEAN $=7.83938 \mathrm{E}$ OI

GEOMEIRIC DEVIATION $=1.53892 \mathrm{E}$ OO 


\begin{tabular}{|c|c|c|}
\hline \multicolumn{3}{|c|}{ LIMITS } \\
\hline LOWER & & PER \\
\hline 1. $\triangle E-02$ & - & $2 \cdot 6 E-32$ \\
\hline $2.6 \mathrm{E}-02$ & - & 3. $8 \mathrm{E}-02$ \\
\hline 1. $B E-02$ & - & $5.6 \mathrm{E}-02$ \\
\hline $5.6 E-02$ & - & 9. $3 E-02$ \\
\hline B. $3 E-02$ & - & $1.2 E-01$ \\
\hline $1.2 \mathrm{E}-01$ & - & 1. $\Delta E-01$ \\
\hline $1 \cdot B E-01$ & - & $2.6 t-01$ \\
\hline $2.6 \mathrm{E}-01$ & - & 3. $d E-U 1$ \\
\hline $3.8 \mathrm{E}-0 \mathrm{I}$ & & $5.6 \mathrm{E}-01$ \\
\hline $5.6 t-01$ & - & B. $3 \mathrm{E}-0 \mathrm{I}$ \\
\hline $\mathcal{H} \cdot 3 E-01$ & & $1.2 \mathrm{t} 0 \mathrm{O}$ \\
\hline $1.2 \mathrm{E} 00$ & & 1. $B E D U$ \\
\hline
\end{tabular}

$\begin{array}{cc}\text { FREO } & \text { FR } \\ & \\ 0 & \\ 0 & \\ 0 & \\ 1 & \\ 0 & \\ 0 & \\ 0 & \\ 0 & \\ 0 & \\ 0 & \\ 0 & \\ 1 & \end{array}$

PERTENT

3. $8 \mathrm{E}-02$

$5.6 E-02$

CUM FREO

PERCENT
FREU CUM

$0.0 \quad 0.0$

0.0

1.72

$0.0 \quad 1.72$

$0.0 \quad 1.72$

$0.0 \quad 1.72$

$0.0 \quad 1.72$

$0.0 \quad 1.72$

$0.0 \quad 1.72$

$\begin{array}{ll}0.0 & 1.72 \\ 1.72 & 3.45\end{array}$

HISTOGRAM FOR COLUMN 31 ( AU PPM)

7. OE $-02 \times x$

$1.0 \mathrm{E}-01$

$1.5 t-01$

$2.0 E-0.1$

3. OE-OI

5. OE-Ol

$\infty$

$7.0 E-01$

$1 . O E O O$

1. $5 E$ DO $\times x$

\begin{tabular}{|c|c|c|c|c|c|c|}
\hline$N$ & L & $\mathrm{H}$ & B & $\mathbf{T}$ & $\mathbf{G}$ & $\begin{array}{l}\text { ANALYT ICAL } \\
\text { VALUES }\end{array}$ \\
\hline $\begin{array}{c}0 \\
0.0\end{array}$ & $\begin{array}{l}36 \\
96.55\end{array}$ & 0 & 0 & $\stackrel{0}{0.0}$ & $\begin{array}{c}0 \\
0.0\end{array}$ & 2 \\
\hline
\end{tabular}

MAXIMUM $=1.50000 E$ OD

MINIMUM $=6.00000 E-02$

GEONETRIC MEAN $=3.000 U L-01$

GEOMETRIC UEVIATION $=9.73851$ EOO 


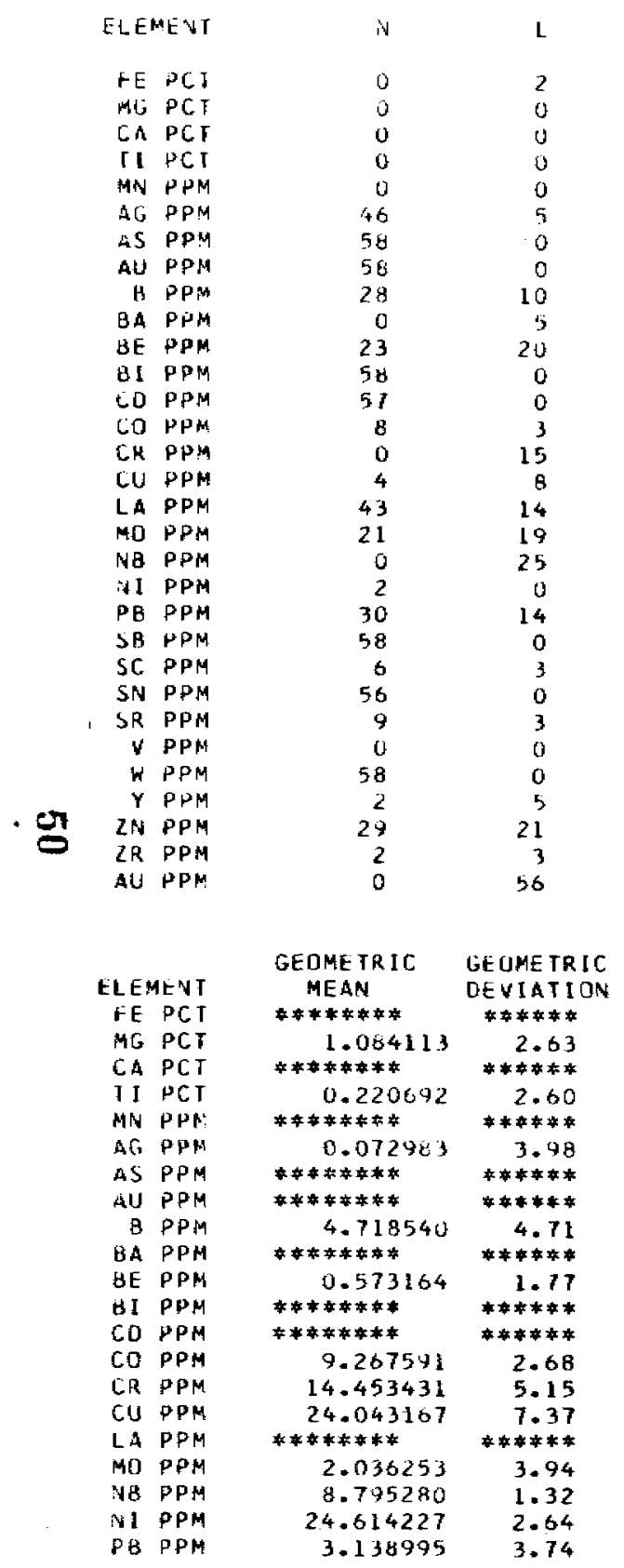

\begin{tabular}{|c|c|c|c|c|}
\hline$H$ & B & $T$ & $G$ & $\begin{array}{l}\text { ANAL Y I I CA } \\
\text { VALUES }\end{array}$ \\
\hline 0 & 0 & 0 & 1 & 55 \\
\hline 0 & 0 & 0 & 0 & 58 \\
\hline 0 & 0 & 0 & 2 & 56 \\
\hline 0 & 0 & 0 & 0 & 58 \\
\hline 0 & 0 & 0 & 2 & 56 \\
\hline 0 & 0 & 0 & 0 & 7 \\
\hline 0 & 0 & 0 & 0 & 0 \\
\hline 0 & 0 & 0 & 0 & 0 \\
\hline 0 & 0 & 0 & 0 & 20 \\
\hline 0 & 0 & 0 & 1 & 52 \\
\hline 0 & 0 & 0 & 0 & 15 \\
\hline 0 & 0 & 0 & 0 & 0 \\
\hline 0 & 0 & 0 & 0 & l \\
\hline 0 & 0 & 0 & 0 & 47 \\
\hline 0 & 0 & 0 & 0 & 43 \\
\hline 0 & 0 & 0 & 0 & 46 \\
\hline 0 & 0 & 0 & 0 & 1 \\
\hline 0 & 0 & 0 & 0 & 18 \\
\hline 0 & 0 & 0 & 0 & 33 \\
\hline 0 & 0 & 0 & 0 & 56 \\
\hline 0 & 0 & 0 & 0 & 14 \\
\hline 0 & 0 & 0 & 0 & 0 \\
\hline 0 & 0 & 0 & 0 & 49 \\
\hline 0 & 0 & 0 & 0 & 2 \\
\hline 0 & 0 & 0 & 0 & 46 \\
\hline 0 & 0 & 0 & 0 & 58 \\
\hline 0 & 0 & 0 & 0 & 0 \\
\hline 0 & 0 & 0 & 0 & 51 \\
\hline 0 & 0 & 0 & 0 & 8 \\
\hline 0 & 0 & 0 & 0 & 53 \\
\hline 0 & 0 & 0 & 0 & 2 \\
\hline
\end{tabular}

REMARKS

1 greater than Values. no computations.

58 SAMPLES AND 58 ANALYTICAL VALUES.

2 GREATER THAN VALUES. NO COMPUTATIONS.

58 SAMPLES AND 58 ANALYTICAL VALUES.

2 GREATER THAN VALUES. NO COMPUTATIONS.

$5 I$ NOT DEIECTED, LESS THAN, OR TRACE VALUES.
58 NOT DETECTED, LESS IHAN, DR TRACE VALUES.

58 NOT DETECTED, LESS THAN, DR TRACE VALUES.

38 NOT DETECTED, LESS THAN, OR TRACE VALUES.

1 GREATER THAN VALUES. NO COMPUTATIONS.

43 NOT DETECIED, LESS THAN, OR TRACE VALUES.

58 NOT DETECTED, LESS THAN, OR TRACE VALUES.

11 NOT OETECTED, LESS THAN, OR TRACE VALUES

15 NOT DEIECTED, LESS THAN, OR TRACE VALUES

12 NOT DETECTED, LESS THAN, OR TRACE VALUES

57 NOT DETECTED, LESS THAN, OR TRACE VALUES

57 NOT DETECTED, LESS THAN, OR TRACE VALUES.

45 NOT DETECTED, LESS THAN, OR TRACE VALUES.

25 NOT DETECTED, LESS THAN, OR TRACE VALUES.

2 NOT DEIECTED, LESS THAN, CR TRACE VALUES.
44 NOT DETECTED, LESS THAN, OR TRACE VALUES.

7 REPURTED VALUES.

O REPORTEO VALUES. NO COMPUTATIONS.

0 REPORTED VALUES. NO COMPUTATIONS.

20 REPOR TED VALUES.

15 REPORTEO VALUES.

O REPORTED VALUES. NO CDHPUTATIONS.

1 REPORTEO VALUES. NO COMPUTATIONS.

47 REPORTEO VALUES.

43 REPORTED VALUES.

1 REPORTED VALUES. NO COMPUTATIDNS.

18 REPORTED VALUES.

33 REPOR TED VALUES.

33 REPOR TEO VALUES.

14 REPORTED VALUES. 


\begin{tabular}{|c|c|c|c|}
\hline 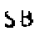 & $\mu P M$ & $4 \div \div \div \div$ & 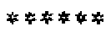 \\
\hline & PPM & 9.794670 & 2.27 \\
\hline 14 & $P P M$ & 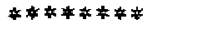 & $\div \div * * *$ \\
\hline & PFM & 195.767548 & 2.34 \\
\hline v & $\mathrm{PH}+\boldsymbol{N}$ & 108.220291 & 2.94 \\
\hline & PPN & 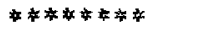 & 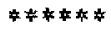 \\
\hline r & ppo & 13.974360 & 1.60 \\
\hline $2 \mathrm{~N}$ & $\mathrm{PP}$ i & 35.951492 & 4.10 \\
\hline & PPM & 63.099716 & 2.27 \\
\hline & $P Q M$ & $* * * * * \#$ & 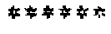 \\
\hline
\end{tabular}

58 NIJ DETECTED, LESS THAN, GR tRACE VALUES.

9 NOT DETECTEO, LESS THAN, OR TRACE VALUES.

56 NOT DETECTEO * LESS THAN, OR TRACE VALUES.

12 NIT DETECTED, LESS THAN, OR TRACE VALUES.

5 \& SAMPLES ANG 58 ANALYTICAL VALUES

50 NIT DETECTED, LESS THAN, OR TRACE VALUES.

7 WOT DETECTED, LESS THAN, UR TRACE VALUES.

50 NUT DETECTEO, LESS THAN, OR TRACE VALUES

3 NOT DETECTEO, LESS THAN, OR TRACE VALUES.

56 NDT DETECTED, LESS THAN, OR TRACE VALUES.
O REPORTEO VALUES. NO COMPUTATIONS.

49 REPORTED VALUES.

2 REPORTED VALUES. NO COMPUTATIONS. 46 REPORTED VALUES.

O REPORTED VALUES. NO COMPUTATIONS. 51 REPORTED VALUES.

8 REPURTEO VALUES.

53 REPORTEO VALUES.

2 REPORTED VALUES. NO COMPUTATIONS. 
TARLE 3 CRAIG B-5 STREAM SEDIMENTS

\begin{tabular}{|c|c|c|c|c|c|c|c|c|}
\hline ximes & SAMPLE & FE $P C F$ & MG PCT & CA PCT & FI PCT & MN PPM & $A G P P$ & PM \\
\hline 33 & $\operatorname{ACL} 330$ & 3.0000 & 1.5000 & 0.7000 & 0.3000 & 1000.0000 & 0.0 & N \\
\hline 34 & $A C L 339$ & 5.0000 & 2.0000 & 0.7000 & 0.5000 & 1500.0000 & 0.0 & $N$ \\
\hline & ACL 307 & 5.0000 & 1.0000 & 1.0000 & 0.3000 & 3000.0000 & 0.0 & N \\
\hline 36 & $A C L 308$ & 3.0000 & 1.5000 & 0.7000 & 0.3000 & 1500.0000 & 0.0 & L \\
\hline 37 & $A C L 341$ & 10.0000 & 3.0000 & 1.5000 & 0.5000 & 2000.0000 & 0.0 & $\mathrm{~N}$ \\
\hline $3 x$ & $A C L 34 \mathrm{U}$ & 5.0000 & 1.5000 & 0.7000 & 0.3000 & 1500.0000 & 0.0 & N \\
\hline 39 & $A C L 342$ & 5.0000 & 2.0000 & 1.5000 & 0.7000 & 1500.0000 & 0.0 & $\mathbf{N}$ \\
\hline$\$$ & $A C L 336$ & 7. 10000 & 1.5000 & 1.5000 & 0.3000 & 1500.0000 & 0.0 & $\mathbf{N}$ \\
\hline 4 & ACL $31 y$ & 1.0000 & 3.0000 & 3.0000 & 0.5000 & 1500.0000 & 0.0 & N \\
\hline 42 & $4 C L 302$ & 1.0000 & 2.0000 & 1.0000 & 0.5000 & 1500.0000 & 0.0 & $\mathbf{N}$ \\
\hline 43 & $A C L 301$ & 3.0000 & 1.5000 & 0.7000 & 0.3000 & 1500.0000 & 0.0 & L \\
\hline 44 & $A C L 350$ & 5.0000 & $t . b 000$ & 0.1000 & 0.3000 & 1500.0000 & 0.0 & N \\
\hline 45 & $A C L 349$ & 3.0000 & 1.5000 & 0.7000 & 0.2000 & 1000.0000 & 0.0 & $\mathbf{N}$ \\
\hline Ac & $A C L 316$ & 7.0000 & 2.0000 & 1.5000 & 0.7000 & 1500.0000 & 0.0 & N \\
\hline 47 & $A C L 317$ & 10.0000 & 2.0000 & 1.5000 & 0.7000 & 1500.0000 & 0.0 & $N$ \\
\hline 47 & $A C L 316$ & 10.0000 & 3.0000 & 1.5000 & 0.7000 & 1500.0000 & 0.0 & N \\
\hline 4 & $A C L 348$ & 15.0000 & 3.0000 & 1.5000 & 0.7000 & 1500.0000 & 0.0 & N \\
\hline 50 & ACL 344 & 15.0000 & 3.0000 & 1.5000 & 0.7000 & 1500.0000 & 0.0 & $\mathrm{~N}$ \\
\hline 57 & $\mathrm{ACL} 47 \mathrm{~B}$ & 10.0000 & 3.0000 & 2.0000 & 0.7000 & 1500.0000 & 0.0 & h \\
\hline .52 & $\mathrm{ACL} 468$ & 15.0000 & 5.0000 & 2.0000 & 1.0000 & 2000.0000 & 0.0 & $\mathrm{~N}$ \\
\hline 53 & $A C L 310$ & 15.0000 & 3.0000 & 1.5000 & 0.7000 & 1500.0000 & 0.0 & $\mathbf{N}$ \\
\hline 54 & ACL 309 & 15.0000 & 3.0000 & 1.5000 & 0.7000 & 2000.0000 & 0.0 & N \\
\hline 55 & $A C L 311$ & 15.0040 & 3.0000 & 2.0000 & 1.0000 & 1500.0000 & 0.0 & $\mathbf{L}$ \\
\hline 56 & ACL 312 & 15.0000 & 3.0000 & 3.0000 & 1.0000 & 2000.0000 & 0.0 & $\mathrm{~N}$ \\
\hline 57 & $A C L 313$ & 15. vovo & 5.0000 & 2.0000 & 1.0000 & 1500.0000 & 0.0 & N \\
\hline 58 & $\operatorname{ACL} 314$ & 5.0000 & 1.5000 & 1.5000 & 0.7000 & 1500.0000 & 0.0 & N \\
\hline $5 \%$ & $\mathrm{ACL} 315$ & 15.0000 & 3.0000 & 2.0000 & 1.0000 & 2000.0000 & 0.0 & $\mathbf{N}$ \\
\hline & $A C L 469$ & 7.0000 & 1.5000 & 0.7000 & 0.5000 & 1500.0000 & 0.0 & L \\
\hline 6 & ACL 479 & 5.0000 & 1.5000 & 1.5000 & 0.3000 & 700.0000 & 0.5000 & \\
\hline 62 & $A C L 481$ & 15.0000 & 3.0000 & 3.0000 & 0.3000 & 1500.0000 & 0.0 & $L$ \\
\hline 63 & $A C L 471$ & 5.0000 & 2.0000 & 2.0000 & 0.3000 & 1000.0000 & 0.0 & $\mathbf{N}$ \\
\hline 64 & $A C L 470$ & 7.0000 & 2.0000 & 2.0000 & 0.5000 & 1000.0000 & 0.0 & N \\
\hline 65 & $A C L 472$ & 7.0000 & 2.0000 & 1.5000 & 0.5000 & 1500.0000 & 0.0 & $\mathbf{L}$ \\
\hline 6 & $A[1473$ & 3.0000 & 1.0000 & 2.0000 & 0.3000 & 3000.0000 & 0.0 & $\mathbf{L}$ \\
\hline 67 & ACL 459 & 3.0000 & 0.3000 & 1.5000 & 0.2000 & 1500.0000 & 0.0 & L \\
\hline 65 & $A C L 480$ & 2.0000 & 0.7000 & 1.5000 & 0.1500 & 1500.0000 & 0.0 & $\mathbf{N}$ \\
\hline 64 & $A C L 4 B Z$ & 5.0000 & 0.7000 & 1.5000 & 0.3000 & 1500.0000 & 0.5000 & \\
\hline 20 & $A C L 483$ & 3.0000 & 1.0000 & 1.5000 & 0.2000 & 1500.0000 & 0.0 & $N$ \\
\hline $\mathbb{7}$ & $A C L 496$ & 7.0000 & 1.0000 & 1.0000 & 0.5000 & 1000.0000 & 0.0 & N \\
\hline 72. & $\mathrm{ACL} 497$ & 5.0000 & 1.5000 & 1.5000 & 0.5000 & 1500.0000 & 0.0 & N \\
\hline 73 & $\operatorname{ACL} 495$ & 5.00000 & 1.5000 & 1.5000 & 0.3000 & 1500.0000 & 0.0 & s \\
\hline
\end{tabular}

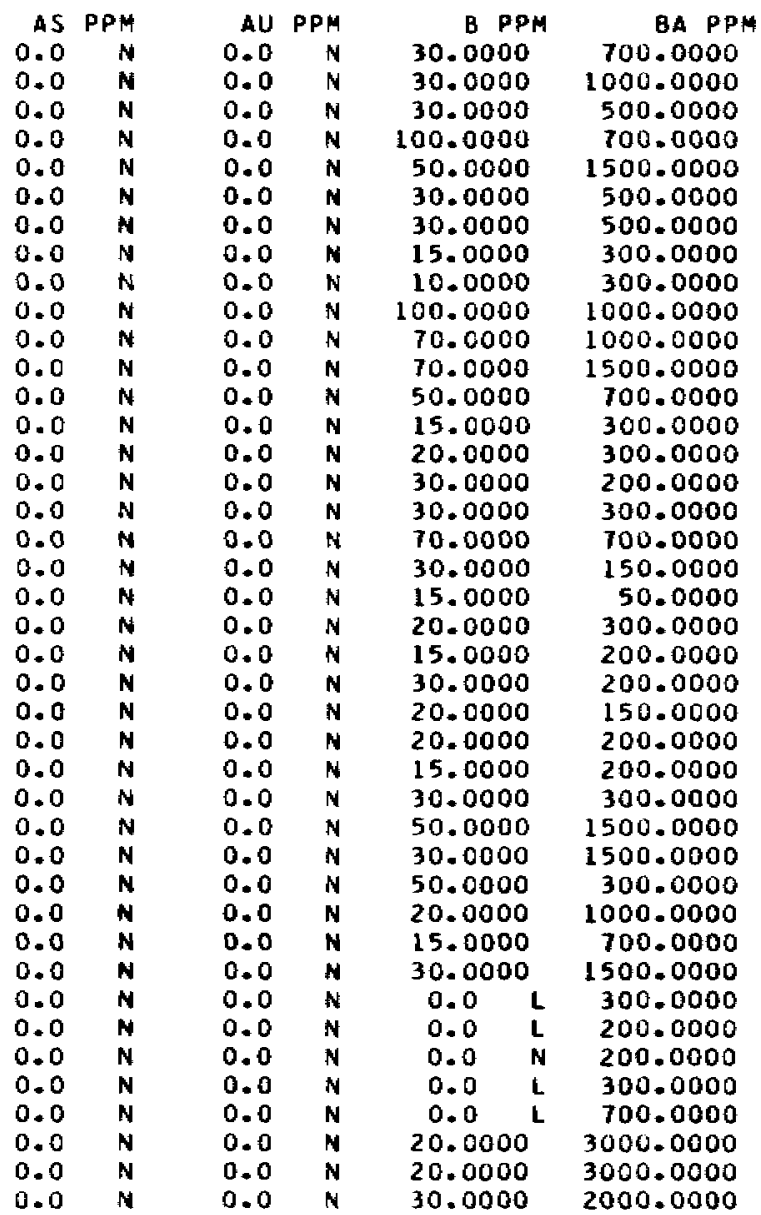


CRAIG B-5 STREAM SEDIMENTS

\begin{tabular}{|c|c|c|c|c|c|}
\hline & SAMPLE & $P Q P P M$ & $S B$ & $P P: A$ & $S C P \rho M$ \\
\hline 33 & $A C L 338^{\circ}$ & 15.0000 & 0.0 & : & 10.0000 \\
\hline 34 & $A C L 334$ & 10.0000 & 0.0 & N & 10.0000 \\
\hline 35 & ACL 307 & 30.0000 & 0.0 & $N$ & 15.0000 \\
\hline 36 & $A C L 30 \mathrm{O}$ & 70.0000 & 0.0 & $\therefore$ & 15.0000 \\
\hline 37 & $A C L 341$ & 20.0000 & 0.0 & $v$ & 20.0000 \\
\hline 38 & $\operatorname{ACL} 340$ & 15.0000 & 0.0 & $\mathrm{~N}$ & 15.0000 \\
\hline 39 & $\mathrm{ACL} 342$ & 10.0000 & 0.0 & v & 15.0000 \\
\hline 40 & $A C L 336$ & 15.0000 & 0.0 & 4 & 15.0000 \\
\hline 4 & $A C L \$ 19$ & 15.0000 & 0.0 & P: & 20.0000 \\
\hline 42 & ACL 302 & 30.0000 & 0.0 & $*$ & 20.0000 \\
\hline 43 & $A C L 301$ & 150.0000 & 0.0 & v & 15.0000 \\
\hline 47 & ACL $\$ S O$ & 150.0000 & 0.0 & $\mathrm{~N}$ & 15.0000 \\
\hline 45 & $\operatorname{ACL} 349$ & 70.0000 & 0.0 & $N$ & 15.0000 \\
\hline 46 & ACL $\$ 16$ & 50.0000 & 0.0 & $\mathbf{N}$ & 30.0000 \\
\hline 47 & $\operatorname{ACL} 317$ & 30.0000 & 0.0 & $\mathrm{~N}$ & 30.0000 \\
\hline 48 & $A C L 3 L$ d & 20.0000 & 0.0 & * & 30.0000 \\
\hline 49 & ACL $34 \mathrm{~d}$ & 10.0000 & 0.0 & $N$ & 30.0000 \\
\hline 50 & $A C L 344$ & 15.0000 & 0.0 & $\mathbf{N}$ & 30.0000 \\
\hline 51 & $A C L 4] 8$ & $0.0 \quad \mathrm{~L}$ & 0.0 & $N$ & 30.0000 \\
\hline 52 & $A C L 468$ & 20.0000 & 0.0 & v & 30.0000 \\
\hline 53 & $A C L 310$ & 15.0000 & 0.0 & $N$ & 30.0000 \\
\hline $5 y$ & $A C L 309$ & 20.0000 & 0.0 & $\mathrm{~N}$ & 30.0000 \\
\hline 55 & $A C L 311$ & 30.0000 & 0.0 & $N$ & 30.0000 \\
\hline 56 & $A C L 312$ & 30.0000 & 0.0 & $\mathrm{~N}$ & 30.0000 \\
\hline 5 & $A C L 313$ & 15.0000 & 0.0 & $*$ & 30.0000 \\
\hline 58 & $A C L 314$ & 15.0000 & 0.0 & $\mathrm{~N}$ & 20.0000 \\
\hline 54 & $A C L 315$ & 15.0000 & 0.0 & $\mathrm{~N}$ & 30.0000 \\
\hline & ACL 469 & 70.0000 & 0.0 & $N$ & 15.0000 \\
\hline & ACL 479 & 15.0000 & 0.0 & N & 10.0000 \\
\hline 62 & $A C 1481$ & 15.0000 & 0.0 & in & 30.0000 \\
\hline 63 & $\mathrm{ACL} 471$ & 20.0000 & 0.0 & $\mathbf{N}$ & 15.0000 \\
\hline 64 & $\triangle C L 470$ & 20.0000 & 0.0 & $\mathbf{N}$ & 15.0000 \\
\hline 65 & ACL 472 & 20.0000 & 0.0 & $N$ & 20.0000 \\
\hline 66 & $A C L 473$ & 15.0000 & 0.0 & $*$ & 7.0000 \\
\hline 67 & ACL 459 & $0.0 \quad 1$ & 0.0 & $v$ & $0.0 \quad \mathrm{~L}$ \\
\hline 6 & $\triangle C L 480$ & 10.0000 & 0.0 & $\mathbf{N}$ & $0.0 \quad \mathrm{~L}$ \\
\hline 6 & $\mathrm{ACL} 4 \mathrm{BZ}$ & 100.0000 & 0.0 & N & 5.0000 \\
\hline 70 & $A C L 483$ & 10.0000 & 0.0 & $N$ & 7.0000 \\
\hline " & $A C L 496$ & 15.0000 & 0.0 & N & 15.0000 \\
\hline $7_{2}$ & ACL 497 & 15.0000 & 0.0 & N & 15.0000 \\
\hline 73 & ACL 495 & 20.0000 & 0.0 & $\mathrm{~N}$ & 15.0000 \\
\hline
\end{tabular}

\begin{tabular}{|c|c|c|c|c|c|}
\hline SN PP & PPM & SR PPM & $V P P M$ & $w$ & PPM \\
\hline 0.0 & $\mathbf{N}$ & 300.0000 & 150.0000 & 0.0 & $N$ \\
\hline 0.0 & $N$ & 300.0000 & 200.0000 & 0.0 & \\
\hline 10.0000 & & 300.0000 & 150.0000 & 0.0 & \\
\hline 100.0000 & & 300.0000 & 200.0000 & 0.0 & \\
\hline 0.0 & $L$ & 500.0000 & 300.0000 & 0.0 & \\
\hline 0.0 & $\mathbf{N}$ & 300.0000 & 200.0000 & 0.0 & \\
\hline 0.0 & $\mathbf{N}$ & 500.0000 & 300.0000 & 0.0 & \\
\hline 0.0 & N & 300.0000 & 200.0000 & 0.0 & \\
\hline 0.0 & $\mathbf{N}$ & 500.0000 & 200.0000 & 0.0 & \\
\hline 30.0000 & & 300.0000 & 300.0000 & 0.0 & \\
\hline 70.0000 & & 300.0000 & 300.0000 & 0.0 & \\
\hline 30.0000 & & 300.0000 & 200.0000 & 0.0 & \\
\hline 30.0000 & & 300.0000 & 150.0000 & 0.0 & \\
\hline 15.0000 & & 200.0000 & 300.0000 & 0.0 & \\
\hline 0.0 & $\mathbf{N}$ & 300.0000 & 300.0000 & 0.0 & \\
\hline 20.0000 & & 300.0000 & 300.0000 & 0.0 & \\
\hline 0.0 & $\mathbf{N}$ & 300.0000 & 300.0000 & 0.0 & \\
\hline 0.0 & $\mathbf{N}$ & 300.0000 & 300.0000 & 0.0 & \\
\hline 0.0 & N & 300.0000 & 300.0000 & 0.0 & \\
\hline 0.0 & $\mathbf{N}$ & 300.0000 & 300.0000 & 0.0 & \\
\hline 0.0 & $\mathbf{N}$ & 200.0000 & 300.0000 & 0.0 & \\
\hline 10.0000 & & 300.0000 & 300.0000 & 0.0 & \\
\hline 15.0000 & & 300.0000 & 300.0000 & 0.0 & \\
\hline 15.0000 & & 300.0000 & 300.0000 & 0.0 & \\
\hline 0.0 & L & 300.0000 & 300.0000 & 0.0 & \\
\hline 0.0 & $N$ & 200.0000 & 200.0000 & 0.0 & \\
\hline 0.0 & $N$ & 200.0000 & 300.0000 & 0.0 & \\
\hline 0.0 & N & 300.0000 & 200.0000 & 0.0 & \\
\hline 0.0 & $N$ & 300.0000 & 200.0000 & 0.0 & \\
\hline 0.0 & N & 300.0000 & 200.0000 & 0.0 & \\
\hline 0.0 & $\mathbf{N}$ & 500.0000 & 200.0000 & 0.0 & \\
\hline 0.0 & $\mathbf{N}$ & 500.0000 & 150.0000 & 0.0 & \\
\hline 0.0 & $\mathbf{N}$ & 300.0000 & 300.0000 & 0.0 & \\
\hline 0.0 & N & 500.0000 & 100.0000 & 0.0 & \\
\hline 0.0 & $\mathbf{N}$ & 500.0000 & 30.0000 & 0.0 & \\
\hline 0.0 & $N$ & 700.0000 & 30.0000 & 0.0 & \\
\hline 0.0 & N & 300.0000 & 30.0000 & 0.0 & \\
\hline 0.0 & $\mathbf{N}$ & 500.0000 & 70.0000 & 0.0 & \\
\hline 0.0 & $\mathbf{N}$ & 300.0000 & 300.0000 & 0.0 & \\
\hline 0.0 & $\mathbf{N}$ & 300.0000 & 300.0000 & 0.0 & \\
\hline 0.0 & $\mathbf{N}$ & 300.0000 & 300.0000 & 0.0 & \\
\hline
\end{tabular}

$Y P P M$
10.0000
15.0000
15.0000
15.0000
15.0000
10.0000
20.0000
15.0000
15.0000
15.0000
15.0000
15.0000
10.0000
20.0000
20.0000
20.0000
15.0000
20.0000
30.0000
20.0000
20.0000
20.0000
20.0000
30.0000
30.0000
20.0000
20.0000
15.0000
20.0000
30.0000
15.0000
15.0000
30.0000
15.0000
10.0000
10.0000
15.0000
15.0000
20.0000
20.0000
15.0000

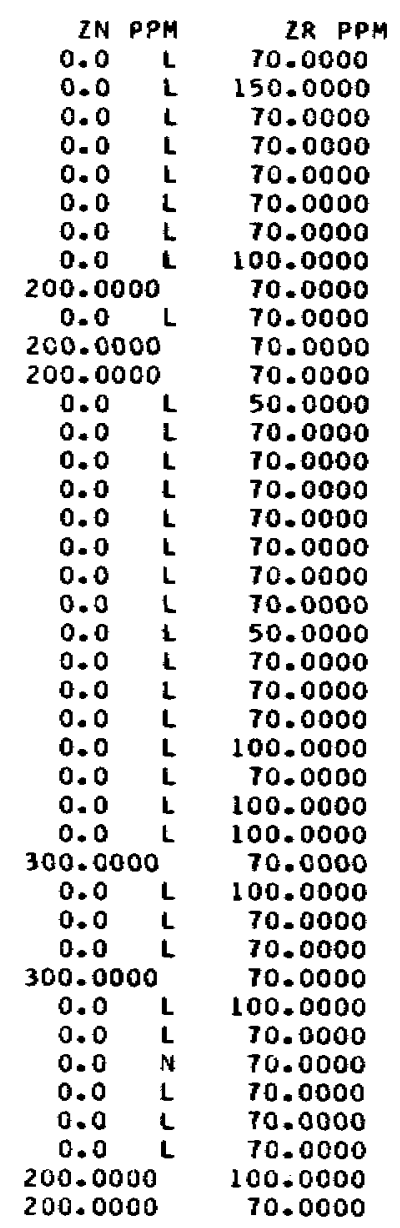


CRAIG B-5 STREAM SEDIMENTS

SAMPLE AU PPM $0.0200 \mathrm{~L}$ ACL 3390.02001 $\mathrm{ACL} 307 \quad 0.1000 \mathrm{~L}$ ACL $308 \quad 0.0200 \mathrm{~L}$ ACL 341 ACL 3410 ACL $3400.0200 \mathrm{~L}$ ACL 342 0.1000 ACL $336 \quad 0.0200 \mathrm{~L}$ ACL 319 0.0200L ACL $302 \quad 0.0400 \mathrm{~L}$ ACL $301 \quad 0.1000 L$ ACL $350 \quad 0.0200 L$ ACL $3490.0200 L$ ACL $316 \quad 0.0200 L$ ACL $317 \quad 0.02004$ ACL 318 ACL 34800202 ACL 344 ACL 344 0.0200L ACL478 ACL468 0.100OL ACL $310 \quad 0.0400 \mathrm{~L}$ ACL $309 \quad 0.0200 \mathrm{~L}$ ACL311 $0.0400 \mathrm{~L}$ ACL $3120.0200 \mathrm{~L}$ ACL $313 \quad 0.0400 \mathrm{~L}$ ACL $314 \quad 0.02004$ ACL $315 \quad 0.1000 \mathrm{~L}$ ACL469 0.02001 ACL 4790.02004 ACL 481 CCL471 $0.0200 \mathrm{~L}$ ACL470 $0.0200 \mathrm{~L}$ ACL470 $0.0200 \mathrm{~L}$ ACL472 $0.0200 L$ ACL $473 \quad 0.1000 \mathrm{~L}$ ACL459 $0.0400 \mathrm{~L}$ ACL480 $\quad 0.0200 \mathrm{~L}$ ACL482 0.3000 $\mathrm{ACL} 483 \quad 0.0200 \mathrm{~L}$ ACL 496 $0.0200 \mathrm{~L}$ ACL497 40.10401 ACL495 


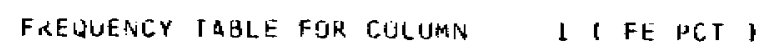

\begin{tabular}{|c|c|c|c|c|c|c|}
\hline L UWER & IM & ER & FREO & $\begin{array}{r}\text { FREQ } \\
\text { CUM }\end{array}$ & $\begin{array}{c}\text { PERCENT } \\
\text { FREO }\end{array}$ & $\begin{array}{l}\text { PERCENT } \\
\text { FREQ CUM }\end{array}$ \\
\hline $3 . \Delta E-02$ & - & 5. $6 E-02$ & 0 & 0 & $\quad 0.0$ & 0.0 \\
\hline $5.6 E-02$ & - & 6. 3E-02 & 0 & 0 & 0.0 & 0.0 \\
\hline $8.3 E-02$ & - & $1.2 t-01$ & 0 & 0 & 0.0 & 0.0 \\
\hline $1.2 \mathrm{E}-0 \mathrm{i}$ & - & $1.8 E-01$ & 0 & 0 & 0.0 & 0.0 \\
\hline $1 . \partial E-01$ & - & $2.6 E-\mathrm{J} 1$ & 0 & 0 & 0.0 & 0.0 \\
\hline $2.6 \mathrm{E}-0 \mathrm{~L}$ & - & 3. $8 \mathrm{E}-01$ & 0 & 0 & 0.0 & 0.0 \\
\hline $3.8 E-01$ & - & $5.6 E-02$ & 0 & 0 & 0.0 & 0.0 \\
\hline $5.6 E-01$ & - & B. $3 E-01$ & 0 & 0 & 0.0 & 0.0 \\
\hline 6. 3E-01 & - & $1.2 \mathrm{E} \quad 00$ & 0 & 0 & 0.0 & 0.0 \\
\hline $1.2 E \quad 00$ & - & $1.8 \mathrm{E} \quad 00$ & 0 & 0 & 0.0 & 0.0 \\
\hline 1.. BE 00 & - & $2.6 \mathrm{E} \quad 00$ & 1 & 1 & 2.44 & 2.44 \\
\hline $2.6 \mathrm{E} 00$ & - & $3.8 E \quad 00$ & $t$ & 8 & 17.07 & 19.51 \\
\hline $3 . \mathrm{dE} \quad 00$ & - & $5.6 \mathrm{E} \quad 00$ & 11 & 19 & 26.63 & 46.34 \\
\hline $5.6 E \quad 00$ & - & $8.3 E \quad 00$ & $\theta$ & 27 & 19.51 & 65.85 \\
\hline B. 3t 00 & - & $1.2 E \quad 01$ & 4 & 31 & 9.76 & 75.61 \\
\hline $1.2 \mathrm{E} \quad 01$ & - & $1.8 \mathrm{E} \quad 01$ & 10 & 41 & 24.39 & 100.00 \\
\hline
\end{tabular}

HISTOGRAM FOR COLUMN 1 / FE PCT ।

2. OE $00 \times x$

3. OE $00 \quad \times \times \times \times \times \times \times \times \times \times \times \times \times \times \times \times \times$

5.0E $00 \quad x \times x \times x \times x \times x \times x \times x \times x \times x \times x \times x \times x \times x \times x \times$

7. OE $00 \quad x \times x \times x \times x \times x \times x \times x \times x \times x \times x \times$

er

1. UE $01 \times \times \times \times \times \times \times \times \times x$

1.5t $01 \quad x \times x \times x \times x \times x \times x \times x \times x \times x \times x \times x \times x \times$

$\begin{array}{ccccccc}N & L & H & G & T & 6 & \begin{array}{c}\text { ANALYTICAL } \\ \text { VALUES } \\ 0\end{array} \\ 0.0 & 0 & 0 & 0 & 0 & 0 & 41\end{array}$

MAXIMUM $=1.50000 E$ OI

MINIMUM $=2.00000 E$ OO

GEOMETRIC MEAN $=6.69323 \mathrm{E} 00$

GEOMETHIC DEVIATION $=1.80978 E 00$ 
FREOUEVCY TABLE FOR COLIJNN

$2+M G P C T$,

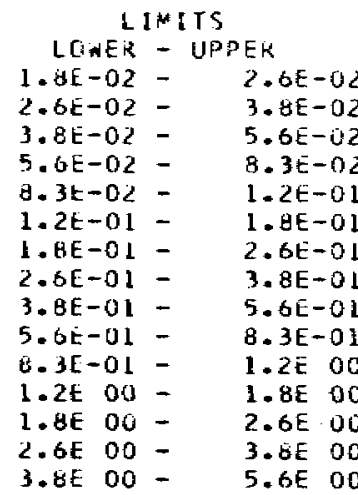

$\begin{array}{cc}\text { FREQ } & \text { FR } \\ & \text { C } \\ 0 & \\ 0 & \\ 0 & \\ 0 & \\ 0 & \\ 0 & \\ 0 & \\ 1 & \\ 0 & \\ 2 & \\ 4 & \\ 12 & 1 \\ 0 & 27 \\ 12 & 39 \\ 2 & 41\end{array}$

$\begin{array}{cc}\text { PERCENT } & \text { PERCENT } \\ \text { FREQ } & \text { FREQ CUM } \\ 0.0 & 0.0 \\ 0.0 & 0.0 \\ 0.0 & 0.0 \\ 0.0 & 0.0 \\ 0.0 & 0.0 \\ 0.0 & 0.0 \\ 0.0 & 0.0 \\ 2.44 & 2.44 \\ 0.0 & 2.44 \\ 4.80 & 7.32 \\ 4.76 & 17.07 \\ 29.27 & 46.34 \\ 19.51 & 65.85 \\ 29.27 & 95.12 \\ 4.88 & 100.00\end{array}$

HISTOGRAM FGR COJLUMN

2 \& MG PCT

\begin{abstract}
3.0E-01 $\times x$
\end{abstract}
$5.0 E-01$

7. OE-OL $\times \times \times \times \times$

$1.0 E \quad 00 \quad x \times x \times x \times x \times x x$

Uु

\title{
1. 5E $00 \quad x \times x \times x \times x \times x \times x \times x \times x \times x \times x \times x \times x \times x \times x \times x \times x$
}

2. Ot $00 \quad x \times x \times x \times x \times x \times x \times x \times x \times x \times x \times$

3.0E $00 \quad x \times x \times x \times x \times x \times x \times x \times x \times x \times x \times x \times x \times x \times x \times x \times x$

5.OE OO $\times \times \times \times \times$

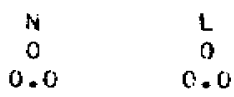

$\mathbf{H}$
0

B

$T$
0
0.0

ANAL YT ICAL

VALUES
41

MAXIMUM $=5.00000 E 00$

MINIHIJM $=3.00000 E-01$

GEQMETRIC MEAN $=1.83530 E$ OO

GEOMETRIC DEVIATIJN $=1.73197$ E 00 


\begin{tabular}{|c|c|c|}
\hline \multicolumn{3}{|c|}{ LEMITS } \\
\hline LOWEN & & PPER \\
\hline $3.8 E-02$ & - & $5.6 E-02$ \\
\hline $5.6 c-02$ & - & $9.3 \mathrm{E}-02$ \\
\hline A. JE -02 & - & $1.2 E-01$ \\
\hline $1.2 t-0.1$ & - & $1.8 \mathrm{E}-01$ \\
\hline 1. $8 \mathrm{E}-01$ & - & $2.6 \mathrm{E}-0 \mathrm{i}$ \\
\hline $2.6 \mathrm{E}-01$ & - & $3.8 \mathrm{E}-01$ \\
\hline 3. $.8 E-0 t$ & - & $5.6 E-01$ \\
\hline $5.6 \mathrm{E}-01$ & - & $8.3 E-01$ \\
\hline S. $3 E-01$ & - & $1.2 \mathrm{E} \mathrm{OO}$ \\
\hline $1.2 \mathrm{E} 0 \mathrm{O}$ & - & 1. $B E$ OO \\
\hline L.BE 00 & - & $2.6 E 00$ \\
\hline $2.6 \mathrm{E}$ OO & - & 3. $\mathrm{BE} 00$ \\
\hline
\end{tabular}

$\begin{array}{cc}\text { FREQ } & \text { FR } \\ & \text { C } \\ 0 & \\ 0 & \\ 0 & \\ 0 & \\ 0 & \\ 0 & \\ 0 & \\ 8 & \\ 3 & \\ 19 & 11 \\ 8 & 3 \\ 3 & 4 \\ & \end{array}$

$\begin{array}{ccc}\text { FREO } & \text { PERCENT } & \text { PERCENT } \\ \text { CUM } & \text { FREQ } & \text { FREQ CUM } \\ 0 & 0.0 & 0.0 \\ 0 & 0.0 & 0.0 \\ 0 & 0.0 & 0.0 \\ 0 & 0.0 & 0.0 \\ 0 & 0.0 & 0.0 \\ 0 & 0.0 & 0.0 \\ 0 & 0.0 & 0.0 \\ 8 & 19.51 & 19.51 \\ 11 & 7.32 & 26.83 \\ 30 & 46.34 & 73.17 \\ 38 & 19.51 & 92.68 \\ 41 & 7.32 & 10.000\end{array}$

HISTOGRAM FOR COLUMN

3 ( CA PCT ,

7. $0 \mathrm{E}-01 \times \times \times \times \times \times \times \times \times \times \times \times \times \times \times \times \times \times \times \times \times$

$1.0500 \quad x \times x \times x \times x$

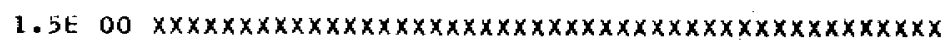

2.0E $00 \quad \times \times \times x \times x \times x \times x \times x \times \times \times \times \times \times \times x$

3. OE $00 \quad x \times \times \times \times \times x$

$\begin{array}{cc}N & L \\ 0 & 0 \\ 0.0 & 0.0\end{array}$

H

0.0

0.0

B

MAXIMUM $=3.00000 E 00$

MINIMUM $=7.0000 U E-01$

GEOHETRIC MEAN $=1.39646 \mathrm{E} 00$

GEOMETRIC DEVIATIUN = 1.52095 E 00 
FREUUENCY TABLE FOR CILUMN 4 ( TI PCT)

\begin{tabular}{|c|c|c|c|c|c|c|}
\hline \multicolumn{3}{|c|}{ LIMIIS } & FREQ & FREQ & PERCENF & PERCENT \\
\hline LOWER & - & ER & & CUM & FREQ & FREQ CUM \\
\hline $1.8 E-03$ & - & $2.6 E-03$ & 0 & 0 & 0.0 & 0.0 \\
\hline $2.6 E-0.5$ & - & $3.8 \mathrm{E}-03$ & 0 & 0 & 0.0 & 0.0 \\
\hline 3. $8 E-03$ & - & $5.6 E-03$ & 0 & 0 & 0.0 & 0.0 \\
\hline $5.6 \bar{E}-03$ & - & B. $3 E-03$ & 0 & 0 & 0.0 & 0.0 \\
\hline d. $3 E-03$ & - & $1.2 E-0.2$ & 0 & 0 & 0.0 & 0.0 \\
\hline $1.2 E-02$ & - & $1.8 t-02$ & 0 & 0 & 0.0 & 0.0 \\
\hline 1. $a E-02$ & - & $2.6 E-02$ & 0 & 0 & 0.0 & 0.0 \\
\hline $2.6 E-02$ & - & 3. $8 E-02$ & 0 & 0 & 0.0 & 0.0 \\
\hline $3.8 E-02$ & - & $5.5 E-02$ & 0 & 0 & 0.0 & 0.0 \\
\hline $5.6 E-02$ & - & $8.3 E-02$ & 0 & 0 & 0.0 & 0.0 \\
\hline $8 \cdot 3 t-02$ & - & $1.2 E-01$ & 0 & 0 & 0.0 & 0.0 \\
\hline $1.2 \mathrm{E}-01$ & - & 1. $8 E-01$ & 1 & 1 & 2.44 & 2.44 \\
\hline $1.8 E-01$ & - & $2.6 E-01$ & 3 & 4 & 7.32 & 9.76 \\
\hline $2.6 E-01$ & - & $3.8 E-01$ & 1.3 & 17 & 31.71 & 41.46 \\
\hline 3. $8 \mathrm{E}-01$ & - & $5.6 E-01$ & 9 & 26 & 21.95 & 63.41 \\
\hline $5.6 E-01$ & - & B. 3E-01 & 10 & 36 & 24.39 & 87.80 \\
\hline b. $3 E-01$ & - & $1.2 E 00$ & 5 & 41 & 12.20 & 100.00 \\
\hline
\end{tabular}

HISTOGKAM FOR COLUMIY

4 ( TI PCT)

$1.5 E-01 \quad x X$

2. OE-01 $\times \times \times \times \times \times \times$

3. $0 k-01 \quad x \times x \times x \times x \times x \times x \times x \times x \times x \times x \times x \times x \times x \times x \times x \times x \times$

5.0E-01 $\times x \times x \times x \times x \times x \times x \times x \times x \times x \times \times x \times x \times x$

厄]

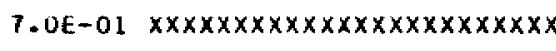

1. OE OO $\times x \times x \times x \times x \times x \times x$

$\begin{array}{ccccccc}N & L & H & 8 & T & 0 & \text { ANALYTICAL } \\ 0 & 0 & 0 & 0 & 0 & 0 & 41 \\ 0.0 & 0.0 & & & 0.0 & 0.0 & 4.0\end{array}$

MAXIMUH $=1.0000 \mathrm{UE} 00$

MINIMUM $=1.50000 E-01$

GEEMETRIC MEAN $=4.56152 \mathrm{E}-01$

GEOMETRIC DEVIATION $=1.67122 \mathrm{E} 00$ 
LIMITS
LULER - UPPER

LOWER - UPPER

Q.3E OO - 1.2E OI

1. HE OI - 2 - OE OI

2. $.6 E$ UI

2.6E OI- 3.6E O

3.8E O1 $-5.6 \mathrm{E} O$

H. 3E 01

8.3e O1 - 1.2E 02

1.2E $02-1.8 E E^{0}$

1.BE O2 - 2.6E O2

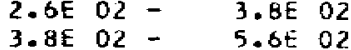

5.6E O2 - B. BE O

8.3E O2 - $1.2 E{ }^{0}$

1.2E $03=1.8 E O_{3}$

1.8E $03-2.6 E 03$

$\begin{array}{cc}\text { FRE } & \text { FR } \\ 0 & \\ 0 & \\ 0 & \\ 0 & \\ 0 & \\ 0 & \\ 0 & \\ 0 & \\ 0 & \\ 0 & \\ 0 & \\ 1 & \\ 5 & \\ 28 & \\ 5 & 34 \\ 2 & 41\end{array}$

FREQ

0.0

0.0

0.0

0.0

0.0
0.0

0.0

0.0

0.0

0.0
0.0

2.44

12.20

68.29

12.20

4.88
PERCENT

FREQ CUM

0.0

0.0

0.0

0.0

0.0

0.0

0.0

0.0

0.0

0.0

2.44

14.63

82.93

95.12

100.00

HISTOGRAM FOR COLUM:Y

51 MN PPM

7. UE $02 \times x$

1. OE $03 \quad \times \times \times \times \times \times \times \times \times \times \times x$

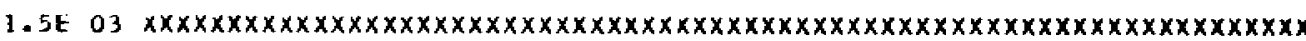

2.0E $03 \times x \times x \times x \times x \times x \times x$

g

$3.0 \mathrm{E} \quad 03 \times \times \times \times \times$

$\begin{array}{cc}14 & 1 \\ 0 & 0 \\ 0.0 & 0.0\end{array}$

H
0

MAXIMUM $=3.00000 E 03$

MIVIMUH $=7.00000 E 02$

GEOMETRIC MEAN $=1.50127 E 03$

GCOMETRIC DEVIATION $=1.30132$ E UO
ANALYT ICAL

VALUES

$\begin{array}{ccr}T & 6 & \text { VALUES } \\ 0 & 0 & 41 \\ 0.0 & 0.0 & \end{array}$


FREQUTENCY IABLE FUR COLUMN

6 I AG PPM I

$\begin{array}{ccccr}\text { LIMIIS } & \text { FREQ } & \text { FREQ } & \text { PERCENT } & \text { PERCENT } \\ \text { LUIER - UPPEK } & & \text { CUM } & \text { FREQ } & \text { FREQ CUM } \\ 3.8 L-01-5.6 E-01 & 2 & 2 & 4.88 & 4.88\end{array}$

HISTUGRAM FOR COLUMN

G I AG PPM

\section{UE-01 $\times \times \times \times x$}

$\begin{array}{ccccccc}N & \text { L } & \text { H } & B & T & 0 & \text { ANALYTICAL } \\ 30 & 9 & 0 & 0 & 0 & 0 & 2 \\ 73.17 & 21.95 & & & 0.0 & 0.0 & \end{array}$

MAXIMUH $=5.00000 E-01$

MINISHUM $=5.00000 E-01$

GEOMETKIC MEAN $=5.00000 E-01$

GEOMETRIC UEVIAFION $=1.00000$ 00 
FREDUENCY TABLE For COLUMN

7 AS PPM

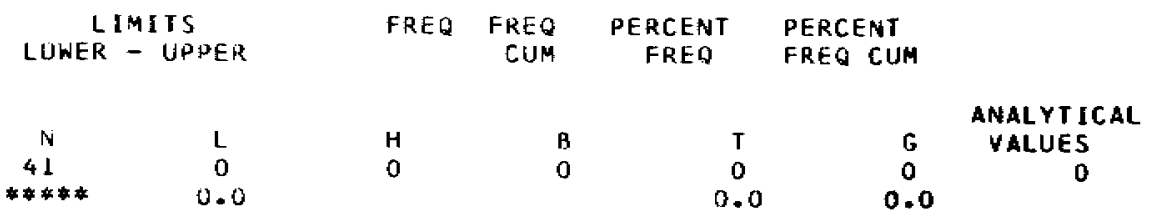

MAXIMUM $=-9.99900 E 48$

MIVIMUM $=9.99900 E 4 \mathrm{H}$

GEUMETRIC MEAN $=9.99900 E 48$

GEOMETRIC DEVIATION $=9.99900 E 48$ 


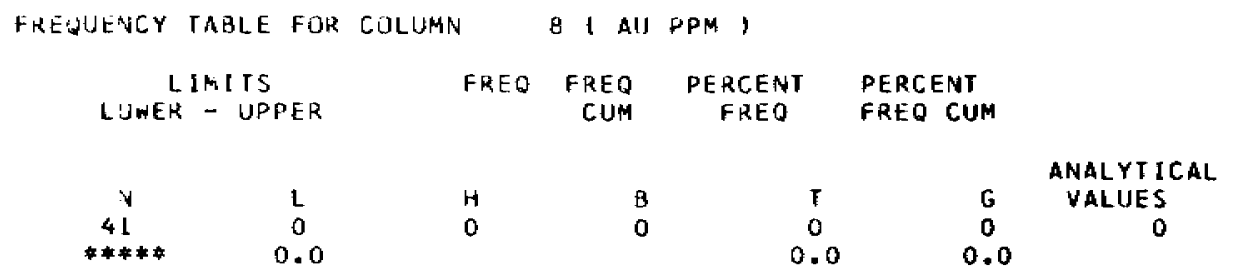

MAXIMUM $=-9.99400 E 48$

MINLIMUM $=9.99900 E 48$

GEOMETRIC MEAN $=9.99900 E 4 \mathrm{~B}$

GEOMETRIC DEVIATION $=9.99900 E 46$ 
FREQUENCY TABLE FOR COLUMN

9 ( B PPM)

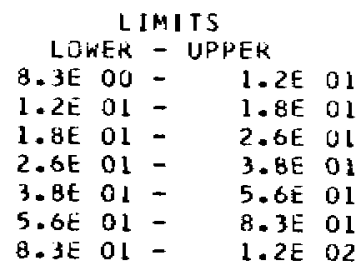

$\begin{array}{cc}\text { FRED } & \text { FRE } \\ 1 & \\ 6 & \\ 7 & \\ 13 & \\ 4 & \\ 3 & 3 \\ 2 & 36\end{array}$

$$
\begin{array}{rr}
\text { PERCENF } & \text { PERCENT } \\
\text { FREQ } & \text { FREQ CUM } \\
2.44 & 2.44 \\
14.63 & 17.07 \\
17.07 & 34.15 \\
31.71 & 65.85 \\
9.76 & 75.61 \\
7.32 & 82.93 \\
4.88 & 87.80
\end{array}
$$

HISTUgRAM fGR COLUMH 9 I B PPM

$1.0 E$ OL $x x$

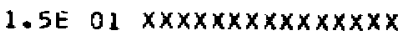

2. 0 E $01 \quad x \times \times \times \times \times \times \times \times \times \times \times \times \times \times \times x$

3.0E $01 \quad x \times x x \times x \times x \times x \times x \times x \times x \times x \times x \times x \times x x \times x \times x \times x \times x$

5. OE O1 $\times \times \times \times \times \times \times \times \times x$

7.OE $01 \quad x \times \times \times \times \times x$

1. UE $02 \quad x \times \times \times x$

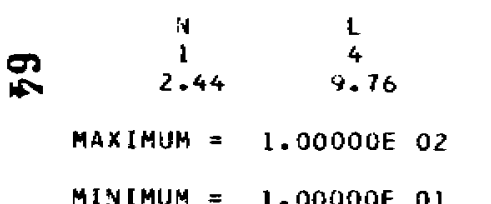

GEOMEIRIC MEAN $=2.90947 E$ OI

GEOMETRIC DEVIATION $=1.76532$ E OO 
FREQUEHCY TABLE FIIR COLUMN

10 ( ВА PРM)

\begin{tabular}{|c|c|c|c|c|}
\hline & & $M$ & & \\
\hline LD & $E \bar{E}$ & - & PEF & \\
\hline 1. $d E$ & 01 & & $2.6 E$ & 01 \\
\hline $2.6 \mathrm{E}$ & 01 & - & $3 . d \mathrm{E}$ & 01 \\
\hline $3.8 \mathrm{E}$ & 01 & - & $5.6 E$ & 01 \\
\hline $5.6 E$ & 01 & - & $B .3 E$ & 01 \\
\hline $5.3 \mathrm{E}$ & 01 & & $1.2 \mathrm{E}$ & 02 \\
\hline $1.2 E$ & 02 & & $1 . \hat{\mathrm{d} E}$ & 02 \\
\hline $1.8 \mathrm{E}$ & 02 & & $2.6 \mathrm{E}$ & 02 \\
\hline $2.6 E$ & 02 & & 3. $B E$ & 02 \\
\hline $3 . B E$ & 02 & & $5.6 \mathrm{E}$ & 02 \\
\hline $.6 E$ & 02 & & $8.3 E$ & 02 \\
\hline $8.3 E$ & 02 & - & $1.2 \mathrm{E}$ & 03 \\
\hline $1.2 \mathrm{E}$ & 03 & & 1. BE & 03 \\
\hline $1.8 \mathrm{E}$ & 03 & & $2.6 E$ & 03 \\
\hline $2.6 \mathrm{E}$ & 03 & & $3.9 E$ & 03 \\
\hline
\end{tabular}

$\begin{array}{cc}\text { FREQ } & F R E Q \\ & C \\ 0 & \\ 0 & \\ 1 & \\ 0 & \\ 0 & \\ 2 & \\ 7 & 1 \\ 10 & 2 \\ 3 & 2 \\ 6 & 29 \\ 4 & 3 \\ 5 & 38 \\ 1 & 39 \\ 2 & 41\end{array}$

$\begin{array}{cr}\text { PERCENT } & \text { PERCENT } \\ \text { FREQ } & \text { FREQ CUM } \\ 0.0 & 0.0 \\ 0.0 & 0.0 \\ 2.44 & 2.44 \\ 0.0 & 2.44 \\ 0.0 & 2.44 \\ 4.88 & 7.32 \\ 17.07 & 24.39 \\ 24.39 & 48.78 \\ 7.32 & 56.10 \\ 14.63 & 70.73 \\ 9.76 & 80.49 \\ 12.20 & 92.68 \\ 2.44 & 95.12 \\ 4.88 & 100.00\end{array}$

HISTOGRAM FOR COLUMN

10 I BA PPM

$5.0 E$ OI $x x$

7. OE 01

$1.0 E 02$

1.5E $02 \times \times \times \times \times$

2.0E $02 \quad \times \times \times \times \times \times x \times x \times \times \times \times \times \times \times x$

氶

3.0E $02 \quad x \times x \times x \times x \times x \times x \times x \times x \times x \times x \times x \times x x$

5. OE $02 \times x \times \times \times \times x$

7.0E $02 \quad x \times \times \times \times \times \times \times \times \times \times \times \times \times \times$

1. OE $03 \times \times \times \times \times \times \times \times \times \times$

1.5E $03 \times \times \times \times \times \times \times \times \times \times \times \times \times$

$2.0 \mathrm{E} 03 \times x$

$3.0 E \quad 03 \times \times \times \times \times$

$\begin{array}{ccccccc}N & L & H & B & T & G & \text { ANALYTICAL } \\ 0 & 0 & 0 & 0 & 0 & 0 & 41 \\ 0.0 & 0.0 & & & 0.0 & 0.0 & \end{array}$

MAXIMUH $=3.00000 E 03$

MINLMUM $=5.00000 E$ OL

GEOMETRIC MEAN $=4.88200 \mathrm{E} 02$

GEDMETRIC DEVIATION $=2.50213$ OO 
FREQUENCY TABLE FOR COLUMAN

11 BE PPM ]

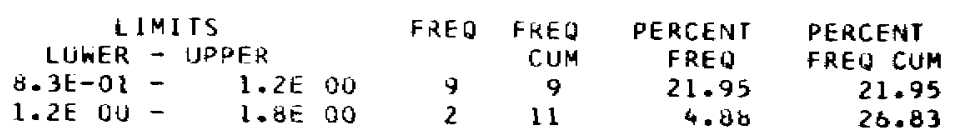

HISTOGRAM FOR COLUMN 1 I I BE PPM,

1. UE OO $x \times x \times x \times x \times x \times x \times x \times x \times x \times x \times x \times$

1. JE $00 \quad x \times \times \times x$

$\begin{array}{cc}N & 1 \\ 0 & 30 \\ 0.0 & 73.17\end{array}$

0

1
0
0.0

ANALYTICAL

$\begin{array}{cc}0.0 & 73.17 \\ \text { MUM }= & 1.50000 E \quad 00\end{array}$

MINIMUM $=1.00000$ E 00

GEOMETRIC MEAN $=1.07651$ E OO

GEOMETRIC DEVIATIUN = 1.17824 E OO 
FREGUEACY TABLE F!JR CULUMN

12 , BI PPM ।

\begin{tabular}{|c|c|c|c|c|c|c|}
\hline LIJWE & SPEA & FREO & $\begin{array}{r}\text { FREQ } \\
\text { CUMM }\end{array}$ & $\begin{array}{l}\text { PERCENT } \\
\text { FREQ }\end{array}$ & $\begin{array}{l}\text { PERCENT } \\
\text { FREQ CUM }\end{array}$ & \\
\hline$: d$ & $\mathrm{~L}$ & H & 8 & $\mathbf{T}$ & G & $\begin{array}{l}\text { ANALYTICAL } \\
\text { VALUES }\end{array}$ \\
\hline $\begin{array}{l}41 \\
* * * * *\end{array}$ & $\begin{array}{c}0 \\
0.0\end{array}$ & 0 & 0 & $\begin{array}{c}0 \\
0.0\end{array}$ & $\stackrel{0}{0.0}$ & 0 \\
\hline
\end{tabular}

MAXIMUM $=-9.99900$ E $4 B$

MINIMUM $=9.99900 E 4 \mathrm{~B}$

GEOMETRIC MEAL $=9.99900 E 48$

GEOMETRIC DEVIATION $=9.99900$ E $4 \mathrm{~B}$ 


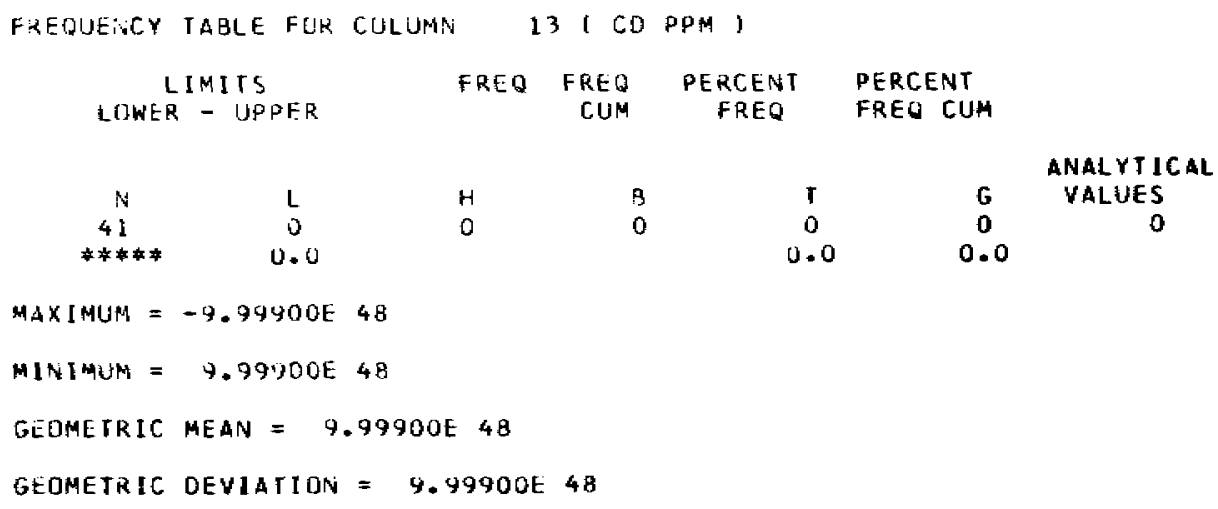

ฌ 
FREQUENCY FARLE FOR COLUMN

14 ( CO PPM

\begin{tabular}{|c|c|c|c|c|c|c|c|c|}
\hline \multicolumn{5}{|c|}{ LIMIIS } & FREQ & FREQ & PERCENT & PERCENT \\
\hline $1.8 \mathrm{E}$ & ou & - & $5.6 E$ & 00 & 2 & $\begin{array}{c}\text { CoM } \\
2\end{array}$ & $\begin{array}{l}\text { FREQ } \\
4.84\end{array}$ & FREA CUM \\
\hline $5.6 E$ & 00 & - & d. $3 E$ & 00 & 2 & 4 & 4.86 & 9.76 \\
\hline S. $3 E^{*}$ & 00 & - & $1.2 E$ & 01 & 3 & 7 & 7.32 & 17.07 \\
\hline $.2 \mathrm{E}$ & 01 & - & $1.8 E$ & 01 & 11 & 18 & 26.83 & \\
\hline $.6 E$ & 01 & - & $2.6 E$ & 01 & 7 & 25 & 17.07 & 60.98 \\
\hline $2.6 t$ & 01 & - & $3 . \mathrm{AE}$ & 01 & 11 & 36 & 26.83 & 87.80 \\
\hline $3.8 E$ & 01 & - & $5.6 \mathrm{E}$ & 01 & 4 & 40 & 9.76 & 97.56 \\
\hline $5.6 t$ & 01 & - & $8.3 E$ & 01 & 1 & 41 & 2.44 & 100.00 \\
\hline
\end{tabular}

HISTOGRAM FOR COLUMY

$14:$ CD PPM

5.0E $00 \times \times \times \times \times$

7. UE $00 \quad \times \times \times \times \times$

1. OE O1 $\times \times \times \times \times \times x$

1. 5t $01 \quad \times \times \times \times \times \times \times \times \times \times \times \times \times \times \times \times \times \times \times \times \times \times \times \times \times \times x$

2.0E $01 \quad x \times x \times x \times x \times x \times x \times x \times x \times x$

3. OE $01 \quad x \times x \times x \times x \times x \times x \times x \times x \times x \times x \times x \times x \times x \times x$

$5.0 E$ OL $x \times \times \times \times \times \times x \times x$

7. OE OL $\times X$

$B$

$\begin{array}{cc}N & L \\ 0 & 0 \\ 0.0 & 0.0\end{array}$

B
0

$T$
0
0.0

ANALYTICAL

VALUES

MAXIMUM $=7.00000 \mathrm{E} 01$

MINIMUM $=5.00000 E 00$

GEUMETRIC MEAN $=1.9642$ \#E 01

GEOMETRIC UEVIATIUN $=1.84947 E$ DO 
FREQUENCY TABLE FUR COLUMA $151 \mathrm{CR}$ PPM ,

\begin{tabular}{|c|c|c|c|}
\hline & & MI & \\
\hline $\mathrm{LO}$ & AE: & & DER \\
\hline $3.8 t$ & 00 & - & $5.6 \mathrm{E}$ \\
\hline $5.6 E$ & 00 & - & $9.3 E$ \\
\hline $3.3 E$ & 00 & - & $1.2 \mathrm{E}$ \\
\hline $1.2 \mathrm{E}$ & 01 & - & $1.8 \mathrm{E}$ \\
\hline $1.8 E$ & 01 & - & $2.6 \mathrm{E}$ \\
\hline $2.6 \mathrm{E}$ & nl & - & \\
\hline $.8 \mathrm{E}$ & 01 & - & $5.6 \mathrm{E}$ \\
\hline $.6 \mathrm{E}$ & 01 & - & \\
\hline $.3 E$ & 01 & - & $1.2 \mathrm{E}$ \\
\hline $1.2 t$ & 02 & - & $1.8 E$ \\
\hline & 02 & & \\
\hline
\end{tabular}

$\begin{array}{rr}\text { FRE } & \text { FR } \\ & \text { C } \\ 1 & \\ 0 & \\ 1 & \\ 1 & \\ 0 & \\ 5 & \\ 4 & 1 \\ 10 & 2 \\ 3 & 2 \\ 13 & 3 \\ 1 & 3\end{array}$

PERCENT

PERCENT

FREQ FREQ CUM

$\begin{array}{ll}2.44 & 2.44 \\ 0.0 & 2.44\end{array}$

$2.44 \quad 4.88$

$2.44 \quad 7.32$

$0.0 \quad 7.32$

$12.20 \quad 19.51$

$9.76 \quad 29.27$

$24.39 \quad 53.66$

$7.32 \quad 60.98$

$31.71 \quad 92.68$

$2.44 \quad 95.12$

HISTOGRAM FUR COLUMN 15 I CR PPM I

5. OE $0.0 \times x$

7. OE 00

$1.0 E 01 \times x$

$1.5 E 01 \times K$

2. OE 01

3.0E $01 \quad \times \times \times x \times x \times x \times \times x \times$

5. UE O1 $\times \times \times \times \times \times \times \times \times x$

$\exists \quad 7.0 \mathrm{E}$ ol $x \times x \times x \times x \times x \times x \times x \times x \times x \times x \times x \times x \times x \times x$

$1.0 \mathrm{E} \quad 02 \quad x \times x \times x \times x$

1.5E $02 \times x \times x \times x \times x \times x \times x \times x \times x \times x \times x \times x \times x \times x \times x \times x \times x \times x$

$2.0 E \quad 02 \times x$

$\begin{array}{ccccccc}N & \mathbf{L} & H & \mathrm{H} & T & G & \begin{array}{c}\text { ANALYTICAL } \\ \text { VALUES }\end{array} \\ 0 & 2 & 0 & 0 & 0 & 0 & 39 \\ 0.0 & 4.88 & & & 0.0 & 0.0 & \end{array}$

MAXIMUA $=2.00000 E 0.2$

MINIMUM $=5.00000$ E 00

GEOMETRIC MEAN $=7.05755 \mathrm{E}$ O1

GEOMETRIC DEVIATION $=2.29416$ E 00 
FREDUENCY TABLE FOR COLUMN 16 I CU PPM,

\begin{tabular}{|c|c|c|c|c|}
\hline & & $\mathrm{k}$ & & \\
\hline Lo & AEK & - & PER & \\
\hline $3.8 \mathrm{E}$ & 00 & - & $5.6 \mathrm{E}$ & 00 \\
\hline $5.6 \mathrm{E}$ & OU & - & $8.3 \mathrm{E}$ & 00 \\
\hline $8.3 \mathrm{E}$ & 00 & - & $1.2 \mathrm{E}$ & 01 \\
\hline $1.2 \mathrm{E}$ & 01 & - & $1.8 \mathrm{E}$ & ol \\
\hline $1.8 \mathrm{E}$ & 01 & - & $2.6 \mathrm{E}$ & 01 \\
\hline $2.6 \mathrm{E}$ & 01 & - & $3.8 E$ & 01 \\
\hline $3.8 E$ & 01 & - & $5.6 \mathrm{E}$ & 01 \\
\hline $5.6 \mathrm{E}$ & 01 & & $B .3 E$ & 01 \\
\hline $8.3 E$ & 01 & - & $1.2 E$ & 02 \\
\hline $1.2 \mathrm{E}$ & 02 & & $1.8 E$ & 02 \\
\hline 1. $\mathrm{BE}$ & 02 & - & $2.6 \mathrm{E}$ & 02 \\
\hline $2.6 \mathrm{E}$ & 02 & & $3.8 \mathrm{E}$ & 02 \\
\hline $3.8 E$ & 02 & - & $5.6 \mathrm{E}$ & 02 \\
\hline $.6 \mathrm{E}$ & 02 & - & 8. $3 \mathrm{E}$ & 02 \\
\hline
\end{tabular}

$\begin{array}{rr}\text { FREQ } & F R E O \\ 1 & \\ 0 & \\ 0 & \\ 4 & \\ 1 & \\ 6 & 1 \\ 7 & 19 \\ 11 & 30 \\ 3 & 3 \\ 4 & 3 \\ 1 & 38 \\ 1 & 39 \\ 0 & 39 \\ 1 & 40\end{array}$

$\begin{array}{cr}\text { PERCENT } & \text { PERCENT } \\ \text { FREQ } & \text { FREO CUM } \\ 2.44 & 2.44 \\ 0.0 & 2.44 \\ 0.0 & 2.44 \\ 9.76 & 12.20 \\ 2.44 & 14.63 \\ 14.63 & 29.27 \\ 17.07 & 46.34 \\ 26.83 & 73.17 \\ 7.32 & 80.49 \\ 9.76 & 90.24 \\ 2.44 & 92.68 \\ 2.44 & 95.12 \\ 0.0 & 95.12 \\ 2.44 & 97.56\end{array}$

HISTOGRAM FOR CULUMN

16 ( CU PPM

5. 0 E $00 \times x$

7.0E 00

1. OE 01

1.5E $01 \quad \times \times \times \times \times \times \times \times \times \times$

$2.0601 \times x$

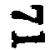

3. OE $01 \times \times \times \times \times \times \times \times \times \times \times \times \times \times \times$

5.0E $01 \quad x \times x \times x \times x \times x \times x \times x \times x \times x$

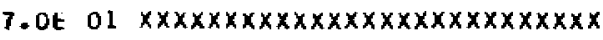

1. UE $02 \times \times \times \times \times \times x$

1. SE $02 \times \times \times \times \times \times \times \times \times x$

$2.0 E 02 \times x$

3.0E $02 \times x$

$5.0 \mathrm{E} \mathrm{O2}$

7. OE $02 \times X$

\begin{tabular}{ccccccc}
$N$ & $L$ & $H$ & & & \multicolumn{2}{c}{ ANALYTICAE } \\
0 & 1 & 0 & 0 & $T$ & $G$ & VALUES \\
0.0 & 2.44 & & & 0 & 0 & 40
\end{tabular}

MAXIMUM $=7.00000 E 02$

MINIMUM $=5.00000 E 00$

GEOMETHIC MEAIA $=5.65045 E$ OI 
GEOMETRIC DEVIATION $=2.49219 E$ OO

\section{$\vec{N}$}


FREQUENCY TABLE FOR COLUMA

17 I LA PPM )

\begin{tabular}{|c|c|c|c|c|c|c|c|c|}
\hline LO & \multicolumn{4}{|c|}{ LIMITS } & FREQ & $\begin{array}{r}\text { FREQ } \\
\text { CUM }\end{array}$ & $\begin{array}{c}\text { PERCENT } \\
\text { FREO }\end{array}$ & $\begin{array}{l}\text { PERCENT } \\
\text { FREQ CUM }\end{array}$ \\
\hline $1.8 \mathrm{E}$ & 01 & - & $2.6 E$ & 01 & 1 & l & 2.44 & 2.44 \\
\hline $2.6 \mathrm{E}$ & ol & - & $3.8 E$ & 01 & 2 & 3 & 4.88 & 7.32 \\
\hline $1.8 \mathrm{E}$ & 01 & - & $5.6 \mathrm{E}$ & 01 & 0 & 3 & 0.0 & 7.32 \\
\hline $5.6 E$ & 01 & - & $8.3 E$ & 01 & 0 & 3 & 0.0 & 7.32 \\
\hline $8.3 E$ & 01 & - & $1.2 \mathrm{E}$ & 02 & 0 & 3 & 0.0 & 7.32 \\
\hline $1.2 \mathrm{E}$ & 02 & - & $1.8 \mathrm{E}$ & 02 & 1 & 4 & 2.44 & 9.76 \\
\hline
\end{tabular}

HISTOGRAM FOR COLUMN 17 ( LA PPM , 2.0E $01 \times x$

3. OE $01 \times \times \times x \times$

5. OE Ol

7.OE O1

1. OE 02

1. SE $02 \times x$

$\begin{array}{ccccccc}N & L & H & & & & \text { ANALYTICAL } \\ 3 & 34 & 0 & 0 & T & G & \text { VALUES } \\ 7.32 & 82.93 & & 0 & 0 & 0 & 4\end{array}$

MAXIMUM $=1.50000 E 02$

W MINIMUM $=2.00000$ E 01

GEOMETRIC MEAN $=4.05354 E 01$

GEOMETRIC DEVIATION $=2.44242 \mathrm{E}$ 00 
Frequency table FGR COLUMN

18 МO РРM ।

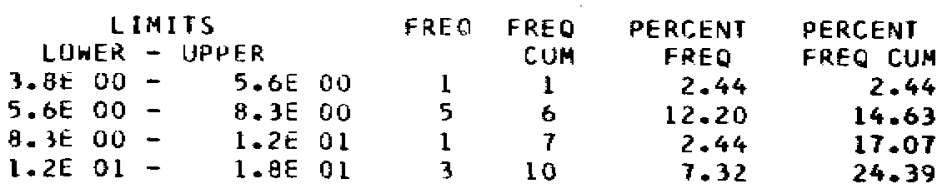

HISTOGRAM FOR COLUMN 18 \{ MOPPM,

5.0E $00 \times x$

T. $0 \mathrm{E} \quad 00 \quad \times \times \times \times \times \times \times \times \times \times \times \times$

1. OE $01 \times x$

1.5E $01 \times \times \times \times \times \times x$

$\begin{array}{cc}N & L \\ 8 & 23 \\ 19.51 & 56.10\end{array}$

19.5

56.10

MAXIMUM $=1.50000 E 01$

MINIMUH $=5.00000 E$ OO

GLOMEIRIC MEA.N $=8.81602 E 00$

$\vec{N}$

GEDMETRIC DEVIATION $=1.49409 E 00$ 
FRESUEVCY TABLE FER COLUMN

$$
\begin{array}{cccccc}
\text { LIMITS } & \text { FREQ } & \text { FREQ } & \text { PERCENT } & \text { PERCENT } \\
\text { LUGER - UPPER } & & & \text { CUM } & \text { FREQ } & \text { FREQ CUM } \\
8.3 E 00- & 1.2 E 01 & 29 & 29 & 70.73 & 70.73 \\
1.2601- & 1.8 E 01 & 5 & 34 & 12.20 & 82.93
\end{array}
$$

HISTOGRAM FOR COLUMN LI A NB PPM )

1.0E OI $\times \times \times \times \times \times \times \times \times \times \times \times \times \times \times \times \times \times \times \times \times \times \times \times \times \times \times \times \times \times \times \times \times \times \times \times \times \times \times \times \times \times \times \times \times \times \times \times \times \times \times \times \times \times \times \times \times \times \times \times \times \times \times \times \times \times \times \times \times \times \times$

1. $5 \mathrm{E}$ OI $\times \times \times \times \times \times \times \times \times \times \times$

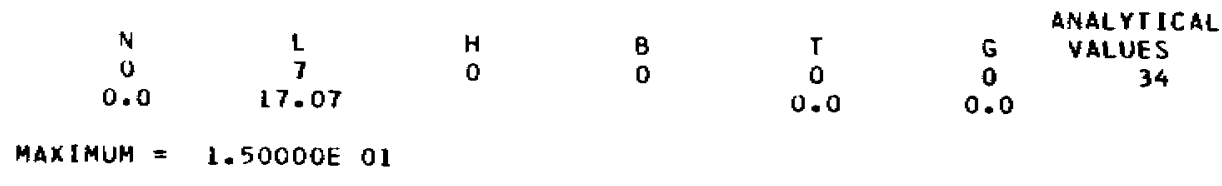

MINIMUM $=1.00000 E 01$

GEOMETRIC MEAN $=1.06142 \mathrm{E} 01$

GEOMETRIC DEVIATION = $1.15709 E$ OO

जै 


\begin{tabular}{|c|c|c|c|c|c|c|c|}
\hline \multicolumn{4}{|c|}{ LIMITS } & FREQ & $\begin{array}{l}\text { FREQ } \\
\text { CUNA }\end{array}$ & $\begin{array}{c}\text { PERCENI } \\
\text { FREQ }\end{array}$ & $\begin{array}{l}\text { PERCENT } \\
\text { FREO CUM }\end{array}$ \\
\hline $3.6 \mathrm{E} \quad 0$ & 00 & $5.6 \mathrm{E}$ & 00 & 1 & 1 & 2.44 & 2.44 \\
\hline $5.6 E$ & vo - & $8.3 E$ & 0.0 & 2 & 3 & 4.88 & 7.32 \\
\hline $.3 \mathrm{E}$ & $00-$ & $1.2 \mathrm{E}$ & 01 & 1 & 4 & 2.44 & 9.76 \\
\hline $.2 E$ & 01 - & $1.8 \mathrm{E}$ & 01 & 2 & 6 & 4.80 & 14.63 \\
\hline BE 0 & 01 & $2.6 \mathrm{E}$ & 01 & 1 & 7 & 2.44 & \\
\hline GE 0 & 01 & 3. at & 01 & 18 & 25 & 43.90 & 60.98 \\
\hline 3. $\mathrm{dE}$ & 01 & $5.6 E$ & 01 & 3 & $2 \theta$ & 7.32 & 68.29 \\
\hline $5.6 \mathrm{E}$ & $01-$ & $8.3 E$ & 01 & 13 & 41 & 31.71 & 100.00 \\
\hline
\end{tabular}

HISTOGRAM FUR CULUMN

201 NI PPM

$5.0 E 00 \times x$

7.0E $00 \times \times \times \times x$

1. OE OI $X x$

$1.5 E$ OI $\times \times \times \times x$

2.0E $01 x x$

3.0E $01 \quad x \times x \times x \times x \times x \times x \times x \times x \times x \times x \times x \times x \times x \times x \times x \times x \times x \times x \times x \times x \times x \times x \times$

5.0E $01 \times \times \times \times \times \times \times$

7. OE O1 $x \times x \times x \times x \times x \times x \times x \times x \times x \times x \times x \times x \times x \times x \times x \times x \times x x$

$\begin{array}{cc}N & L \\ O & 0 \\ 0.0 & 0.0\end{array}$

$H$
0

B

1
0
0.0

ANALYTICAL

VALUES

MAXIMUM $=7.00000 E$ OI

MINIMUH $=5.00000 E$ DO

GEOMETRIC MEAN $=3.38530 E 01$

GEOMETRIC DEVIATION $=2.00286 E 00$ 
FREQUENCY TABLE FOR CULUMN

$21 \mid P B P P M$ ।

\begin{tabular}{|c|c|c|c|c|c|c|c|c|}
\hline \multicolumn{5}{|c|}{ LIMIIS } & \multirow[t]{2}{*}{ FKED } & \multirow{2}{*}{$\begin{array}{l}\text { FREQ } \\
\text { CUM }\end{array}$} & \multirow{2}{*}{$\begin{array}{c}\text { PERCEN } \\
\text { FREQ }\end{array}$} & \multirow{2}{*}{$\begin{array}{l}\text { PERCENT } \\
\text { FREO CUM }\end{array}$} \\
\hline $8.3 E$ & $\begin{array}{l}\text { AER } \\
\text { OO }\end{array}$ & - & EER & 01 & & & & \\
\hline $1.2 \mathrm{E}$ & 01 & - & PF & 01 & 14 & 19 & 12.20 & 12.20 \\
\hline $1.8 \mathrm{E}$ & 01 & - & $2.6 E$ & 01 & $\mathrm{e}$ & 21 & 19.51 & $\begin{array}{l}46.34 \\
65.85\end{array}$ \\
\hline $.6 \mathrm{E}$ & 01 & - & $3.8 \mathrm{E}$ & 01 & 5 & 32 & 12.20 & $\begin{array}{l}03000 \\
78.05\end{array}$ \\
\hline $3.8 \mathrm{E}$ & 01 & - & $5.6 \mathrm{E}$ & 01 & 1 & 33 & 2.44 & 80.49 \\
\hline & OI & - & $8.3 \mathrm{E}$ & 01 & 3 & 36 & 7.32 & 87.80 \\
\hline $3 E$ & OI & - & $1.2 \mathrm{E}$ & 02 & 1 & 37 & 2.44 & \\
\hline $2 E$ & 02 & - & $1.8 E$ & 02 & 2 & 39 & $4.8 B$ & 95.12 \\
\hline
\end{tabular}

histugram fur culuma 21 I PB ppm ,

1. OE OI $\times \times \times \times \times \times \times \times \times \times \times x$

1.5E 01 $x \times x \times x \times x \times x \times x \times x \times x \times x \times x \times x \times x \times x \times x \times x \times x \times x \times$

2.OE $01 \quad x \times x \times x \times x \times x \times x \times x \times x \times x \times x x$

3.OE $01 \quad x \times x \times x \times x \times x \times x \times$

5. OE $01 \times x$

7. OE $01 \times \times \times \times \times \times x$

$1.0 E \quad 02 \times x$

1.5E $02 \times \times \times \times \times$

ปี

$\begin{array}{cc}N & L \\ 0 & 2 \\ 0.0 & 4.68\end{array}$

$H$
0

MAXIMUM $=1.50000 E$ O2

MINIMUM = $1.00000 E$ OI

GEDMETRIC MEAN $=2.26469 \mathrm{E} \mathrm{Ol}$

GEOMETRIC DEVIATION $=2.06719 E$ DO
ANALYTICAL

VALUES
39 


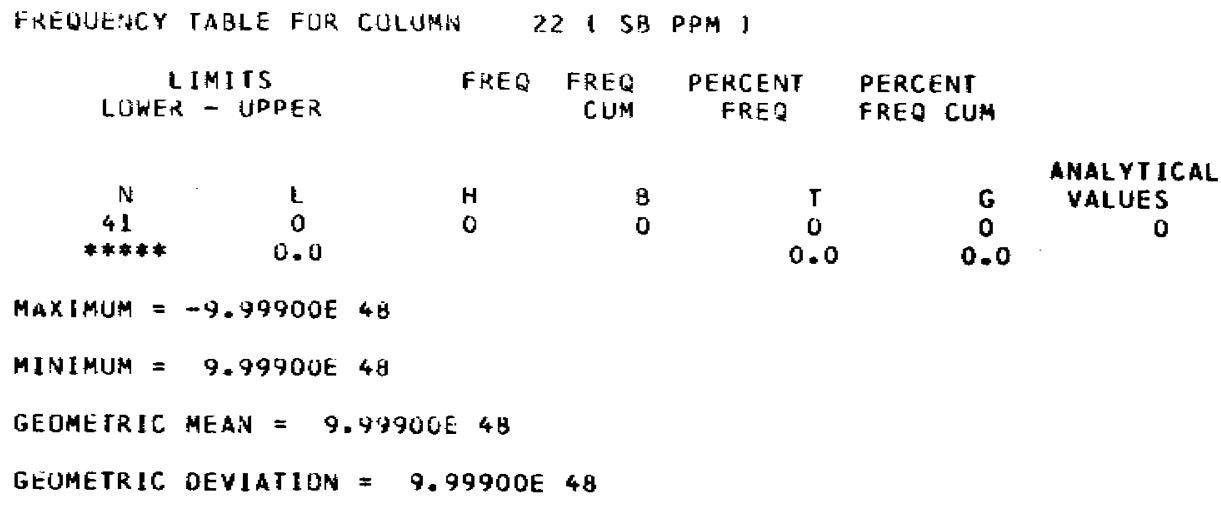

ప 
FREQUENCY TABLE FOR COLUMN 23 I SC PPM )

\begin{tabular}{|c|c|c|c|c|c|c|c|c|}
\hline \multicolumn{5}{|c|}{ LIMITS } & FREQ & $\begin{array}{l}\text { FREQ } \\
\text { CUM }\end{array}$ & $\begin{array}{c}\text { PERCENT } \\
\text { FREO }\end{array}$ & $\begin{array}{l}\text { PERCENT } \\
\text { FREO CU: }\end{array}$ \\
\hline 3.81 & 00 & - & $5.6 \mathrm{E}$ & 00 & 1 & 1 & 2.44 & 2.44 \\
\hline $5.6 \mathrm{E}$ & 00 & - & $8.3 \mathrm{E}$ & 00 & 2 & 3 & 4.88 & 7.32 \\
\hline g. 3t & 00 & - & $1.2 \mathrm{E}$ & 01 & 3 & 6 & 7.32 & 14.63 \\
\hline $1.2 E$ & 01 & - & $1.8 E$ & 01 & 14 & 20 & 34.15 & 48.78 \\
\hline $1.0 E$ & 01 & - & $2.6 E$ & 01 & 5 & 25 & 12.20 & 60.98 \\
\hline $.6 \mathrm{E}$ & 01 & - & $3.8 \mathrm{E}$ & 02 & 14 & 39 & 34.15 & 95.12 \\
\hline
\end{tabular}

HISTDGRAM FOR COLUMN 231 SC PPM

5. OE DOXX

7. OE OO $\times \times \times \times \times$

$1.0 E 01 \times \times \times \times \times \times x$

1.5E $01 \quad x \times x \times x \times x \times x \times x \times x \times x \times x \times x \times x \times x \times x \times x \times x \times x \times x \times x \times$

2. $0 E \quad 01 \times \times \times \times \times \times \times \times \times \times \times \times \times$

3.0E 01 $x \times x \times x \times x \times x \times x \times x \times x \times x \times x \times x \times x \times x \times x \times x \times x \times x \times$

$\begin{array}{ccccccc}N & L & H & 0 & T & G & \text { ANALYTICAL } \\ 0 & 2 & 0 & 0 & 0 & 0 & \text { VALUES } \\ 0.0 & 4.88 & & & 0.0 & 0.0 & 39\end{array}$

MAXIMUM $=3.00000 E$ OI

Co minimum $=5.00000$ E 00

GEOMETRIC MEAN $=1.80889 E$ OL

GEOMETRIC OEVIATION = $1.61080 E$ OO 
Frequency table fur culuma

24 ( SN PPM)

\begin{tabular}{|c|c|c|c|c|c|c|c|c|}
\hline \multicolumn{5}{|c|}{ LIMIIS } & \multirow[t]{2}{*}{ FYEQ } & \multirow{2}{*}{$\begin{array}{l}\text { FREO } \\
\text { CUM }\end{array}$} & \multirow{2}{*}{$\begin{array}{c}\text { PERCENT } \\
\text { FREQ }\end{array}$} & \multirow{2}{*}{$\begin{array}{l}\text { PERCENT } \\
\text { FREQ CUMM }\end{array}$} \\
\hline & NER & - & PER & & & & & \\
\hline $8.3 E$ & 00 & - & $1.2 \mathrm{E}$ & ol & 2 & 2 & 4.88 & 4.88 \\
\hline $1.2 \mathrm{E}$ & 01 & - & 1. $8 \mathrm{E}$ & 01 & 3 & 5 & 7.32 & 12.20 \\
\hline $1.6 \mathrm{E}$ & 01 & - & $2.6 \mathrm{E}$ & 01 & 1 & 6 & 2.44 & 14.63 \\
\hline $2.6 \mathrm{E}$ & 02 & - & 3. $8 E$ & 01 & 3 & 9 & 7.32 & 21.95 \\
\hline $3.8 \mathrm{E}$ & 01 & - & $5.6 \mathrm{E}$ & ol & 0 & 9 & 0.0 & 21.95 \\
\hline $5.6 E$ & 01 & - & B. $3 E$ & 01 & 1 & 10 & 2.44 & 24.39 \\
\hline $3 E$ & 01 & - & $1.2 \mathrm{E}$ & 02 & 1 & 11 & 2.44 & 26.03 \\
\hline
\end{tabular}

HISTUGRAM FOR COLUMN 24 \{ SN PPM ,

1. OE OI $x \times \times \times x$

1.5E $01 \quad x \times \times x \times x \times$

$2.0 E 01 \times x$

3. OE O1 $x \times x \times x \times x$

$5.0 E$ OI

T. OE OI $\times X$

1. DE $02 \times x$

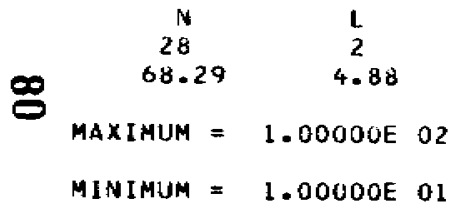

GEOMETRIC MEAY = $2.36183 E$ OI

GLOMETRIC DEVIATION $=2.10749 E 00$ 
F:SEQUENCY TABLE FIR COLUMA 25 I SR PPM I

\begin{tabular}{|c|c|c|c|c|c|c|c|}
\hline \multicolumn{4}{|c|}{ LIMITS } & FRE? & FREQ & PERCENT & $\begin{array}{l}\text { PERCENT } \\
\text { FREU CUM }\end{array}$ \\
\hline $8.3 E 0$ & $01-$ & $1.2 \mathrm{E}$ & 02 & 0 & $\operatorname{lom}_{0}$ & $\begin{array}{r}\text { FREO } \\
0.0\end{array}$ & 0.0 \\
\hline $1.2 t 0$ & $02-$ & $1.8 \mathrm{E}$ & u2 & 0 & 0 & 0.0 & 0.0 \\
\hline $1.8 \mathrm{E} \quad 0$ & $02-$ & $2.6 \mathrm{E}$ & 02 & 4 & 4 & 9.76 & 9.76 \\
\hline $2.6 E \quad 0$ & $02-$ & $3.8 \mathrm{E}$ & 02 & 29 & 32 & 68.29 & 78.05 \\
\hline 1. $8 \mathrm{E} \quad 0$ & $02-$ & $5.6 \mathrm{E}$ & 02 & A & 40 & 19.51 & 97.56 \\
\hline $6 \mathrm{E} \quad 0$ & $02-$ & $8.3 \mathrm{E}$ & 02 & 1 & 41 & 2.44 & 100.00 \\
\hline
\end{tabular}

HISTOGRAM FUR COLUMN 25 I SR PPM ,

$2.0 \mathrm{E} \quad 02 \times \times \times \times \times \times \times \times \times \times x$

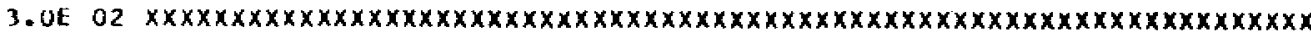

5.0E $02 \times x \times x \times x \times x \times x \times x \times x \times x \times \times \times x$

7. OE $02 \times x$

$\begin{array}{ccccccc}N & L & H & B & T & G & \text { ANALYTICAL } \\ 0 & 0 & 0 & 0 & 0 & 0 & 41 \\ 0.0 & 0.0 & & & 0.0 & 0.0 & \end{array}$

MAXIMUM $=7.00000 \mathrm{E} 02$

MINIMUM $=2.00000 E \quad 02$

GLOMETRIC MEAN $=3.25234 E$ O2

$\infty$

Geumetric ueviation = 1.32702 eo 
FREQUENCY TAGLE FUR [IJLUMY 25 I V PPM ]

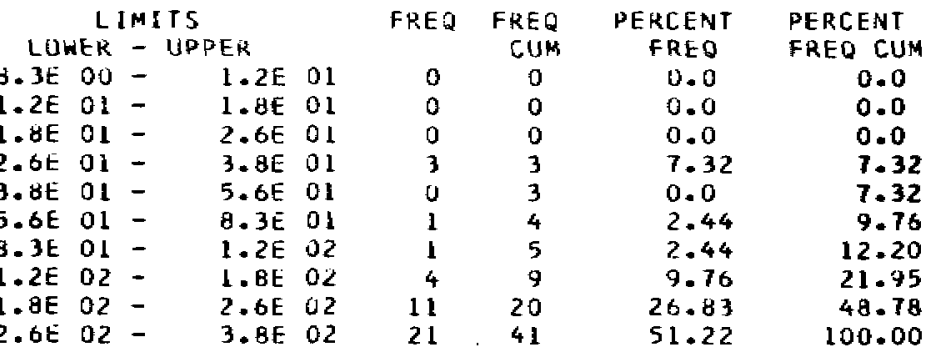

HISTOGRAM FOR CULUMN 26,1 V PPM I

3. OE $01 \times \times \times \times \times \times \times$

5. OE OL

7. OE $01 \times x$

$1.0 \mathrm{E} 02 \times \mathrm{X}$

1. 5E $02 \quad x \times x \times x \times x \times x \times$

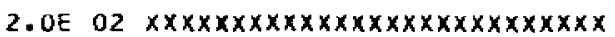

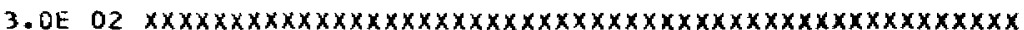

思

$\begin{array}{cc}N & L \\ 0 & 0 \\ 0.0 & 0.0\end{array}$

$\mathbf{H}$
$\mathbf{0}$

B

$T$
0
0.0

ANALYTICAL

VALUES

MAXIMUM $=3.00000 E 02$

MINIHUM $=3.00000$ E OI

GEOHETRIC MEAN $=1.49654$ E 02

GLOMETRIC DEVIATIUN $=1.88850 E$ DO 
FREQUENCY TATLE FUR CILUMN

27 ( W PPM )

LIMITS
LOWER - UPPER

FQED FREQ PERCENT

CUM FRER

PERCENT

$4 \mathrm{~L}$

$\mathbf{H}$
$\mathbf{0}$

3

$T$
0

FREQ CUM

**** 0.0

MAXIMUM $=-9.99900 E 48$

MINIMUM $=9.99900 E 49$

GEOMETRIC MEAN $=9.94400 E 48$

GEOMETRIC DEVIATIUN $=9.99900 E 46$ 
FrREUENCY TAHLE FUK COLUMN 29 , Y PPM ,

\begin{tabular}{|c|c|c|c|c|c|c|c|}
\hline \multicolumn{4}{|c|}{ LIMITS } & FPEO & FREQ & PERCENT & PERCENT \\
\hline LJWEr & - & DERK & & & CUM & FREQ & FREQ CUM \\
\hline 8. 3E 0 & $\begin{array}{l}- \\
-\end{array}$ & $1.2 \mathrm{E}$ & 01 & 5 & 5 & 12.20 & 12.20 \\
\hline $1.2 \mathrm{t}$ & - & $1 . B E$ & 01 & 17 & 22 & 41.46 & 53.66 \\
\hline $1.8 E$ & - & 2.65 & ol & 14 & 36 & 34.15 & 87.80 \\
\hline $2.6 \mathrm{E}$ & - & $3.6 \mathrm{E}$ & 01 & 5 & 41 & 12.20 & 100.00 \\
\hline
\end{tabular}

HISTOGRAM FOR CCLUMN

$201 \quad Y P P M$

1. Ot $01 \quad \times \times x \times x \times x \times x \times x \times$

1.5E $01 \quad x \times x \times x \times x \times x \times x \times x \times x \times x \times x \times x \times x \times x \times x \times x \times x \times x \times x \times x \times x \times x \times$

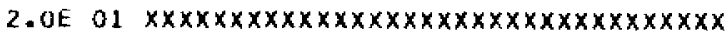

3.01 01 $\times x \times x \times x \times x \times x \times x$

$\begin{array}{ccccccc}\text { N } & \text { L } & H & & & & \text { ANALYTICAL } \\ 0 & 0 & 0 & 0 & T & G & \text { VALUES } \\ 0.0 & 0.0 & & 0 & 0 & 0 & 41\end{array}$

MAXIMUM $=3.00000 E 01$

MINIMUM $=1.00000 \mathrm{E}$ 01

GEOMETRIC MEAN $=1.71390 \mathrm{E}$ OI

GEOMETRIC DEVIATION $=1.35296 \mathrm{E}$ O0

$\infty$ 
FREQUEHCY TABLE FIJR COLUMA 29 ( ZMS PPM 1

$\begin{array}{ccccrr}\text { LIMIIS } & \text { FREQ FREQ } & \text { PERCENT } & \text { PERCENT } \\ \text { LOWER - UPPER } & & \text { CUM } & \text { FREQ } & \text { FREO CUM } \\ 1.0 E 02- & 2.6 E 02 & 5 & 5 & 12.20 & 12.20 \\ 2.6 E 02- & 3.8 E 02 & 2 & 7 & 4.88 & 17.07\end{array}$

HISTOGRAM FOR COLUMN 29 I ZN PPM ,

2.0E $02 \times x \times x \times x \times x \times x \times x$

3. OE $02 \times \times \times \times \times$

$\begin{array}{ccccccc}N & L & H & & & & \text { ANALYTICAL } \\ 1 & 33 & H & 0 & 0 & G & \text { VALUES } \\ 2.44 & 80.49 & 0 & 0 & 0.0 & 0.0 & 7\end{array}$

MAXIMUM $=3.00000 E 02$

MINIMUM $=2,00000 E 02$

GEOMETRIC MEAN $=2.24563$ E 02

GEOMETRIC DEVIATION $=1.21896 \mathrm{E} 00$ 


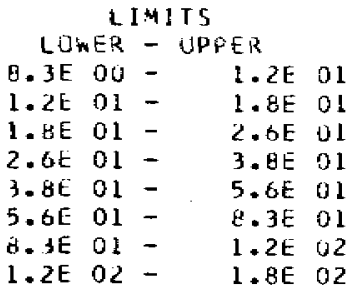

$\begin{aligned} & \text { FRt } \text { P } \\ & 0 \\ & 0 \\ & 0 \\ & 0 \\ & 2 \\ & 31 \\ & 7 \\ & 1\end{aligned}$

$\begin{array}{ccc} & \\ \text { PED } & \text { PERCENT } & \text { PERCENT } \\ \text { CIIM } & \text { FREQ } & \text { FREQ CUM } \\ 0 & 0.0 & 0.0 \\ 0 & 0.0 & 0.0 \\ 0 & 0.0 & 0.0 \\ 0 & 0.0 & 0.0 \\ 2 & 4.88 & 4.88 \\ 33 & 75.61 & 80.49 \\ 40 & 17.07 & 97.56 \\ 41 & 2.44 & 100.00\end{array}$

HISTOGRAM FOR COLUMN

$30(2 K$ PPM

5.0E $02 \times \times \times \times x$

7.0E $01 \quad x \times x \times x \times x \times x \times x \times x \times x \times x \times x \times x \times x \times x \times x \times x \times x \times x \times x \times x \times x \times x \times x \times x \times x \times x \times x \times x \times x \times x \times x \times x \times x \times x \times x \times x \times x \times x \times x \times x \times$

1.0E $02 \quad x \times x \times x \times x \times x \times x \times x \times x \times x$

1. bE $02 \times x$

$\begin{array}{ccccccc}N & L & H & B & T & G & \text { ANALYIICAL } \\ 0 & 0 & 0 & 0 & 0 & 0 & 41 \\ 0.0 & 0.0 & & & 0.0 & 0.0 & \end{array}$

MAXIMUM $=1.50000 E 02$

MINIMUM $=5.00000 E$ O

GLOMETRIC MEAN $=7.45563 \mathrm{E} 01$

GEDMETRIC DEVIATIUN $=1.21645$ E 00 
FRELUENCY TABLE FLR COLUMN

31 I AU PPM I

\begin{tabular}{|c|c|c|c|c|c|c|}
\hline \multicolumn{3}{|c|}{ LI 1 II 5} & FRED & FRE & PERCENT & PERCENT \\
\hline LOWEK & - & PER & & cum & FREO & FREQ CU:A \\
\hline $1.8 E-02$ & - & $2.6 \mathrm{E}-02$ & 0 & 0 & 0.0 & 0.0 \\
\hline $2.6 \mathrm{E}-02$ & - & 3. $8 E-02$ & 0 & 0 & 0.0 & 0.0 \\
\hline $3.8 E-02$ & - & $5.6 \mathrm{E}-02$ & 0 & 0 & 0.0 & 0.0 \\
\hline $5.6 \mathrm{E}-\mathrm{O} 2$ & - & $8.3 E-02$ & ) & 0 & 0.0 & 0.0 \\
\hline $9 \cdot 3 t-02$ & - & $1.2 \mathrm{E}-01$ & 1 & l & 2.44 & 2.44 \\
\hline $1-2 E-01$ & - & 1. $\Delta E-01$ & 0 & 1 & 0.0 & 2.44 \\
\hline $1.8 E-01$ & - & $2.6 t-01$ & 0 & 1 & 0.0 & 2.44 \\
\hline $2.6 t-01$ & - & $3.8 E-01$ & 1 & 2 & 2.44 & 4.88 \\
\hline
\end{tabular}

HISTOGRAM FDR COLUMN 31 , AU OPM ।

$$
1 \text {. Ot }-01 \times x
$$$$
\text { 1. SE-O1 }
$$

$2.0 E-01$

3. $0 E-01 \times x$

$\begin{array}{ccccccr}N & L & H & B & & & \text { ANALYTICAL } \\ 0 & 39 & 0 & 0 & 0 & 0 & 2 \\ 0.0 & 95.12 & & & 0.0 & 0.0 & \end{array}$

MAXIMUM $=3.00000 E-01$

MINIMUM = 1.00OOUE-0I

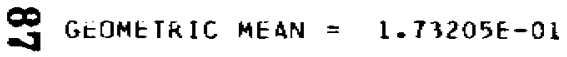

GEJMEJRIC DEVIATION $=2.17458$ OO 
ELEML: $T$

FE PCI

MU PCI

CA PCT

I I PCI

MN PPM

AS PPM

AS PPM

AL $P P M$

Q $P P M$

DA PPM

DI PPM

CD PPM

CO $P P M$

CR PPM

CU PPM

LA PPM

MO PPM

IS PPM

NI $P P$ i

PB PPM

$\$ B \quad P P M$

$S C P P M$

$S N P P M$

$S R P P M$

$P P P M$

WPPM

C. $\angle N P P M$

ZR PPM

AU PPM

\begin{tabular}{|c|c|c|c|}
\hline \multirow{2}{*}{\multicolumn{2}{|c|}{ ELEMEN T }} & \multirow{2}{*}{$\begin{array}{l}\text { GEOME TRIC } \\
\text { MEAN }\end{array}$} & \multirow{2}{*}{$\begin{array}{l}\text { GEUME TRIC } \\
\text { DEVIATIDN }\end{array}$} \\
\hline & & & \\
\hline FE & PCF & 6.693222 & 1.81 \\
\hline MG & PCT & 1.835302 & 1.73 \\
\hline CA & PCT & 1.396456 & 1.52 \\
\hline TI & PCT & 0.456152 & 1.67 \\
\hline$M N$ & $P P M$ & 1501.268799 & 1.30 \\
\hline AG & $P P M$ & 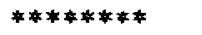 & 0 \\
\hline AS & PPM & ******** & 0 \\
\hline $\mathrm{AU}$ & PPM & $* * * * * * *$ & 0 \\
\hline B & PPM & 23.914093 & 2.13 \\
\hline BA & $P P M$ & 488.199219 & 2.50 \\
\hline $\mathrm{DE}$ & PPM & 0.644984 & 1.50 \\
\hline BI & PPM & $* * * * * * * *$ & $* * * * * *$ \\
\hline CD & PPM & $* * * * * * * *$ & $* * * * * *$ \\
\hline $\mathrm{CO}$ & $P P M$ & 19.642922 & 1.85 \\
\hline $\mathrm{CH}$ & $P P M$ & 60.219757 & 2.92 \\
\hline CU & PPM & 52.499969 & 2.76 \\
\hline L. A & PPM & 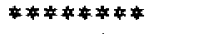 & $* * * * * *$ \\
\hline MŌ & PPM & 1.639061 & 3.49 \\
\hline$\therefore B$ & PPM & 4.994113 & 1.21 \\
\hline A [ & PPM & 33.852890 & 2.00 \\
\hline $\mathrm{PB}$ & PPM & 21.167540 & 2.16 \\
\hline
\end{tabular}

$\begin{array}{ll}\mathbf{H} & \mathrm{B} \\ 0 & 0 \\ 0 & 0 \\ 0 & 0 \\ 0 & 0 \\ 0 & 0 \\ 0 & 0 \\ 0 & 0 \\ 0 & 0 \\ 0 & 0 \\ 0 & 0 \\ 0 & 0 \\ 0 & 0 \\ 0 & 0 \\ 0 & 0 \\ 0 & 0 \\ 0 & 0 \\ 0 & 0 \\ 0 & 0 \\ 0 & 0 \\ 0 & 0 \\ 0 & 0 \\ 0 & 0 \\ 0 & 0 \\ 0 & 0 \\ 0 & 0 \\ 0 & 0 \\ 0 & 0 \\ 0 & 0 \\ 0 & 0 \\ 0 & 0 \\ 0 & 0 \\ & \end{array}$

REMARKS

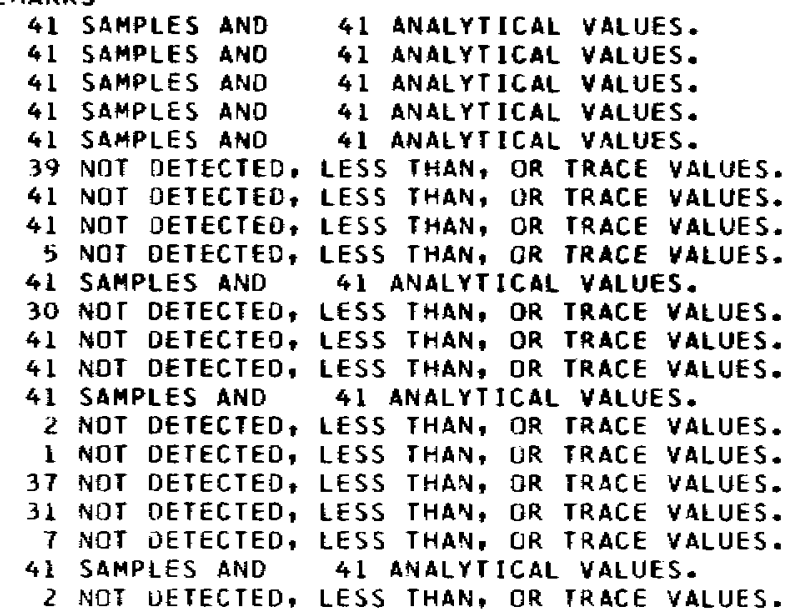

VALUES
VALYTCAL

41
41
41
41
41
2
0
0
36
41
11
0
0
41
39
40
4
10
34
41
39
0
39
11
41
41
0
41
7
41
2

2 REPOR TED VALUES. NO COMPUTATIONS. O REPORTED VALUES. NO COMPUTATIONS. 36 REPORTED VALUES.

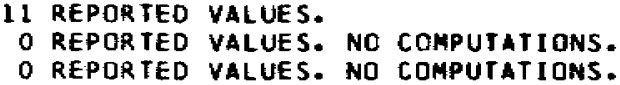




\begin{tabular}{|c|c|c|c|}
\hline 50 & PPin & $* * \div \div \div * *$ & 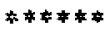 \\
\hline$S C$ & $P P M$ & 16.609333 & 1.82 \\
\hline Sid & PPA & 2.953623 & 5.24 \\
\hline $5 k$ & $\mathrm{PP}$ & 325.233154 & 1.33 \\
\hline$v$ & PPM & 199.653656 & 1.89 \\
\hline$w$ & $P P M$ & $* * * * * * * *$ & $* * * * * *$ \\
\hline$r$ & $P p, 4$ & 17.136931 & 1.35 \\
\hline$\angle Y$ & PPM & 115.247467 & 1.55 \\
\hline $2 R$ & $P P M$ & 74.556183 & 1.22 \\
\hline AU & PQM & 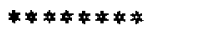 & 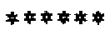 \\
\hline
\end{tabular}

41 NOT DETECTED, LESS THAN, OR IRACE VALUES. 2 NOT DETECTEO, LESS THAN, OR TRACE VALUES 30 NOT DETECTED, LESS THAN, OR TRACE VALUES. 41 SAMPLES ANU 41 ANALYTICAL VALUES.

41 SAMPLES AND 41 ANALYTICAL VALUES

41 NOT DETECTED, LESS THAN, DR TRACE VALUES. 41 SAMPLES AND, LESS THAN, DAR TRACE VAL

41 SAMPLES AND 41 ANALYTICAL VALUES.

34 NDT DEIECTED, LESS. THAN, OR TRACE VALUES.

41 SAMPLES AND 41 ANALYTICAL VALUES.

39 NOT DETECTED, LESS than, OR tRaCe Values.
O REPORTED VALUES. NO COMPUTATIONS. 39 REPURTEO VALUES.

11 REPORTED VALUES.

o Reported VAlues. no computations. 7 REPORTED VALUES.

2 REPUR TEO VALUES. NO COMPUTATIONS. 